\title{
Neutrino nonstandard interactions as a portal to test flavor symmetries
}

\author{
TseChun Wang ${ }^{*}$ and Ye-Ling Zhou ${ }^{\dagger}$ \\ Institute for Particle Physics Phenomenology, Department of Physics, Durham University, \\ Durham DH1 3LE, United Kingdom
}

(Received 9 August 2018; published 26 February 2019)

\begin{abstract}
Imposing non-Abelian discrete flavor symmetries on neutrino nonstandard interactions (NSIs) is discussed for the first time. For definiteness, we choose $A_{4}$ as the flavor symmetry, which is subsequently broken to the residual symmetry $Z_{2}$ in the neutrino sector. We provide a general discussion on the flavor structures of NSIs from higher-dimensional operators $(d \leq 8)$ without inducing unnecessary tree-level fourcharged-fermion interactions. Both $A_{4}$ - and $Z_{2}$-motivated NSI textures are obtained. UV completions of higher-dimensional operators lead to extra experimental constraints on NSI textures. We study the implementation of matter-effect NSIs in DUNE from a phenomenological point of view, and discover that DUNE can test $A_{4}$ with a high level of statistics. We also present the exclusion limits of sum rules suggested by UV-complete models. Our results show that the NSI effects, though predicted to be small for DUNE, could provide useful information that might extend our understanding of the flavor symmetry.
\end{abstract}

DOI: 10.1103/PhysRevD.99.035039

\section{INTRODUCTION}

Neutrino oscillation experiments have achieved great success in the last two decades [1-4]. Two neutrino mass-squared differences $\left(\Delta m_{21}^{2},\left|\Delta m_{31}^{2}\right|\right)$ and three mixing angles $\left(\theta_{12}, \theta_{23}, \theta_{13}\right)$ have been measured in the standard three-neutrino framework. Several next-generation oscillation experiments are proposed, such as the long-baseline (LBL) accelerator experiments DUNE [5] and T2HK [6], the intermediate-baseline reactor experiment JUNO [7,8], the SBN program [9], and the muon-decay experiments NuSTORM [10], MOMENT [11], and Neutrino Factory [12]. They are aimed at answering the remaining questions about neutrino oscillations: if $C P$ is violated in neutrino oscillations, what is the value of the Dirac-type $C P$-violating phase $\delta$, and which mass ordering $\left(\Delta m_{31}^{2}>0\right.$ or $\left.\Delta m_{31}^{2}<0\right)$ is true? In addition, the already known oscillation parameters can be measured to the percent level and the octant of $\theta_{23}$ $\left(\theta_{23}<45^{\circ}\right.$ or $\left.\theta_{23}>45^{\circ}\right)$ will be determined [13,14].

These experiments will also test the standard threeneutrino mixing scenario and might unveil new neutrino couplings beyond the Standard Model (SM). Neutrino nonstandard interactions (NSIs) provide a model-independent framework for studying new physics in neutrino oscillation experiments (for some reviews, see Ref. [15]). They are

\footnotetext{
tse-chun.wang@durham.ac.uk

†e-ling.zhou@durham.ac.uk
}

Published by the American Physical Society under the terms of the Creative Commons Attribution 4.0 International license. Further distribution of this work must maintain attribution to the author(s) and the published article's title, journal citation, and DOI. Funded by SCOAP . usually considered as effective descriptions of contributions from higher-dimensional operators mediated by heavy mediators [16-19], although they may also be induced by light mediators with very weak couplings (see, e.g., Refs. [20,21]). In neutrino oscillation experiments, NSIs may appear at neutrino sources, detectors, or during neutrino propagation. There are no experimental hints for NSIs at the source and the detector [15,22]. Current global-fit results for NSIs during neutrino propagation, i.e., matter-effect NSIs, have reached precisions from a few to tens of percent of the strength of the standard matter effect induced by the weak interaction [23]. Due to precision upgrades and because of non-negligible matter effects, the testability of NSIs in DUNE and T2HK (as well as its alternative, T2HKK) and the influences on measurements of mass ordering and $C P$ violation have received a lot of attention (see, e.g., Refs. [24-28]). For the study of NSIs in other future experiments, see, e.g., Refs. [29-33].

One important theoretical development promoted by neutrino oscillations is the application of flavor symmetries to understand lepton flavor mixing. It is directly triggered by the measured values of the mixing angles $\sin ^{2} \theta_{12} \sim 1 / 3$ and $\sin ^{2} \theta_{23} \sim 1 / 2$. In the framework of flavor symmetries, it is assumed that an underlying discrete flavor symmetry $G_{\mathrm{f}}$ that unifies the three flavors exists at some high energy scale. After the flavor symmetry is broken at a lower energy scale, special flavor structures arise. The most famous group used as a flavor symmetry is the tetrahedral group $A_{4}$ [34]. Most $A_{4}$ models naturally predict $\sin ^{2} \theta_{12}=1 / 3$ and $\sin ^{2} \theta_{23}=1 / 2$, but $\sin ^{2} \theta_{13}=0$ [35-37], i.e., the so-called tribimaximal (TBM) mixing [38]. One important feature of these models is the correspondence between the mixing and 
the existence of the residual symmetries $Z_{3}$ and $Z_{2}$ after $A_{4}$ breaking (for some reviews, see, e.g., Ref. [39]). $Z_{3}$ and $Z_{2}$ are subgroups of $A_{4}$. They are approximately preserved in the charged lepton and neutrino sectors, respectively, acting on charged leptons and neutrinos separately as

$$
\begin{aligned}
Z_{3}: e & \rightarrow e, \quad \mu \rightarrow e^{-i 2 \pi / 3} \mu, \quad \tau \rightarrow e^{i 2 \pi / 3} \tau \\
Z_{2}: \nu_{e} & \rightarrow \frac{1}{3}\left(-\nu_{e}+2 \nu_{\mu}+2 \nu_{\tau}\right), \quad \nu_{\mu} \rightarrow \frac{1}{3}\left(-\nu_{\mu}+2 \nu_{\tau}+2 \nu_{e}\right), \\
\nu_{\tau} & \rightarrow \frac{1}{3}\left(-\nu_{\tau}+2 \nu_{e}+2 \nu_{\mu}\right) .
\end{aligned}
$$

A slight breaking of the residual symmetries provides small corrections to the mixing, specifically generating a nonzero $\theta_{13}$ and making all mixing parameters compatible with oscillation data. The preferred parameters of these models will be tested by the future neutrino oscillation experiments.

Imposing flavor symmetries may not only influence the flavor mixing measured by neutrino oscillation experiments, but also contribute to other flavor-dependent phenomenological signatures, such as charged lepton flavor violation (CLFV). The influence of flavor symmetries on CLFV processes has been discussed in Refs. [40-46]. In particular, the essential contribution of $A_{4}$ and $Z_{3}$ to the CLFV decays of charged leptons have been carefully analyzed in Ref. [45]. The branching ratio sum rules of these processes were obtained therein, which can be regarded as specific features of flavor symmetries. In the neutrino sector, as the couplings are too weak, the phenomenological signatures of flavor symmetries beyond the standard neutrino oscillation measurements have been rarely discussed.

Previous discussions of NSIs in flavor symmetries have been limited to the Abelian case [20,21,47-49]. In these papers, by assuming a gauged $U(1)$ flavor symmetry, relatively sizable NSIs were generated via flavor-dependent gauge interactions mediated by a gauge boson with a mass around or below the GeV scale. Note that the $U(1)$ symmetries proposed in these works were not supposed to explain lepton flavor mixing. Thus, we do not expect any connection between NSIs and lepton flavor mixing.

In the non-Abelian case, $e, \mu$, and $\tau$ lepton doublets are arranged as a triplet in the flavor space, which both complicates the NSI construction and strengthens experimental constraints. However, if the non-Abelian discrete symmetry is a true symmetry, a combined study of the flavor symmetry and NSIs will be required in the future neutrino experiments. Regarding the $A_{4}$ case, the measurement of NSIs in neutrino oscillations provides an excellent opportunity to study the connection with $A_{4}$ and the residual symmetry $Z_{2}$ in the neutrino sector, as we will see later.

This work is aimed at discussing how to look for flavor symmetries and residual symmetries in the NSI measurements in neutrino oscillation experiments. We fix the flavor symmetry $A_{4}$ and residual symmetry $Z_{2}$ for definiteness. It is complementary to studies of $A_{4}$ and $Z_{3}$ in CLFV processes and in the standard neutrino oscillation measurements. By imposing the flavor symmetry in the fermion sectors, interesting NSI textures or sum rules of NSI parameters are obtained. Both NSIs from higherdimensional operators in the effective field theory (EFT) approach with respect to the electroweak symmetry and those mediated by specific beyond-the-SM particles will be discussed. The rest of this paper is organized as follows. We briefly review the TBM mixing realized in $A_{4}$ models in Sec. II. Section III is devoted to a systematic analysis of how to impose $A_{4}$ or $Z_{2}$ on higher-dimensional operators (with the dimension $d \leq 8$ ) which result in NSIs. A class of NSI textures based on $A_{4}$ and $Z_{2}$ are obtained. We only require that the three lepton doublets form a triplet of $A_{4}$; there are no requirements for the representations of other fermions in the flavor space. In Sec. IV we consider the UV completion of these operators. New particles in the UV sector impose additional experimental constraints on NSI parameters, and thus some textures are less constrained than others. We suggest that these textures have a priority to be discussed in the context of NSI measurements. In Sec. V, based on DUNE's experimental setup, we analyze the discovery potential of these textures. We summarize our paper in Sec. VI. In the main text of this paper, we focus on NSIs in matter. Connections between flavor symmetries and NSIs at the source and detector are strongly dependent upon the representations of the other fermions.

\section{FLAVOR SYMMETRIES AND RESIDUAL SYMMETRIES IN LEPTON MIXING}

We briefly review the realization of the TBM mixing in $A_{4}$ models and residual symmetries after $A_{4}$ is broken. $A_{4}$ is generated by two generators $\mathcal{S}$ and $\mathcal{T}$ with the requirements $\mathcal{S}^{2}=\mathcal{T}^{3}=(\mathcal{S T})^{3}=1$, and it contains 12 elements. It has four irreducible representations: three singlet representations $1, \mathbf{1}^{\prime}$, and $\mathbf{1}^{\prime \prime}$, and one triplet representation 3. The Kronecker products of two irreducible representations are reduced in the following way:

$$
\begin{array}{rlrl}
\mathbf{1} \times \mathbf{1}^{(1, \prime \prime)} & =\mathbf{1}^{(1, \prime \prime)}, & \mathbf{1}^{\prime} \times \mathbf{1}^{\prime}=\mathbf{1}^{\prime \prime}, \\
\mathbf{1}^{\prime \prime} \times \mathbf{1}^{\prime \prime} & =\mathbf{1}^{\prime}, & & \mathbf{1}^{\prime} \times \mathbf{1}^{\prime \prime}=\mathbf{1}, \\
\mathbf{3} \times \mathbf{1}^{(1, \prime \prime)} & =\mathbf{3}, & \mathbf{3} \times \mathbf{3}=\mathbf{1}+\mathbf{1}^{\prime}+\mathbf{1}^{\prime \prime}+\mathbf{3}_{\mathrm{S}}+\mathbf{3}_{\mathrm{A}},
\end{array}
$$

where the subscripts ${ }_{S}$ and ${ }_{A}$ stand for the symmetric and antisymmetric components, respectively.

We work in the Altarelli-Feruglio (AF) basis [36], where $\mathcal{T}$ and $\mathcal{S}$ are, respectively, given by

$$
\mathcal{T}=\left(\begin{array}{ccc}
1 & 0 & 0 \\
0 & \omega^{2} & 0 \\
0 & 0 & \omega
\end{array}\right), \quad \mathcal{S}=\frac{1}{3}\left(\begin{array}{ccc}
-1 & 2 & 2 \\
2 & -1 & 2 \\
2 & 2 & -1
\end{array}\right)
$$


This basis is widely used in the literature since the charged lepton mass matrix invariant under $\mathcal{T}$ is diagonal in this basis. The products of each two triplet representations $a=$ $\left(a_{1}, a_{2}, a_{3}\right)^{T}$ and $b=\left(b_{1}, b_{2}, b_{3}\right)^{T}$ can be expressed as

$$
\begin{aligned}
(a b)_{\mathbf{1}} & =a_{1} b_{1}+a_{2} b_{3}+a_{3} b_{2}, \\
(a b)_{\mathbf{1}^{\prime}} & =a_{3} b_{3}+a_{1} b_{2}+a_{2} b_{1}, \\
(a b)_{\mathbf{1}^{\prime \prime}} & =a_{2} b_{2}+a_{1} b_{3}+a_{3} b_{1}, \\
(a b)_{\mathbf{3}_{\mathrm{S}}} & =\frac{1}{2}\left(\begin{array}{l}
2 a_{1} b_{1}-a_{2} b_{3}-a_{3} b_{2} \\
2 a_{3} b_{3}-a_{1} b_{2}-a_{2} b_{1} \\
2 a_{2} b_{2}-a_{3} b_{1}-a_{1} b_{3}
\end{array}\right), \\
(a b)_{\mathbf{3}_{\mathrm{A}}} & =\frac{1}{2}\left(\begin{array}{l}
a_{2} b_{3}-a_{3} b_{2} \\
a_{1} b_{2}-a_{2} b_{1} \\
a_{3} b_{1}-a_{1} b_{3}
\end{array}\right) .
\end{aligned}
$$

The $A_{4}$ symmetry is broken at a certain lower scale. After the $A_{4}$ breaking, the residual symmetries $Z_{3}$ and $Z_{2}$ (which are generated by $\mathcal{T}$ and $\mathcal{S}$, respectively) are approximately preserved in the charged lepton and neutrino sectors, respectively. The residual symmetries constrain the lepton mass matrices and lead to the TBM mixing [38]. A sketch of how to realize the TBM mixing from $A_{4}$ is shown in Fig. 1.

The Lagrangian terms for generating charged lepton and neutrino masses are effectively realized by some higherdimensional operators. In the flavor space, the lepton doublets $L_{1}=\left(\nu_{e \mathrm{~L}}, e_{\mathrm{L}}\right), L_{2}=\left(\nu_{\mu \mathrm{L}}, \mu_{\mathrm{L}}\right)$, and $L_{3}=\left(\nu_{\tau \mathrm{L}}, \tau_{\mathrm{L}}\right)$ are often arranged as a triplet, $L \equiv\left(L_{1}, L_{2}, L_{3}\right)^{T}$. This arrangement holds for most flavor models with nonAbelian discrete symmetries, not just for $A_{4}$ models, in which the flavor symmetry contains a triplet irreducible

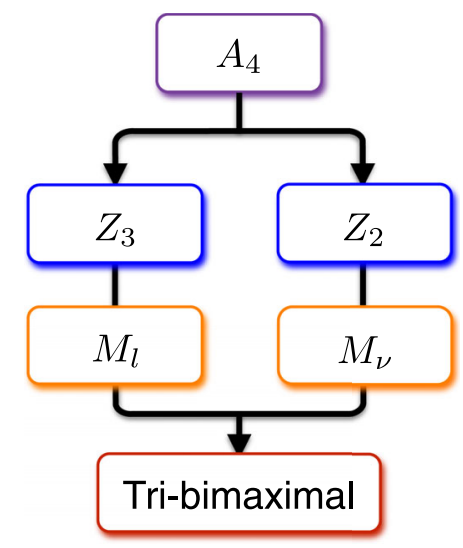

FIG. 1. A sketch of how the TBM mixing is generated in $A_{4}$ models. After $A_{4}$ is broken, residual symmetries $\left(Z_{3}\right.$ in the charged lepton sector and $Z_{2}$ in the neutrino sector) are preserved. These symmetries constrain the charged lepton and neutrino mass matrices, respectively, and finally result in the TBM mixing. The residual symmetries are just approximative symmetries in the model. Besides, there may be additional accidental symmetries in the model, which are not shown here. representation [39]. In $A_{4}$ models, the right-handed charged leptons $e_{R}, \mu_{R}$, and $\tau_{R}$ are often assigned as singlets $1, \mathbf{1}^{\prime}$, and $\mathbf{1}^{\prime \prime}$, respectively $[35,36]$. The relevant Lagrangian terms are effectively written as

$$
\begin{aligned}
-\mathcal{L}_{l}= & \frac{y_{e}}{\Lambda}(\bar{L} \varphi)_{\mathbf{1}} e_{R} H+\frac{y_{\mu}}{\Lambda}(\bar{L} \varphi)_{\mathbf{1}^{\prime \prime}} \mu_{R} H+\frac{y_{\tau}}{\Lambda}(\bar{L} \varphi)_{\mathbf{1}^{\prime}} \tau_{R} H \\
& + \text { H.c., } \\
-\mathcal{L}_{\nu}= & \frac{y_{1}}{2 \Lambda \Lambda_{\mathrm{W}}}\left(\left(\bar{L} \tilde{H} \tilde{H}^{T} L^{c}\right)_{\mathbf{3}_{S}} \chi\right)_{\mathbf{1}}+\frac{y_{2}}{2 \Lambda_{\mathrm{W}}}\left(\left(\bar{L} \tilde{H} \tilde{H}^{T} L^{c}\right)_{\mathbf{1}}\right. \\
& + \text { H.c., }
\end{aligned}
$$

where the Higgs $H \sim \mathbf{1}$ of $A_{4}$ and $\tilde{H}=i \sigma_{2} H^{*}$. We apply the dimension-five Weinberg operator $\left(\bar{L} \tilde{H} \tilde{H}^{T} L^{c}\right)$ to generate neutrino masses and $\Lambda_{\mathrm{W}}$ is the corresponding UV-complete scale. The operators in Eq. (5) involve flavons, denoted by $\varphi$ and $\chi$, and a new scale $\Lambda$ corresponding to the decoupling of some heavy $A_{4}$ multiplets.

Flavons play the key role in the flavor mixing. They gain vacuum expectation values (VEVs), leading to the breaking of the flavor symmetry and leaving residual symmetries in the charged lepton and neutrino sectors, respectively. The flavon VEVs $\varphi$ and $\chi$ preserving $Z_{3}$ and $Z_{2}$, respectively, ${ }^{1}$ i.e.,

$$
\mathcal{T} \varphi=\varphi, \quad \mathcal{S} \chi=\chi,
$$

take the following forms:

$$
\varphi=(1,0,0)^{T} v_{\varphi}, \quad \chi=(1,1,1)^{T} v_{\chi} .
$$

The resulting lepton mass matrices are represented as

$$
\begin{aligned}
M_{l} & =\left(\begin{array}{ccc}
y_{e} & 0 & 0 \\
0 & y_{\mu} & 0 \\
0 & 0 & y_{\tau}
\end{array}\right) \frac{v v_{\varphi}}{\sqrt{2} \Lambda}, \\
M_{\nu} & =\left(\begin{array}{ccc}
2 a+b & -a & -a \\
-a & 2 a & -a+b \\
-a & -a+b & 2 a
\end{array}\right),
\end{aligned}
$$

where $v=246 \mathrm{GeV}$ is the Higgs VEV, $a \equiv y_{1} v_{\chi} v^{2} /$ $\left(4 \Lambda \Lambda_{\mathrm{W}}\right)$, and $b \equiv y_{2} v^{2} /\left(2 \Lambda_{\mathrm{W}}\right)$. It is straightforward to check that the lepton mass matrices $M_{l}$ and $M_{\nu}$ satisfy $Z_{3}$ and $Z_{2}$, respectively,

$$
\mathcal{T} M_{l} M_{l}^{\dagger} \mathcal{T}^{\dagger}=M_{l} M_{l}^{\dagger}, \quad S M_{\nu} S^{T}=M_{\nu} .
$$

They are consistent with the residual symmetries satisfied by the flavon VEVs in Eq. (6). The charged lepton mass matrix $M_{l}$ is diagonal, and the neutrino mass matrix $M_{\nu}$ is diagonalized by the unitary matrix

\footnotetext{
${ }^{1}$ In the following, we do not specify the notation of flavons with flavon VEVs.
} 


$$
U_{\mathrm{TBM}}=\left(\begin{array}{ccc}
\frac{2}{\sqrt{6}} & \frac{1}{\sqrt{3}} & 0 \\
-\frac{1}{\sqrt{6}} & \frac{1}{\sqrt{3}} & \frac{1}{\sqrt{2}} \\
-\frac{1}{\sqrt{6}} & \frac{1}{\sqrt{3}} & -\frac{1}{\sqrt{2}}
\end{array}\right)
$$

and has eigenvalues $m_{1}=|3 a+b|, m_{2}=|b|$, and $m_{3}=$ $|3 a-b|$. The mixing matrix is identical to $U_{\mathrm{TBM}}$. This is the so-called the TBM mixing pattern, from which we obtain $\sin \theta_{13}=0, \sin \theta_{12}=1 / \sqrt{3}$, and $\sin \theta_{23}=1 / \sqrt{2}$. Figure 1 presents a sketch of how the TBM mixing is generated in $A_{4}$ models.

The TBM mixing should only be considered as a leadingorder result since it is not consistent with neutrino oscillation data. Deviations from the TBM mixing have to be included in flavor model construction. The deviations are usually obtained from certain subleading interactions which break the $Z_{3}$ or $Z_{2}$ residual symmetries. It is crucial to obtain suitable deviations that are all compatible with current data. (For very recent $A_{4}$ models consistent with current oscillation data, see, e.g., Refs. [50,51] and references therein.) These deviations may contribute to NSIs as subleading effects. However, there are various successful flavor models, and the deviations are usually model dependent. In addition, these subleading effects are negligible in current NSI measurements. Therefore, we will not consider small corrections to NSIs resulted from small deviations from the TBM mixing.

\section{NSI TEXTURES PREDICTED BY FLAVOR SYMMETRIES IN EFT}

In neutrino oscillation experiments, NSIs may appear in processes of neutrino production at the source, propagation in matter and detection at the detector. The matter-effect NSIs are customarily described by a $3 \times 3$ Hermitian matrix $\epsilon$ added to an effective Hamiltonian $H$ in the flavor basis,

$$
\begin{aligned}
H= & \frac{1}{2 E}\left\{U\left(\begin{array}{ccc}
0 & 0 & 0 \\
0 & \Delta m_{21}^{2} & 0 \\
0 & 0 & \Delta m_{31}^{2}
\end{array}\right) U^{\dagger}+A\left(\begin{array}{lll}
1 & 0 & 0 \\
0 & 0 & 0 \\
0 & 0 & 0
\end{array}\right)\right. \\
& \left.+A\left(\begin{array}{ccc}
\epsilon_{e e} & \epsilon_{e \mu} & \epsilon_{e \tau} \\
\epsilon_{\mu e} & \epsilon_{\mu \mu} & \epsilon_{\mu \tau} \\
\epsilon_{\tau e} & \epsilon_{\tau \mu} & \epsilon_{\tau \tau}
\end{array}\right)\right\}
\end{aligned}
$$

where $\epsilon_{\alpha \beta}=\epsilon_{\beta \alpha}^{*}$ holds, and $A=2 \sqrt{2} G_{F} N_{e} E$ is the usual matter effect where $N_{e}$ is the electron number density in the Earth and $E$ is the neutrino beam energy. The effective Hamiltonian for antineutrino oscillation is obtained after the replacements $U \rightarrow U^{*}, A \rightarrow-A$ and $\varepsilon_{\alpha \beta} \rightarrow \varepsilon_{\alpha \beta}^{*}$. In this section, by assuming NSIs obtained from higherdimensional operators, we embed $A_{4}$ or its residual symmetry $Z_{2}$ into these operators and systematically analyze how to obtain NSI textures from the symmetry.

\section{A. NSIs from higher-dimensional operators}

We assume that NSIs arise from effective higher-dimensional operators and these operators satisfy the following conditions:

(1) Lorentz invariance and the SM gauge symmetry $S U(2)_{\mathrm{L}} \times U(1)_{\mathrm{Y}}$ around or above the electroweak scale are required.

(2) Since neutrino oscillation experiments cannot test lepton-number-violating (LNV) or baryon-numberviolating processes, we select lepton- and baryonnumber-conserving operators. ${ }^{2}$

(3) We only focus on operators with four fermions. The simplest operators have dimension $d=6$, and the operators with $d>6$ consist of four fermions and $d-6$ Higgs fields. ${ }^{3}$ In the following, we briefly denote the remaining SM fermion contents as

$$
\begin{array}{ll}
E_{\mathrm{R}}=\left(e_{\mathrm{R}}, \mu_{\mathrm{R}}, \tau_{\mathrm{R}}\right)^{T}, & U_{\mathrm{R}}=\left(u_{\mathrm{R}}, c_{\mathrm{R}}, t_{\mathrm{R}}\right)^{T}, \\
D_{\mathrm{R}}=\left(d_{\mathrm{R}}, s_{\mathrm{R}}, b_{\mathrm{R}}\right)^{T}, & Q=\left(Q_{1}, Q_{2}, Q_{3}\right)^{T},
\end{array}
$$

where $Q_{1}=\left(u_{\mathrm{L}}, d_{\mathrm{L}}\right), Q_{2}=\left(c_{\mathrm{L}}, s_{\mathrm{L}}\right), Q_{3}=\left(t_{\mathrm{L}}, b_{\mathrm{L}}\right)$.

(4) For neutrinos propagating in matter, at least two $L$ 's must be involved in the relevant operators. As a comparison, operators for neutrino production and detection involve at least one $L$.

(5) Furthermore, we impose one more requirement: we only consider NSIs that avoid the strong constraints from four-charged-fermion interactions, e.g., rare lepton-flavor-violating decays of leptons and hadrons. Since left-handed charged leptons and neutrinos belong to the same electroweak doublet in the SM, any NSI effects from higher-dimensional operators are related to an interaction involving at least one charged lepton. Once all final and initial states of the latter interaction are electrically charged fermions, i.e., charged leptons and quarks, the operator and the relevant NSI parameters should have been strongly constrained by these "visible" processes. For example, the nonstandard $\nu_{\mu}+(e, u, d) \rightarrow \nu_{e}+$ $(e, u, d)$ propagation in matter may be constrained by $\mu+(e, u, d) \rightarrow e+(e, u, d)$ in CLFV measurements.

The following classes of operators and their conjugates are allowed by the first four requirements:

$$
\begin{gathered}
\bar{L} E_{\mathrm{R}} \overline{D_{\mathrm{R}}} Q, \quad \bar{L} E_{\mathrm{R}} \bar{Q} U_{\mathrm{R}}, \quad \bar{L} L \bar{F} F, \quad \text { with } \quad F=L, \\
E_{\mathrm{R}}, \quad Q, \quad U_{\mathrm{R}}, \quad D_{\mathrm{R}}
\end{gathered}
$$

\footnotetext{
${ }^{2}$ This does not mean that the lepton number or baryon number cannot be broken at the UV-complete scale, as will be discussed in the next section.

${ }^{3}$ Operators modifying neutrino kinetic terms may also contribute to the NSIs through the nondiagonal $Z$ mediation. These effects are small $\left(\lesssim 10^{-3}\right)$ due to the nonunitarity of the PMNS matrix $[28,52]$, and will not be considered here.
} 
for $d=6$ and

$$
\begin{array}{lrl}
\bar{L} L \overline{D_{\mathrm{R}}} U_{\mathrm{R}} H^{*} H^{*}, & \bar{L} E_{\mathrm{R}} \overline{U_{\mathrm{R}}} Q H H, & \bar{L} E_{\mathrm{R}} \bar{Q} D_{\mathrm{R}} H H, \quad \bar{L} E_{\mathrm{R}} \bar{L} E_{\mathrm{R}} H H, \\
\bar{L} E_{\mathrm{R}} \overline{D_{\mathrm{R}}} Q H^{*} H, & \bar{L} E_{\mathrm{R}} \bar{Q} U_{\mathrm{R}} H^{*} H, & \bar{L} L \bar{F} F H^{*} H, \quad \text { with } \quad F=L, \quad E_{\mathrm{R}}, \quad Q, \quad U_{\mathrm{R}}, \quad D_{\mathrm{R}}
\end{array}
$$

for $d=8$. Here we have not written out the necessary $\Gamma$ matrices, gauge indices, and flavor indices. Lepton and baryon number conservation forbids any dimension-seven operators involving four fermions. After the Higgs acquires a VEV $\langle H\rangle=(0,1)^{T}\left(2 \sqrt{2} G_{F}\right)^{-1 / 2}$, these operators can be classified into two types: those that preserves electroweak symmetry and those that do not. Taking the last requirement into account, we extract the following operators:

(1) The first class is explicitly given by

$$
\varepsilon_{a c} \varepsilon_{b d}\left(\overline{L_{a \alpha}} \gamma^{\mu} L_{b \beta}\right)\left(\overline{L_{c \gamma}} \gamma_{\mu} L_{d \delta}\right), \quad \varepsilon_{a c} \varepsilon_{b d}\left(\overline{L_{a \alpha}} \gamma^{\mu} L_{b \beta}\right)\left(\overline{L_{c \gamma}} \gamma_{\mu} L_{d \delta}\right) H^{\dagger} H,
$$

where $\alpha, \beta, \gamma, \delta=1,2,3$ are flavor indices, $a, b, c, d=1,2$ are $S U(2)_{\mathrm{L}}$ doublet indices, and nonvanishing entries of $\varepsilon_{a b}$ are given by $\varepsilon_{12}=-\varepsilon_{21}=1$. Specifically, we denote the flavor indices in the lepton sector as $(1,2,3)=(e, \mu, \tau)$. Using the relation $\varepsilon_{a c} \varepsilon_{c d}=\delta_{a b} \delta_{c d}-\delta_{a d} \delta_{b c}$ and the Fierz identity, we expand the first term of the above equation and obtain $\left(\overline{L_{a \alpha}} \gamma^{\mu} L_{a \beta}\right)\left(\overline{L_{c \gamma}} \gamma_{\mu} L_{c \delta}\right)-\left(\overline{L_{a \alpha}} \gamma^{\mu} L_{a \delta}\right)\left(\overline{L_{c \gamma}} \gamma_{\mu} L_{c \beta}\right)$, i.e.,

$$
\left(\overline{\nu_{\alpha \mathrm{L}}} \gamma^{\mu} \nu_{\beta \mathrm{L}}\right)\left(\overline{E_{\gamma \mathrm{L}}} \gamma_{\mu} E_{\delta \mathrm{L}}\right)+\left(\overline{\gamma_{\gamma \mathrm{L}}} \gamma^{\mu} \nu_{\delta \mathrm{L}}\right)\left(\overline{E_{\alpha \mathrm{L}}} \gamma_{\mu} E_{\beta \mathrm{L}}\right)-\left(\overline{\nu_{\alpha \mathrm{L}}} \gamma^{\mu} \nu_{\delta \mathrm{L}}\right)\left(\overline{E_{\gamma \mathrm{L}}} \gamma_{\mu} E_{\beta \mathrm{L}}\right)-\left(\overline{\nu_{\gamma \mathrm{L}}} \gamma^{\mu} \nu_{\beta \mathrm{L}}\right)\left(\overline{E_{\alpha \mathrm{L}}} \gamma_{\mu} E_{\delta \mathrm{L}}\right)
$$

which we denote as $\mathcal{O}_{\alpha \beta \gamma \delta}^{1}$. Note that $\mathcal{O}_{\alpha \beta \gamma \delta}^{1}=-\mathcal{O}_{\gamma \beta \alpha \delta}^{1}=-\mathcal{O}_{\alpha \delta \gamma \beta}^{1}=\mathcal{O}_{\gamma \delta \alpha \beta}^{1}$ is satisfied. This term can lead to NSIs of neutrinos interacting with electrons $\left(\nu_{\alpha} e \rightarrow \nu_{\beta} e\right)$ during neutrino propagation, but it has no influence on four-chargedlepton interactions, such as the scattering $\mu e \rightarrow e e$ or the rare decay $\mu \rightarrow e e e$, and thus are not directly constrained by the latter. The second term in Eq. (15) gives the same information as $\mathcal{O}_{\alpha \beta \gamma \delta}^{1}$, and thus it is not necessary to consider them separately.

(2) The second class of operators are

$$
\begin{array}{lcc}
\left(\overline{L_{\alpha}} \tilde{H} \gamma^{\mu} \tilde{H}^{\dagger} L_{\beta}\right)\left(\overline{U_{\gamma \mathrm{R}}} \gamma_{\mu} U_{\delta \mathrm{R}}\right), & \left(\overline{L_{\alpha}} \tilde{H} \gamma^{\mu} \tilde{H}^{\dagger} L_{\beta}\right)\left(\overline{D_{\gamma \mathrm{R}}} \gamma_{\mu} D_{\delta \mathrm{R}}\right), & \left(\overline{L_{\alpha}} \tilde{H} \gamma^{\mu} \tilde{H}^{\dagger} L_{\beta}\right)\left(\overline{E_{\gamma \mathrm{R}}} \gamma_{\mu} E_{\delta \mathrm{R}}\right), \\
\left(\overline{L_{\alpha}} \tilde{H} \gamma^{\mu} \tilde{H}^{\dagger} L_{\beta}\right)\left(\overline{Q_{\gamma}} \gamma_{\mu} Q_{\delta}\right), & \left(\overline{L_{\alpha}} \tilde{H} \gamma^{\mu} \tilde{H}^{\dagger} L_{\beta}\right)\left(\overline{L_{\gamma}} \gamma_{\mu} L_{\delta}\right), \\
\left(\overline{L_{\alpha}} \tilde{H} \gamma^{\mu} L_{b \beta}\right)\left(\overline{Q_{b \gamma}} \gamma_{\mu} \tilde{H}^{\dagger} Q_{\delta}\right), & \varepsilon_{b c}\left(\overline{L_{\alpha}} \tilde{H} \gamma^{\mu} L_{b \beta}\right)\left(\overline{Q_{\gamma}} H \gamma_{\mu} Q_{c \delta}\right), \\
\left(\overline{L_{\alpha}} \tilde{H} \gamma^{\mu} H^{\dagger} L_{\beta}\right)\left(\overline{D_{\gamma \mathrm{R}}} \gamma_{\mu} U_{\delta \mathrm{R}}\right), & \left(\overline{L_{\alpha}} \tilde{H} \sigma^{\mu \nu} E_{\beta \mathrm{R}}\right)\left(\overline{Q_{\gamma}} H \sigma_{\mu \nu} U_{\delta \mathrm{R}}\right), \\
\left(\overline{L_{\alpha}} \tilde{H} E_{\beta \mathrm{R}}\right)\left(\overline{D_{\gamma \mathrm{R}}} \tilde{H}^{\dagger} Q_{\delta}\right), & \left(\overline{L_{\alpha}} \tilde{H} E_{\beta \mathrm{R}}\right)\left(\overline{Q_{\gamma}} H U_{\delta \mathrm{R}}\right) .
\end{array}
$$

After the Higgs acquires a VEV, the above operators are effectively reduced to 11 four-fermion interactions:

$$
\begin{aligned}
& \left(\overline{\nu_{\alpha \mathrm{L}}} \gamma^{\mu} \nu_{\beta \mathrm{L}}\right)\left(\overline{U_{\gamma \mathrm{R}}} \gamma_{\mu} U_{\delta \mathrm{R}}\right), \quad\left(\overline{\nu_{\alpha \mathrm{L}}} \gamma^{\mu} \nu_{\beta \mathrm{L}}\right)\left(\overline{D_{\gamma \mathrm{R}}} \gamma_{\mu} D_{\delta \mathrm{R}}\right), \quad\left(\overline{\nu_{\alpha \mathrm{L}}} \gamma^{\mu} \nu_{\beta \mathrm{L}}\right)\left(\overline{E_{\gamma \mathrm{R}}} \gamma_{\mu} E_{\delta \mathrm{R}}\right), \\
& \left(\overline{\nu_{\alpha \mathrm{L}}} \gamma^{\mu} \nu_{\beta \mathrm{L}}\right)\left(\overline{U_{\gamma \mathrm{L}}} \gamma_{\mu} U_{\delta \mathrm{L}}+\overline{D_{\gamma \mathrm{L}}} \gamma_{\mu} D_{\delta \mathrm{L}}\right), \quad\left(\overline{\nu_{\alpha \mathrm{L}}} \gamma^{\mu} \nu_{\beta \mathrm{L}}\right)\left(\overline{\nu_{\gamma \mathrm{L}}} \gamma_{\mu} \nu_{\delta \mathrm{L}}+\overline{E_{\gamma \mathrm{L}}} \gamma_{\mu} E_{\delta \mathrm{L}}\right), \\
& \left(\overline{\nu_{\alpha \mathrm{L}}} \gamma^{\mu} \nu_{\beta \mathrm{L}}\right)\left(\overline{U_{\gamma \mathrm{L}}} \gamma_{\mu} U_{\delta \mathrm{L}}\right)+\left(\overline{\nu_{\alpha \mathrm{L}}} \gamma^{\mu} E_{\beta \mathrm{L}}\right)\left(\overline{D_{\gamma \mathrm{L}}} \gamma_{\mu} U_{\delta \mathrm{L}}\right), \quad\left(\overline{\nu_{\alpha \mathrm{L}}} \gamma^{\mu} \nu_{\beta \mathrm{L}}\right)\left(\overline{D_{\gamma \mathrm{L}}} \gamma_{\mu} D_{\delta \mathrm{L}}\right)-\left(\overline{\nu_{\alpha \mathrm{L}}} \gamma^{\mu} E_{\beta \mathrm{L}}\right)\left(\overline{D_{\gamma \mathrm{L}}} \gamma_{\mu} U_{\delta \mathrm{L}}\right), \\
& \left(\overline{\nu_{\alpha \mathrm{L}}} \gamma^{\mu} E_{\beta \mathrm{L}}\right)\left(\overline{D_{\gamma \mathrm{R}}} \gamma_{\mu} U_{\delta \mathrm{R}}\right), \quad\left(\overline{\nu_{\alpha \mathrm{L}}} \sigma^{\mu \nu} E_{\beta \mathrm{R}}\right)\left(\overline{D_{\gamma \mathrm{L}}} \sigma_{\mu \nu} U_{\delta \mathrm{R}}\right), \\
& \left(\overline{\nu_{\alpha \mathrm{L}}} E_{\beta \mathrm{R}}\right)\left(\overline{D_{\gamma \mathrm{R}}} U_{\delta \mathrm{L}}\right), \quad\left(\overline{\nu_{\alpha \mathrm{L}}} E_{\beta \mathrm{R}}\right)\left(\overline{D_{\gamma \mathrm{L}}} U_{\delta \mathrm{R}}\right) .
\end{aligned}
$$

In the above operators, the first five terms, denoted by $\mathcal{O}_{\alpha \beta \gamma \delta}^{2,3,4,5,6}$, respectively, contribute to NSIs in matter during neutrino propagation. The next two terms, denoted by $\mathcal{O}_{\alpha \beta \gamma \delta}^{7,8}$, respectively, contribute to and correlate between NSIs at the neutrino source and detector and NSIs for neutrino mediation in matter. The final four terms, denoted by $\mathcal{O}_{\alpha \beta \gamma \delta}^{9,10,11,12}$, respectively, contribute to NSIs in the neutrino production and detection processes. For more discussions on textures of NSIs in these processes, please see Appendix B.

The effective operators describing neutrino NSIs for neutrino propagation can be expressed as

$$
\mathcal{L}_{\mathrm{NSI}}=2 \sqrt{2} G_{F} \sum_{p=1}^{8} c_{\alpha \beta \gamma \delta}^{p} \mathcal{O}_{\alpha \beta \gamma \delta}^{p}+\text { H.c. }
$$


where two same-flavor indices should be summed. The operators in Eqs. (16) and (18) form a full list of NSI operators with $d \leq 8$ before electroweak symmetry breaking. We have checked that all of the other NSIs with $d \leq 8$ operators can be represented as a linear combination of these $\mathcal{O}_{\alpha \beta \gamma \delta}^{p}$. Matching with the effective NSI matrix $\epsilon$ in Eq. (11), we obtain

$$
\epsilon_{\alpha \beta}=\epsilon_{\alpha \beta}^{e}+\left(2+\frac{N_{n}}{N_{e}}\right) \epsilon_{\alpha \beta}^{u}+\left(1+2 \frac{N_{n}}{N_{e}}\right) \epsilon_{\alpha \beta}^{d},
$$

where $N_{n}$ is the neutron number density and

$$
\begin{aligned}
& \epsilon_{\alpha \beta}^{e}=c_{\alpha \beta 11}^{1}+c_{\alpha \beta 11}^{4}+c_{\alpha \beta 11}^{6}, \\
& \epsilon_{\alpha \beta}^{u}=c_{\alpha \beta 11}^{2}+c_{\alpha \beta 11}^{5}+c_{\alpha \beta 11}^{7}, \\
& \epsilon_{\alpha \beta}^{d}=c_{\alpha \beta 11}^{3}+c_{\alpha \beta 11}^{5}+c_{\alpha \beta 11}^{8} .
\end{aligned}
$$

For $\mathcal{O}_{\alpha \beta \gamma \delta}^{1}$, it is easy to confirm that $c_{\alpha \beta \gamma \delta}^{1}=-c_{\gamma \beta \alpha \delta}^{1}=c_{\alpha \delta \gamma \beta}^{1}$, and thus $c_{e \beta 11}^{1}$ and $c_{\alpha e 11}^{1}$ always vanish. Therefore, $\mathcal{O}_{\alpha \beta \gamma \delta}^{1}$ will not contribute to the first column or first row of $\epsilon$.

\section{B. NSI textures predicted by $\boldsymbol{A}_{4}$}

We consider how neutrino NSIs from the higherdimensional operators are constrained by $A_{4}$. We require that the higher-dimensional operators are invariant under the symmetry $A_{4}$ and consider which kinds of NSI textures we could gain from the symmetry. As we only care about matter-effect NSI textures, we limit our discussion to the operators $\mathcal{O}^{1-8}$. In Appendix B, we list the NSI textures at the source and detector from the operators $\mathcal{O}^{7-12}$.

We follow Sec. II in which the lepton doublets $L=$ $\left(L_{1}, L_{2}, L_{3}\right)^{T}$ are often arranged as a triplet 3 of $A_{4}{ }^{4}$ Besides, we do not specify the representations for the other fermions in the flavor space. In other words, the righthanded charged leptons, left-handed quarks, and righthanded quarks could be any irreducible representations of $A_{4}, \mathbf{1}, \mathbf{1}^{\prime}, \mathbf{1}^{\prime \prime}$, or 3 . It is worth noting that we do not specify whether $A_{4}$ can be responsible for the quark mixing in this work. If all quarks are arranged as the singlet representation 1, quark flavor mixing is totally independent of $A_{4}$. We scan for all of these possibilities, and find the following NSI textures:

$\mathbb{T}_{11} \equiv \mathbb{1}=\left(\begin{array}{ccc}1 & 0 & 0 \\ 0 & 1 & 0 \\ 0 & 0 & 1\end{array}\right), \quad \mathbb{T}_{12}=\left(\begin{array}{ccc}2 & 0 & 0 \\ 0 & -1 & 0 \\ 0 & 0 & -1\end{array}\right)$,

$\mathbb{T}_{13}=\left(\begin{array}{ccc}0 & 0 & 0 \\ 0 & 1 & 0 \\ 0 & 0 & -1\end{array}\right)$.

\footnotetext{
${ }^{4}$ In the AF basis, the conjugate of $L$ should be arranged as $\bar{L}=\left(\overline{L_{1}}, \overline{L_{3}}, \overline{L_{2}}\right)^{T}$.
}

In the following, we explain how to get these textures.

The first operator $c_{\alpha \beta \gamma \delta}^{1} \mathcal{O}_{\alpha \beta \gamma \delta}^{1}$, i.e., the dimension-six $\varepsilon_{a c} \varepsilon_{b d} c_{\alpha \beta \gamma \delta}^{1}\left(\overline{L_{a \alpha}} \gamma^{\mu} L_{b \beta}\right)\left(\overline{L_{c \gamma}} \gamma_{\mu} L_{d \delta}\right)$, satisfies the antipermutation property of two $L$ 's and two $\bar{L}$ 's, as shown in Eq. (16), which results in $c_{e \beta 11}^{1}=c_{\alpha e 11}^{1}=0$. There are five independent $A_{4}$-invariant operators:

$$
\begin{aligned}
& (\bar{L} L)_{\mathbf{1}}(\bar{L} L)_{\mathbf{1}}, \quad(\bar{L} L)_{\mathbf{1}^{\prime}}(\bar{L} L)_{\mathbf{1}^{\prime \prime}}, \quad(\bar{L} L)_{\mathbf{3}_{\mathrm{S}}}(\bar{L} L)_{\mathbf{3}_{\mathrm{s}}}, \\
& (\bar{L} L)_{\mathbf{3}_{\mathrm{A}}}(\bar{L} L)_{\mathbf{3}_{\mathrm{A}}}, \quad(\bar{L} L)_{\mathbf{3}_{\mathrm{S}}}(\bar{L} L)_{\mathbf{3}_{\mathrm{A}}} .
\end{aligned}
$$

Here we have ignored the unnecessary flavor-independent notations, including the $S U(2)_{\mathrm{L}}$ indices, $\Gamma$ matrices, and the Higgs field. The subscripts are the same as in Eq. (4). Taking account of the Clebsch-Goldan (CG) coefficients in Eq. (4), we obtain

$$
c_{\mu \mu 11}^{1}=c_{\tau \tau 11}^{1}, \quad c_{e e 11}^{1}=c_{\alpha \beta 11}^{1}=0 \quad \text { for } \alpha \neq \beta
$$

for the first four operators, which lead to the NSI texture

$$
\mathbb{T}_{12}^{\prime} \equiv\left(\begin{array}{ccc}
0 & 0 & 0 \\
0 & 1 & 0 \\
0 & 0 & 1
\end{array}\right) \propto 2 \mathbb{T}_{11}-\mathbb{T}_{12}
$$

The last operator gives a vanishing $c_{\alpha \beta 11}^{1}$ and thus does not contribute to NSIs.

For the second entry in Table I, $c_{\alpha \beta \gamma \delta}^{2} \mathcal{O}_{\alpha \beta \gamma \delta}^{2}$, i.e., the dimension-eight $\left(\overline{L_{\alpha}} \tilde{H} \gamma^{\mu} \tilde{H}^{\dagger} L_{\beta}\right)\left(\overline{U_{\gamma \mathrm{R}}} \gamma_{\mu} U_{\delta \mathrm{R}}\right)$, the $A_{4}$ invariant operators depend on the flavor representation of $U_{\mathrm{R}}$ :

(1) If $U_{1 \mathrm{R}}$ is arranged as a singlet $\mathbf{1}^{(1, \prime)}$ of $A_{4}$, there is only one $A_{4}$-invariant operator:

$$
(\bar{L} L)_{\mathbf{1}}\left(\overline{U_{1 \mathrm{R}}} U_{1 \mathrm{R}}\right)_{\mathbf{1}}
$$

It leads to the following relations of the coefficients:

$$
c_{e e 11}^{2}=c_{\mu \mu 11}^{2}=c_{\tau \tau 11}^{2}, \quad c_{\alpha \beta 11}^{2}=0 \text { for } \alpha \neq \beta .
$$

Representations of $U_{2 \mathrm{R}}$ and $U_{3 \mathrm{R}}$ are irrelevant for our discussion since $U_{2 \mathrm{R}}$ and $U_{3 \mathrm{R}}$ do not contribute to the low-energy NSIs.

(2) If $U_{\mathrm{R}}=\left(U_{1 \mathrm{R}}, U_{2 \mathrm{R}}, U_{3 \mathrm{R}}\right)^{T}$ is a triplet $\mathbf{3}$ of $A_{4}$, there are seven independent $A_{4}$-invariant operators:

$$
\begin{aligned}
& (\bar{L} L)_{\mathbf{1}}\left(\overline{U_{\mathrm{R}}} U_{\mathrm{R}}\right)_{\mathbf{1}},(\bar{L} L)_{\mathbf{1}^{\prime}}\left(\overline{U_{\mathrm{R}}} U_{\mathrm{R}}\right)_{\mathbf{1}^{\prime \prime}},(\bar{L} L)_{\mathbf{1}^{\prime \prime}}\left(\overline{U_{\mathrm{R}}} U_{\mathrm{R}}\right)_{\mathbf{1}^{\prime}}, \\
& (\bar{L} L)_{\mathbf{3}_{\mathrm{S}}}\left(\overline{U_{\mathrm{R}}} U_{\mathrm{R}}\right)_{\mathbf{3}_{\mathrm{S}}},(\bar{L} L)_{\mathbf{3}_{\mathrm{A}}}\left(\overline{U_{\mathrm{R}}} U_{\mathrm{R}}\right)_{\mathbf{3}_{\mathrm{S}}}, \\
& (\bar{L} L)_{\mathbf{3}_{\mathrm{S}}}\left(\overline{U_{\mathrm{R}}} U_{\mathrm{R}}\right)_{\mathbf{3}_{\mathrm{A}}},(\bar{L} L)_{\mathbf{3}_{\mathrm{A}}}\left(\overline{U_{\mathrm{R}}} U_{\mathrm{R}}\right)_{\mathbf{3}_{\mathrm{A}}} .
\end{aligned}
$$

The first operator gives the same correlation as in Eq. (27), while $(\bar{L} L)_{\mathbf{3}_{\mathrm{S}}}\left(\overline{U_{\mathrm{R}}} U_{\mathrm{R}}\right)_{\mathbf{3}_{\mathrm{S}}}$ and $(\bar{L} L)_{\mathbf{3}_{\mathrm{A}}}\left(\overline{U_{\mathrm{R}}} U_{\mathrm{R}}\right)_{\mathbf{3}_{\mathrm{S}}}$ give rise to 
TABLE I. Higher-dimensional operators $(d \leq 8)$ that may contribute to NSIs in neutrino oscillation experiments. $\mathrm{S}, \mathrm{M}$, and D represent NSIs at a source, in matter, and at a detector, respectively.

\begin{tabular}{|c|c|c|c|}
\hline Label & Before EW breaking & After EW breaking & Observation \\
\hline $\mathcal{O}^{1}$ & $\begin{array}{l}\varepsilon_{a c} \varepsilon_{b d}\left(L_{a \alpha}^{-} \gamma^{\mu} L_{b \beta}\right)\left(L_{c \gamma}^{-} \gamma_{\mu} L_{d \delta}\right), \\
\varepsilon_{a c} \varepsilon_{b d}\left(L_{a \alpha}^{-} \gamma^{\mu} L_{b \beta}\right)\left(L_{c \gamma}^{-} \gamma_{\mu} L_{d \delta}\right) H^{\dagger} H\end{array}$ & 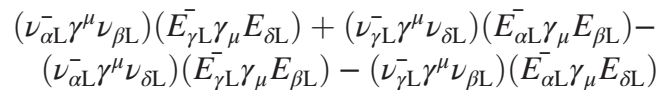 & M \\
\hline $\mathcal{O}^{2}$ & $\left(\overline{L_{\alpha}} \tilde{H} \gamma^{\mu} \tilde{H}^{\dagger} L_{\beta}\right)\left(\overline{U_{\gamma \mathrm{R}}} \gamma_{\mu} U_{\delta \mathrm{R}}\right)$ & $\left(\nu_{\alpha \mathrm{L}}^{-} \gamma^{\mu} \nu_{\beta \mathrm{L}}\right)\left(U_{\gamma \mathrm{R}} \gamma_{\mu} U_{\delta \mathrm{R}}\right)$ & M \\
\hline $\mathcal{O}^{3}$ & $\left(\overline{L_{\alpha}} \tilde{H} \gamma^{\mu} \tilde{H}^{\dagger} L_{\beta}\right)\left(\overline{D_{\gamma \mathrm{R}}} \gamma_{\mu} D_{\delta \mathrm{R}}\right)$ & $\left(\nu_{\alpha \mathrm{L}}^{-} \gamma^{\mu} \nu_{\beta \mathrm{L}}\right)\left(D_{\gamma \mathrm{R}}^{-} \gamma_{\mu} D_{\delta \mathrm{R}}\right)$ & $\mathrm{M}$ \\
\hline $\mathcal{O}^{4}$ & $\left(\overline{L_{\alpha}} \tilde{H} \gamma^{\mu} \tilde{H}^{\dagger} L_{\beta}\right)\left(\overline{E_{\gamma \mathrm{R}}} \gamma_{\mu} E_{\delta \mathrm{R}}\right)$ & $\left(\nu_{\alpha \mathrm{L}}^{-} \gamma^{\mu} \nu_{\beta \mathrm{L}}\right)\left(E_{\gamma \mathrm{R}}^{-} \gamma_{\mu} E_{\delta \mathrm{R}}\right)$ & M \\
\hline $\mathcal{O}^{5}$ & $\left({\overline{L_{\alpha}}}_{\tilde{H}} \gamma^{\mu} \tilde{H}^{\dagger} L_{\beta}\right)\left(\bar{Q}_{\gamma} \gamma_{\mu} Q_{\delta}\right)$ & $\left(\nu_{\alpha \mathrm{L}}^{-} \gamma^{\mu} \nu_{\beta \mathrm{L}}\right)\left(\overline{U_{\gamma \mathrm{L}}} \gamma_{\mu} U_{\delta \mathrm{L}}+\overline{D_{\gamma \mathrm{L}}^{-}} \gamma_{\mu} D_{\delta \mathrm{L}}\right)$ & M \\
\hline $\mathcal{O}^{6}$ & $\left(\overline{L_{\alpha}} \tilde{H} \gamma^{\mu} \tilde{H}^{\dagger} L_{\beta}\right)\left(\overline{L_{\gamma}} \gamma_{\mu} L_{\delta}\right)$ & $\left(\nu_{\alpha \mathrm{L}}^{-} \gamma^{\mu} \nu_{\beta \mathrm{L}}\right)\left({\overline{\nu_{\gamma \mathrm{L}}}}_{\gamma_{\mu}} \nu_{\delta \mathrm{L}}+\overline{E_{\gamma \mathrm{L}}} \gamma_{\mu} E_{\delta \mathrm{L}}\right)$ & M \\
\hline $\mathcal{O}^{7}$ & $\left(\overline{L_{\alpha}} \tilde{H} \gamma^{\mu} L_{b \beta}\right)\left(\overline{Q_{b \gamma}} \gamma_{\mu} \tilde{H}^{\dagger} Q_{\delta}\right)$ & $\left(\nu_{\alpha \mathrm{L}}^{-} \gamma^{\mu} \nu_{\beta \mathrm{L}}\right)\left(\overline{U_{\gamma \mathrm{L}}} \gamma_{\mu} U_{\delta \mathrm{L}}\right)+\left(\nu_{\alpha \mathrm{L}}^{-} \gamma^{\mu} E_{\beta \mathrm{L}}\right)\left(\overline{D_{\gamma \mathrm{L}}} \gamma_{\mu} U_{\delta \mathrm{L}}\right)$ & S,M,D \\
\hline $\mathcal{O}^{8}$ & $\varepsilon_{b c}\left(\bar{L}_{\alpha} \tilde{H} \gamma^{\mu} L_{b \beta}\right)\left(\bar{Q}_{\gamma} H \gamma_{\mu} Q_{c \delta}\right)$ & $\left(\nu_{\alpha \mathrm{L}}^{-} \gamma^{\mu} \nu_{\beta \mathrm{L}}\right)\left(\overline{D_{\gamma \mathrm{L}}^{-}} \gamma_{\mu} D_{\delta \mathrm{L}}\right)-\left(\nu_{\alpha \mathrm{L}}^{-} \gamma^{\mu} E_{\beta \mathrm{L}}\right)\left(\overline{D_{\gamma \mathrm{L}}^{-}} \gamma_{\mu} U_{\delta \mathrm{L}}\right)$ & $\mathrm{S}, \mathrm{M}, \mathrm{D}$ \\
\hline $\mathcal{O}^{9}$ & $\varepsilon_{b c}\left(\bar{L}_{\alpha} \tilde{H} \gamma^{\mu} L_{b \beta}\right)\left(\bar{Q}_{\gamma} H \gamma_{\mu} Q_{c \delta}\right)$ & $\left(\nu_{\alpha \mathrm{L}}^{-} \gamma^{\mu} E_{\beta \mathrm{L}}\right)\left(D_{\underline{\gamma \mathrm{R}}}^{-} \gamma_{\mu} U_{\delta \mathrm{R}}\right)$ & $\mathrm{S}, \mathrm{D}$ \\
\hline $\mathcal{O}^{10}$ & $\left(\overline{L_{\alpha}} \tilde{H} \sigma^{\mu \nu} E_{\beta \mathrm{R}}\right)\left(\bar{Q}_{\gamma} H \sigma_{\mu \nu} U_{\delta \mathrm{R}}\right)$ & $\left(\nu_{\alpha \mathrm{L}}^{-} \sigma^{\mu \nu} E_{\beta \mathrm{R}}\right)\left(D_{\gamma \mathrm{L}}^{-} \sigma_{\mu \nu} U_{\delta \mathrm{R}}\right)$ & S,D \\
\hline $\mathcal{O}^{11}$ & $\left(\overline{L_{\alpha}} \tilde{H} E_{\beta \mathrm{R}}\right)\left({\overline{D_{\gamma \mathrm{R}}}}_{\overline{H^{\dagger}}}^{\dagger} Q_{\delta}\right)$ & $\left(\nu_{\alpha \mathrm{L}}^{-} E_{\beta \mathrm{R}}\right)\left(D_{\gamma \mathrm{R}}^{-} U_{\delta \mathrm{L}}\right)$ & S,D \\
\hline $\mathcal{O}^{12}$ & $\left(\overline{L_{\alpha}} \tilde{H} E_{\beta \mathrm{R}}\right)\left(\bar{Q}_{\gamma} H U_{\delta \mathrm{R}}\right)$ & $\left(\nu_{\alpha \mathrm{L}}^{-} E_{\beta \mathrm{R}}\right)\left(\overline{D_{\gamma \mathrm{L}}^{-}} U_{\delta \mathrm{R}}\right)$ & S,D \\
\hline
\end{tabular}

$$
\begin{aligned}
& c_{e e 11}^{2}=-2 c_{\mu \mu 11}^{2}=-2 c_{\tau \tau 11}^{2}, \quad c_{\alpha \beta 11}^{2}=0 \text { for } \alpha \neq \beta, \\
& c_{\mu \mu 11}^{2}=-c_{\tau \tau 11}^{2}, \quad c_{e e 11}^{2}=c_{\alpha \beta 11}^{2}=0 \text { for } \alpha \neq \beta,
\end{aligned}
$$

respectively, where all nonvanishing values are real. The rest $\left[(\bar{L} L)_{\mathbf{1}^{\prime}}\left(\overline{U_{\mathrm{R}}} U_{\mathrm{R}}\right)_{\mathbf{1}^{\prime \prime}},(\bar{L} L)_{\mathbf{1}^{\prime \prime}}\left(\overline{U_{\mathrm{R}}} U_{\mathrm{R}}\right)_{\mathbf{1}^{\prime}}\right.$, $(\bar{L} L)_{\mathbf{3}_{\mathrm{S}}}\left(\overline{U_{\mathrm{R}}} U_{\mathrm{R}}\right)_{\mathbf{3}_{\mathrm{A}}}$, and $\left.(\bar{L} L)_{\mathbf{3}_{\mathrm{A}}}\left(\overline{U_{\mathrm{R}}} U_{\mathrm{R}}\right)_{\mathbf{3}_{\mathrm{A}}}\right]$ do not contribute to $c_{\alpha \beta 11}^{2}$.

The correlations of the coefficients $c_{\alpha \beta 11}^{2}$ directly determine the flavor structure of matter-effect NSIs. In particular, Eq. (27) directly gives rise to $\mathbb{T}_{11}$, and Eq. (29) leads to $\mathbb{T}_{12}$ and $\mathbb{T}_{13}$. The discussion of $\mathcal{O}_{\alpha \beta \gamma \delta}^{2}$ applies to $\mathcal{O}_{\alpha \beta \gamma \delta}^{3-8}$. In other words, the NSI textures $\mathbb{T}_{11}, \mathbb{T}_{12}$, and $\mathbb{T}_{13}$ can be derived from

$$
(\bar{L} L)_{\mathbf{1}}(\bar{F} F)_{\mathbf{1}}, \quad(\bar{L} L)_{\mathbf{3}_{\mathrm{S}}}(\bar{F} F)_{\mathbf{3}_{\mathrm{s}}}, \quad(\bar{L} L)_{\mathbf{3}_{\mathrm{A}}}(\bar{F} F)_{\mathbf{3}_{\mathrm{s}}},
$$

respectively, where $F$ represents any fermions in the SM.

\section{NSI textures predicted by the residual symmetry of $\boldsymbol{A}_{\mathbf{4}}$}

In order to break $A_{4}$ and obtain residual symmetries, we include the flavon VEV in the NSI operators. We consider that the operators $c_{\alpha \beta \gamma \delta}^{p} \mathcal{O}_{\alpha \beta \gamma \delta}^{p}$ are effectively realized via ${ }^{5}$

$$
c_{\alpha^{\prime} \alpha \beta \gamma \delta}^{\varphi, p} \frac{\varphi_{\alpha^{\prime}}}{v_{\varphi}} \mathcal{O}_{\alpha \beta \gamma \delta \delta}^{p} \quad \text { or } \quad c_{\alpha^{\prime} \alpha \beta \gamma \delta}^{\chi, p} \frac{\chi_{\alpha^{\prime}}}{v_{\chi}} \mathcal{O}_{\alpha \beta \gamma \delta}^{p} .
$$

These operators are $A_{4}$-invariant before flavons get VEVs. Taking the VEVs in Eq. (7), we obtain $c_{\alpha \beta \gamma \delta}^{p} \mathcal{O}_{\alpha \beta \gamma \delta}^{p}$ with

\footnotetext{
${ }^{5}$ Since the conjugates of $\varphi$ and $\chi$ are identical to $\varphi$ and $\chi$, respectively, it is not necessary to write out operators realized by $\varphi^{*}$ or $\chi^{*}$ separately.
}

$$
c_{\alpha \beta \gamma \delta}^{p}=c_{1 \alpha \beta \gamma \delta}^{\varphi, p} \quad \text { or } \quad c_{1 \alpha \beta \gamma \delta}^{\chi, p}+c_{2 \alpha \beta \gamma \delta}^{\chi, p}+c_{3 \alpha \beta \gamma \delta}^{\chi, p} .
$$

They are no longer $A_{4}$-invariant, but they only preserve a $Z_{3}$ or $Z_{2}$ symmetry, since $\varphi$ and $\chi$ preserve $Z_{3}$ and $Z_{2}$ symmetries, respectively. The $Z_{3}$-invariant operators $\varphi \mathcal{O}$ do not give any new information, and we recover Eq. (22). The reason is that the generator of $Z_{3}, \mathcal{T}$, is diagonal, and the predicted NSI textures must also be diagonal. In the following, we will not consider the $Z_{3}$-invariant operator $\varphi \mathcal{O}$ anymore.

Now we focus on the $A_{4}$-breaking $Z_{2}$-invariant operators $\chi \mathcal{O}$. We first define the following nondiagonal textures:

$$
\begin{array}{rlrl}
\mathbb{T}_{21} & =\left(\begin{array}{lll}
0 & 1 & 1 \\
1 & 0 & 1 \\
1 & 1 & 0
\end{array}\right), & \mathbb{T}_{22}=\left(\begin{array}{ccc}
0 & -1 & -1 \\
-1 & 0 & 2 \\
-1 & 2 & 0
\end{array}\right), \\
\mathbb{T}_{23}=\left(\begin{array}{ccc}
0 & -1 & 1 \\
-1 & 0 & 0 \\
1 & 0 & 0
\end{array}\right), & \\
\mathbb{T}_{31}=\left(\begin{array}{ccc}
0 & -i & i \\
i & 0 & -i \\
-i & i & 0
\end{array}\right), & \mathbb{T}_{32}=\left(\begin{array}{ccc}
0 & i & -i \\
-i & 0 & -2 i \\
i & 2 i & 0
\end{array}\right), \\
\mathbb{T}_{33}=\left(\begin{array}{ccc}
0 & i & i \\
-i & 0 & 0 \\
-i & 0 & 0
\end{array}\right) . &
\end{array}
$$

$\mathbb{T}_{2 n}$ represent nondiagonal real NSI textures, while $\mathbb{T}_{3 n}$ represent pure imaginary NSI textures.

For $c_{\alpha^{\prime} \alpha \beta \gamma \delta}^{\chi, 1} \chi_{\alpha^{\prime}} \mathcal{O}_{\alpha \beta \gamma \delta}^{1}$, there are nine $Z_{2}$-invariant operators that can contribute to NSIs: 
$\chi(\bar{L} L)_{\mathbf{3}_{\mathrm{S}}}(\bar{L} L)_{\mathbf{1}}, \quad \chi(\bar{L} L)_{\mathbf{3}_{\mathrm{s}}}(\bar{L} L)_{\mathbf{1}^{\prime}}, \quad \chi(\bar{L} L)_{\mathbf{3}_{\mathrm{S}}}(\bar{L} L)_{\mathbf{1}^{\prime \prime}}$,

$\chi(\bar{L} L)_{\mathbf{3}_{\mathrm{A}}}(\bar{L} L)_{\mathbf{1}}, \quad \chi(\bar{L} L)_{\mathbf{3}_{\mathrm{A}}}(\bar{L} L)_{\mathbf{1}^{\prime}}, \quad \chi(\bar{L} L)_{\mathbf{3}_{\mathrm{A}}}(\bar{L} L)_{\mathbf{1}^{\prime \prime}}$,

$\chi\left((\bar{L} L)_{\mathbf{3}_{\mathrm{S}}}(\bar{L} L)_{\mathbf{3}_{\mathrm{S}}}\right)_{\mathbf{3}_{\mathrm{S}}}, \quad \chi\left((\bar{L} L)_{\mathbf{3}_{\mathrm{A}}}(\bar{L} L)_{\mathbf{3}_{\mathrm{A}}}\right)_{\mathbf{3}_{\mathrm{S}}}$,

$\chi\left((\bar{L} L)_{\mathbf{3}_{\mathrm{S}}}(\bar{L} L)_{\mathbf{3}_{\mathrm{A}}}\right)_{\mathbf{3}_{\mathrm{s}}}, \quad \chi\left((\bar{L} L)_{\mathbf{3}_{\mathrm{S}}}(\bar{L} L)_{\mathbf{3}_{\mathrm{A}}}\right)_{\mathbf{3}_{\mathrm{A}}}$.

Because both $\alpha$ and $\gamma$, and $\beta$ and $\delta$ are antisymmetric, $c_{e \beta 11}^{1}=c_{\alpha e 11}^{1}=0$ for all cases. The other coefficients satisfy the following relations, respectively. Taking the CG coefficients in Eq. (4) into account, we obtain

$$
2 c_{\mu \mu 11}^{1}=2 c_{\tau \tau 11}^{1}=c_{\mu \tau 11}^{1}=c_{\tau \mu 11}^{1}
$$

for $\quad \chi\left((\bar{L} L)_{\mathbf{3}_{\mathrm{S}}}(\bar{L} L)_{\mathbf{1}, \mathbf{1}^{\prime}, \mathbf{1}^{\prime \prime}}\right)_{\mathbf{3}}, \quad \chi\left((\bar{L} L)_{\mathbf{3}_{\mathrm{S}}}(\bar{L} L)_{\mathbf{3}_{\mathrm{s}}}\right)_{\mathbf{3}_{\mathrm{S}}}, \quad$ and $\chi\left((\bar{L} L)_{\mathbf{3}_{\mathrm{A}}}(\bar{L} L)_{\mathbf{3}_{\mathrm{A}}}\right)_{\mathbf{3}_{\mathrm{S}}}$, and

$$
c_{\mu \mu 11}^{1}=-c_{\tau \tau 11}^{1}, \quad c_{\mu \tau 11}^{1}=c_{\tau \mu 11}^{1}=0
$$

for $\quad \chi\left((\bar{L} L)_{\mathbf{3}_{\mathrm{A}}}(\bar{L} L)_{\mathbf{1}, \mathbf{1}^{\prime}, \mathbf{1}^{\prime \prime}}\right)_{\mathbf{3}}, \quad \chi\left((\bar{L} L)_{\mathbf{3}_{\mathrm{S}}}(\bar{L} L)_{\mathbf{3}_{\mathrm{A}}}\right)_{\mathbf{3}_{\mathrm{s}}}, \quad$ and $\chi\left((\bar{L} L)_{\mathbf{3}_{\mathrm{S}}}(\bar{L} L)_{\mathbf{3}_{\mathrm{A}}}\right)_{\mathbf{3}_{\mathrm{A}}}$. The first two relations give

$$
\frac{1}{3}\left(2 \mathbb{T}_{11}-\mathbb{T}_{12}+2 \mathbb{\mathbb { T }}_{21}+2 \mathbb{\mathbb { T }}_{23}\right)=\left(\begin{array}{ccc}
0 & 0 & 0 \\
0 & 1 & 2 \\
0 & 2 & 1
\end{array}\right)
$$

and $\mathbb{T}_{13}$, respectively.

For $c_{\alpha^{\prime} \alpha \beta \gamma \delta}^{\chi, 2} \chi_{\alpha^{\prime}} \mathcal{O}_{\alpha \beta \gamma \delta}^{2}$, i.e., the first dimension-eight operator $\left(\overline{L_{\alpha}} \tilde{H} \gamma^{\mu} \tilde{H}^{\dagger} L_{\beta}\right)\left(\overline{U_{\gamma \mathrm{R}}} \gamma_{\mu} U_{\delta \mathrm{R}}\right)$, depending on the representation of $U_{\mathrm{R}}$, there are several $Z_{2}$-invariant operators:

(1) If $U_{1 \mathrm{R}}$ is a trivial singlet $1, \mathbf{1}^{\prime}$, or $\mathbf{1}^{\prime \prime}$ of $A_{4}$, there are two $Z_{2}$-invariant operators:

$$
\chi(\bar{L} L)_{\mathbf{3}_{\mathrm{S}}}\left(\overline{U_{1 \mathrm{R}}} U_{1 \mathrm{R}}\right)_{\mathbf{1}}, \quad \chi(\bar{L} L)_{\mathbf{3}_{\mathrm{A}}}\left(\overline{U_{1 \mathrm{R}}} U_{1 \mathrm{R}}\right)_{\mathbf{1}}
$$

They lead to the following relations of the coefficients:

$$
\begin{aligned}
c_{e e 11}^{2} & =c_{\mu \tau 11}^{2}=c_{\tau \mu 11}^{2}=-2 c_{\mu \mu 11}^{2}=-2 c_{\tau \tau 11}^{2} \\
& =-2 c_{e \mu 11}^{2}=-2 c_{\mu e 11}^{2}=-2 c_{e \tau 11}^{2}=-2 c_{\tau e 11}^{2} ; \\
-c_{\mu \mu 11}^{2} & =c_{\tau \tau 11}^{2}=c_{e \mu 11}^{2}=c_{\mu e 11}^{2}=-c_{e \tau 11}^{2}=-c_{\tau e 11}^{2}, \\
c_{e e 11}^{2} & =c_{e \tau 11}^{2}=c_{\tau e 11}^{2}=0,
\end{aligned}
$$

respectively. They give rise to two textures, $\mathbb{T}_{2} \equiv$ $\mathbb{T}_{12}+\mathbb{T}_{22}$ and $\mathbb{T}_{3} \equiv \mathbb{T}_{13}+\mathbb{T}_{23}$, respectively.

(2) If $U_{1 \mathrm{R}}$ is arranged as one component of a triplet $U_{\mathrm{R}}=\left(U_{1 \mathrm{R}}, U_{2 \mathrm{R}}, U_{3 \mathrm{R}}\right)^{T} \sim \mathbf{3}$ of $A_{4}$, there are six independent $Z_{2}$-invariant operators contributing to NSIs:

$$
\begin{aligned}
& \chi(\bar{L} L)_{\mathbf{3}_{\mathrm{S}}}\left(\overline{U_{\mathrm{R}}} U_{\mathrm{R}}\right)_{\mathbf{1}}, \quad \chi(\bar{L} L)_{\mathbf{3}_{\mathrm{A}}}\left(\overline{U_{\mathrm{R}}} U_{\mathrm{R}}\right)_{\mathbf{1}}, \\
& \chi\left((\bar{L} L)_{\mathbf{3}_{\mathrm{s}}}\left(\overline{U_{\mathrm{R}}} U_{\mathrm{R}}\right)_{\mathbf{3}_{\mathrm{s}}}\right)_{\mathbf{3}_{\mathrm{s}},} \\
& \chi\left((\bar{L} L)_{\mathbf{3}_{\mathrm{s}}}\left(\overline{U_{\mathrm{R}}} U_{\mathrm{R}}\right)_{\mathbf{3}_{\mathrm{S}}}\right)_{\mathbf{3}_{\mathrm{A}}}, \quad \chi\left((\bar{L} L)_{\mathbf{3}_{\mathrm{A}}}\left(\overline{U_{\mathrm{R}}} U_{\mathrm{R}}\right)_{\mathbf{3}_{\mathrm{s}}}\right)_{\mathbf{3}_{\mathrm{s}}}, \\
& \chi\left((\bar{L} L)_{\mathbf{3}_{\mathrm{A}}}\left(\overline{U_{\mathrm{R}}} U_{\mathrm{R}}\right)_{\mathbf{3}_{\mathrm{S}}}\right)_{\mathbf{3}_{\mathrm{A}}} .
\end{aligned}
$$

The first two give the same two correlations as in Eq. (39). The remaining four give rise to

$$
\begin{aligned}
c_{e e 11}^{2} & =-2 c_{\mu \mu 11}^{2}=-2 c_{\tau \tau 11}^{2}=-2 c_{\mu \tau 11}^{2}=-2 c_{\tau \mu 11}^{2} \\
& =4 c_{e \mu 11}^{2}=4 c_{\mu e 11}^{2}=c_{e \tau 11}^{2}=4 c_{\tau e 11}^{2} \\
c_{\mu \mu 11}^{2} & =-c_{\tau \tau 11}^{2}=2 c_{e \mu 11}^{2}=2 c_{\mu e 11}^{2}=-2 c_{e \tau 11}^{2}=2 c_{\tau e 11}^{2} \\
c_{e e 11}^{2} & =c_{e \tau 11}^{2}=c_{\tau e 11}^{2}=0 \\
i c_{\mu \tau 11}^{2} & =-i c_{\tau \mu 11}^{2}=-2 i c_{e \mu 11}^{2}=2 i c_{\mu e 11}^{2} \\
& =2 i c_{e \tau 11}^{2}=-2 i c_{\tau e 11}^{2}, \\
c_{e e 11}^{2} & =c_{\mu \mu 11}^{2}=c_{\tau \tau 11}^{2}=0 ; i c_{e \mu 11}^{2}=-i c_{\mu e 11}^{2} \\
& =i c_{e \tau 11}^{2}=-i c_{\tau e 11}^{2}, \\
c_{e e 11}^{2} & =c_{\mu \mu 11}^{2}=c_{\tau \tau e 11}^{2}=c_{\mu \tau e 11}^{2}=c_{\tau \mu 11}^{2}=0
\end{aligned}
$$

respectively, where all nonvanishing values are real (as required by the Hermitian of the Lagrangian). They give rise to

$$
\begin{aligned}
2 \mathbb{T}_{12}-\mathbb{T}_{22} & =\left(\begin{array}{ccc}
4 & 1 & 1 \\
1 & -2 & -2 \\
1 & -2 & -2
\end{array}\right), \\
2 \mathbb{T}_{13}-\mathbb{T}_{23} & =\left(\begin{array}{ccc}
0 & 1 & -1 \\
1 & 2 & 0 \\
-1 & 0 & -2
\end{array}\right),
\end{aligned}
$$

and $\mathbb{T}_{32}$ and $\mathbb{T}_{33}$, respectively.

A similar discussion applies to $\mathcal{O}^{3-8}$, and the same textures as predicted by $\mathcal{O}^{2}$ are obtained from these operators.

The nine textures $\mathbb{T}_{m n}$ in Eqs. (22) and (33) form a complete basis for a Hermitian $3 \times 3$ matrix. Any two of these textures are orthogonal in the Hilbert-Schmidt inner product, $\operatorname{tr}\left(\mathbb{T}_{m n}^{\dagger} \mathbb{T}_{m^{\prime} n^{\prime}}\right) \propto \delta_{m m^{\prime}} \delta_{n n^{\prime}}$. Matter-effect NSIs contribute to the effective Hamiltonian term via the matrix

$$
\begin{aligned}
\epsilon & \equiv\left(\begin{array}{ccc}
\epsilon_{e e} & \epsilon_{e \mu} & \epsilon_{e \tau} \\
\epsilon_{\mu e} & \epsilon_{\mu \mu} & \epsilon_{\mu \tau} \\
\epsilon_{\tau e} & \epsilon_{\tau \mu} & \epsilon_{\tau \tau}
\end{array}\right) \equiv\left(\begin{array}{ccc}
\epsilon_{e e} & \left|\epsilon_{e \mu}\right| \mathrm{e}^{i \phi_{e \mu}} & \left|\epsilon_{e \tau}\right| \mathrm{e}^{i \phi_{e \tau}} \\
\left|\epsilon_{\mu e}\right| \mathrm{e}^{-i \phi_{e \mu}} & \epsilon_{\mu \mu} & \left|\epsilon_{\mu \tau}\right| \mathrm{e}^{i \phi_{\mu \tau}} \\
\left|\epsilon_{e \tau}\right| \mathrm{e}^{-i \phi_{e \tau}} & \left|\epsilon_{\mu \tau}\right| \mathrm{e}^{-i \phi_{\mu \tau}} & \epsilon_{\tau \tau}
\end{array}\right) \\
& =\sum_{m, n=1,2,3} \alpha_{m n} \mathbb{T}_{m n} / N_{m n},
\end{aligned}
$$

where $N_{m n}$ are the normalization factors $N_{11}=\sqrt{3}, N_{12}=$ $\sqrt{6}, N_{13}=\sqrt{2}, N_{21}=N_{31}=\sqrt{6}, N_{22}=N_{32}=2 \sqrt{3}$, and 
TABLE II. NSI textures in matter predicted by $A_{4}$ and the residual symmetry $Z_{2}$, where $F$ represents any SM fermion. The textures $\mathbb{T}_{1 n}$ are defined in Eq. (22), $\mathbb{T}_{2 n}$ and $\mathbb{T}_{3 n}$ are defined in Eq. (33), and $\chi$ is defined in Eq. (7).

\begin{tabular}{|c|c|c|c|}
\hline & Representations & $A_{4}$-invariant operators & NSI textures \\
\hline $\mathcal{O}^{1}$ & $L \sim \mathbf{3}$ & $\begin{array}{l}(\bar{L} L)_{\mathbf{1}}(\bar{L} L)_{\mathbf{1}},(\bar{L} L)_{\mathbf{1}^{\prime}}(\bar{L} L)_{\mathbf{1}^{\prime \prime}} \\
(\bar{L} L)_{\mathbf{3}_{\mathrm{s}}}(\bar{L} L)_{\mathbf{3}_{\mathrm{s}}},(\bar{L} L)_{\mathbf{3}_{\mathrm{A}}}(\bar{L} L)_{\mathbf{3}_{\mathrm{A}}}\end{array}$ & $2 \mathbb{T}_{11}-\mathbb{T}_{12}$ \\
\hline $\mathcal{O}^{2-8}$ & $\begin{array}{l}L \sim \mathbf{3}, F \sim \mathbf{1}, \mathbf{1}^{\prime}, \mathbf{1}^{\prime \prime}, \mathbf{3} \\
L \sim \mathbf{3}, F \sim \mathbf{3}\end{array}$ & $\begin{array}{l}(\bar{L} L)_{\mathbf{1}}(\bar{F} F)_{\mathbf{1}} \\
(\bar{L} L)_{\mathbf{3}_{\mathrm{s}}}(\bar{F} F)_{\mathbf{3}_{\mathrm{s}}} \\
(\bar{L} L)_{\mathbf{3}_{\mathrm{A}}}(\bar{F} F)_{\mathbf{3}_{\mathrm{S}}}\end{array}$ & $\begin{array}{l}\mathbb{T}_{11} \\
\mathbb{T}_{12} \\
\mathbb{T}_{13}\end{array}$ \\
\hline$\chi \mathcal{O}^{1}$ & $\begin{array}{l}\text { Representations } \\
\chi \sim \mathbf{3}, L \sim \mathbf{3}\end{array}$ & $\begin{array}{c}Z_{2} \text {-invariant operators } \\
\chi\left((\bar{L} L)_{\mathbf{3}_{\mathrm{S}}}(\bar{L} L)_{\mathbf{1}, \mathbf{1}^{\prime}, \mathbf{1}^{\prime \prime}}\right)_{\mathbf{3}}, \chi\left((\bar{L} L)_{\mathbf{3}_{\mathrm{S}}}(\bar{L} L)_{\mathbf{3}_{\mathrm{S}}}\right)_{\mathbf{3}_{\mathrm{s}}} \\
\chi\left((\bar{L} L)_{\mathbf{3}_{\mathrm{A}}},(\bar{L} L)_{\mathbf{3}_{\mathrm{A}}}\right)_{\mathbf{3}_{\mathrm{s}}} \\
\chi\left((\bar{L} L)_{\mathbf{3}_{\mathrm{A}}}(\bar{L} L)_{\mathbf{1}, \mathbf{1}^{\prime}, \mathbf{1}^{\prime \prime}}\right)_{\mathbf{3}}, \chi\left((\bar{L} L)_{\mathbf{3}_{\mathrm{S}}}(\bar{L} L)_{\mathbf{3}_{\mathrm{A}}}\right)_{\mathbf{3}_{\mathrm{S}}}\end{array}$ & $\begin{array}{c}\text { NSI textures } \\
\frac{1}{3}\left(2 \mathbb{T}_{11}-\mathbb{T}_{12}+2 \mathbb{T}_{21}+2 \mathbb{T}_{23}\right) \\
\mathbb{T}_{13}\end{array}$ \\
\hline & $\chi \sim \mathbf{3}, L \sim \mathbf{3}, F \sim \mathbf{1}, \mathbf{1}^{\prime}, \mathbf{1}^{\prime \prime}, \mathbf{3}$ & $\begin{array}{c}\chi(\bar{L} L)_{\mathbf{3}_{\mathrm{s}}}(\bar{F} F)_{\mathbf{1}} \\
\chi(\bar{L} L)_{\mathbf{3}_{\mathrm{A}}}(\bar{F} F)_{\mathbf{1}}\end{array}$ & $\begin{array}{l}\mathbb{T}_{12}+\mathbb{T}_{22} \\
\mathbb{T}_{13}+\mathbb{T}_{23}\end{array}$ \\
\hline$\chi \mathcal{O}^{2-8}$ & $\chi \sim \mathbf{3}, L \sim \mathbf{3}, F \sim \mathbf{3}$ & $\begin{array}{l}\chi\left((\bar{L} L)_{\mathbf{3}_{\mathrm{S}}}(\bar{F} F)_{\mathbf{3}_{\mathrm{s}}}\right)_{\mathbf{3}_{\mathrm{S}}} \\
\chi\left((\bar{L} L)_{\mathbf{3}_{\mathrm{A}}}(\bar{F} F)_{\mathbf{3}_{\mathrm{S}}}\right)_{\mathbf{3}_{\mathrm{s}}} \\
\chi\left((\bar{L} L)_{\mathbf{3}_{\mathrm{S}}}(\bar{F} F)_{\mathbf{3}_{\mathrm{s}}}\right)_{\mathbf{3}_{\mathrm{A}}} \\
\chi\left((\bar{L} L)_{\mathbf{3}_{\mathrm{A}}}(\bar{F} F)_{\mathbf{3}_{\mathrm{s}}}\right)_{\mathbf{3}_{\mathrm{A}}}\end{array}$ & $\begin{array}{c}2 \mathbb{T}_{12}-\mathbb{T}_{22} \\
2 \mathbb{T}_{13}-\mathbb{T}_{23} \\
\mathbb{T}_{32} \\
\mathbb{T}_{33}\end{array}$ \\
\hline
\end{tabular}

$N_{23}=N_{33}=2$. The relations between $\epsilon_{\alpha \beta}$ and $\alpha_{m n}$ are shown in Table III, and the following properties are satisfied:

$$
\operatorname{tr}\left(\epsilon \epsilon^{\dagger}\right)=\sum_{\alpha, \beta=e, \mu, \tau}\left|\epsilon_{\alpha \beta}\right|^{2}=\sum_{m, n=1,2,3} \alpha_{m n}^{2}
$$

Note that $\mathbb{T}_{11} \equiv \mathbb{1}$ is unobservable in neutrino oscillations experiments.

We list all $A_{4^{-}}$and $Z_{2}$-motivated matter-effect NSI textures predicted by $A_{4^{-}}$and $Z_{2}$-invariant operators $\mathcal{O}^{p}$ and $\chi \mathcal{O}^{p}$ in Table II, where $\chi$ is the flavon VEV inducing $A_{4}$ breaking to $Z_{2}$. As seen in the table, an NSI texture predicted by an $A_{4}$-invariant ( $Z_{2}$-invariant) operator usually does not preserve $A_{4}\left(Z_{2}\right)$. This is because the matter-effect NSIs have specified the first-generation charged fermions. These charged fermions, if not arranged as a singlet 1 of $A_{4}$, are not invariant in $A_{4}\left(Z_{2}\right)$, and thus the NSI texture does not respect $A_{4}\left(Z_{2}\right)$. In a specific $A_{4}$ model, the NSI matrix $\epsilon$ could be a linear combination of $\mathbb{T}_{m n}$. However, it is notable that $\mathbb{T}_{31}$ cannot be obtained directly from the above analysis. The analysis based on higher-dimensional operators cannot determine which texture is more important and dominant in oscillation experiments. However, as we will discuss in the next section, once we consider UV completion for these textures and include experimental constraints, some of them are suppressed and cannot be measured in neutrino experiments.

\section{NSI TEXTURES REALIZED IN RENORMALIZABLE FLAVOR MODELS}

In this section, we consider how to realize higherdimensional operators in UV-complete models. We follow the widely used technique in Refs. [16,17], where the dimension-six operator is mediated by singly charged gauge-singlet scalars and the dimension-eight operators can be realized with the help of singly charged gaugesinglet scalars and neutral fermions. Imposing the $A_{4}$ symmetry changes the analysis in the following ways. 1) It requires extending the heavy particles as relevant multiplets of $A_{4}$. 2) The mass matrices of these particles gain special structures constrained by $A_{4}$ or $Z_{2}$ (if the $Z_{2}$-invariant flavon VEV $\chi$ is included), which further contribute to the NSI structure. 3) Although experimental

TABLE III. Expressions for the conventional parameters $\epsilon_{\alpha \beta}$ in terms of the texture parameters $\alpha_{m n}$ according to Eqs. (22), (33), and (43).

\begin{tabular}{lc}
\hline \hline$\tilde{\epsilon}_{e e}\left(\equiv \epsilon_{e e}-\epsilon_{\mu \mu}\right)$ & $3 \alpha_{12} / \sqrt{6}-\alpha_{13} / \sqrt{2}$ \\
$\tilde{\epsilon}_{\tau \tau}\left(\equiv \epsilon_{\tau \tau}-\epsilon_{\mu \mu}\right)$ & $-2 \alpha_{13} / \sqrt{2}$ \\
$\epsilon_{e \mu}$ & $\alpha_{21} / \sqrt{6}-\alpha_{22} / \sqrt{12}-\alpha_{23} / 2+i\left(-\alpha_{31} / \sqrt{6}+\alpha_{32} / \sqrt{12}+\alpha_{33} / 2\right)$ \\
$\epsilon_{e \tau}$ & $\alpha_{21} / \sqrt{6}-\alpha_{22} / \sqrt{12}+\alpha_{23} / 2+i\left(\alpha_{31} / \sqrt{6}-\alpha_{32} / \sqrt{12}+\alpha_{33} / 2\right)$ \\
$\epsilon_{\mu \tau}$ & $\alpha_{21} / \sqrt{6}+2 \alpha_{22} / \sqrt{12}+i\left(-\alpha_{31} / \sqrt{6}-\alpha_{32} / \sqrt{12}\right)$ \\
\hline \hline
\end{tabular}


constraints on the heavy particles have been studied in Refs. [16,17] and later work (e.g., Refs. [18,53]), the nonAbelian flavor symmetry connects channels of different flavors and may result in stronger constraints. Due to these differences, NSIs with $A_{4}$-invariant UV completion deserve a careful consideration.

\section{A. UV completion of the dimension-six operator}

We first consider the UV completion of $\mathcal{O}^{1}$, $\varepsilon_{a c} \varepsilon_{b d}\left(\overline{L_{a \alpha}} \gamma^{\mu} L_{b \beta}\right)\left(\overline{L_{c \gamma}} \gamma_{\mu} L_{d \delta}\right)$. The only way to do this is to introduce a singly charged scalar $S$ which is a $S U(2)_{\mathrm{L}}$ singlet with $Y=+1$ and assume that it couples to $L$ in an "antisymmetric" form [16]. Together with the kinetic and mass terms of $S$, we write down the renormalizable Lagrangian terms as

$$
\begin{aligned}
\mathcal{L}_{S}= & \left(D_{\mu} S\right)^{\dagger}\left(D^{\mu} S\right)-\left(M_{S}^{2}\right)_{\alpha \beta} S_{\alpha}^{*} S_{\beta} \\
& +\lambda_{\alpha \beta \gamma} \varepsilon_{a b} \overline{L_{a \alpha}^{\mathrm{C}}} L_{b \beta} S_{\gamma}+\text { H.c. },
\end{aligned}
$$

where $\lambda_{\alpha \beta \gamma}=-\lambda_{\beta \alpha \gamma}$. In the framework of $A_{4}, S$ cannot be arranged as a singlet representation $\left(\mathbf{1}, \mathbf{1}^{\prime}\right.$, or $\left.\mathbf{1}^{\prime \prime}\right)$ of $A_{4}$ since the symmetric CG coefficients of $A_{4}$ and the antisymmetric property of $\lambda$ lead to $S\left(\overline{L^{\mathrm{C}}} L\right)_{\left.\mathbf{1}^{(\prime, \prime)}\right)} \equiv 0$. Similarly, by arranging $S \sim \mathbf{3}$ we obtain $S\left(\overline{L^{\mathrm{C}}} L\right)_{\mathbf{3}_{\mathrm{s}}}=0$. The only term that can contribute to the operator in Eq. (45) is $S\left(\overline{L^{\mathrm{C}}} L\right)_{\mathbf{3}_{\mathrm{A}}}$ for $S \sim \mathbf{3}$. All nonvanishing coefficients satisfy

$\lambda_{123}=\lambda_{231}=\lambda_{312}=-\lambda_{132}=-\lambda_{213}=-\lambda_{321} \equiv \lambda_{0}$.

After $S$ decouples and by using the Fierz identity, we obtain $\mathcal{O}^{1}$ and the resulting NSI parameters are obtained as

$$
\epsilon_{\alpha \beta}^{e}=\frac{1}{\sqrt{2} G_{F}} \lambda_{\beta e}\left(M_{S}^{2}\right)^{-1} \lambda_{\alpha e}^{\dagger},
$$

where each $\lambda_{\alpha \beta}$ is a $1 \times 3$ matrix given by $\lambda_{\alpha \beta}=$ $\left(\lambda_{\alpha \beta 1}, \lambda_{\alpha \beta 2}, \lambda_{\alpha \beta 3}\right)$.

The structures of $\epsilon_{\alpha \beta}^{e}$ are fully determined by the flavor structure of $M_{S}^{2}$. We constrain the $M_{S}^{2}$ structure as follows.

(1) An $A_{4}$-invariant mass term for the charged scalar can only take the form $\mu_{S}^{2}\left(S^{*} S\right)_{1}=\mu_{S}^{2} \sum_{\alpha} S_{\alpha}^{*} S_{\alpha}$, with $\mu_{S}^{2}>0$, leading to the charged scalar mass matrix

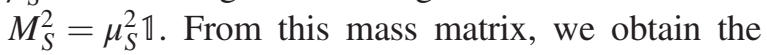
texture $\epsilon^{e}=\alpha_{0} \mathbb{T}_{12}^{\prime}$ with $\alpha_{0}=\frac{\mu_{S}^{2}}{\sqrt{2} G_{F}}$.

(2) In order to obtain nonvanishing off-diagonal NSI entries, $A_{4}$ has to be broken. As shown in the last section, the key is to introduce a flavon with the $Z_{2}$-preserving VEV $\chi$. We add the following renormalizable couplings to the Lagrangian:

$$
\frac{\mu_{S}^{2}}{v_{\chi}}\left[\frac{2}{3} h_{\mathrm{S}}\left(\chi\left(S^{*} S\right)_{\mathbf{3}_{\mathrm{S}}}\right)_{\mathbf{1}}-\frac{2}{\sqrt{3}} h_{\mathrm{A}}\left(\chi\left(S^{*} S\right)_{\mathbf{3}_{\mathrm{A}}}\right)_{\mathbf{1}}\right],
$$

where $h_{\mathrm{S}}$ and $h_{\mathrm{A}}$ are real dimensionless coefficients as required by the Hermiticity of the Lagrangian. Then, the $S$ mass matrix is nondiagonal and the resulting NSI matrix becomes

$$
\begin{aligned}
\epsilon^{e}= & \alpha_{0}\left[\mathbb{T}_{12}^{\prime}+\frac{1}{3}\left(\begin{array}{ccc}
0 & 0 & 0 \\
0 & h_{\mathrm{S}}-h_{\mathrm{S}}^{2} & 2 h_{\mathrm{S}}+h_{\mathrm{S}}^{2} \\
0 & 2 h_{\mathrm{S}}+h_{\mathrm{S}}^{2} & h_{\mathrm{S}}-h_{\mathrm{S}}^{2}
\end{array}\right)\right. \\
& \left.+\frac{1}{3}\left(\begin{array}{ccc}
0 & 0 & 0 \\
0 & \sqrt{3} h_{\mathrm{A}}-h_{\mathrm{A}}^{2} & h_{\mathrm{A}}^{2} \\
0 & h_{\mathrm{A}}^{2} & -\sqrt{3} h_{\mathrm{A}}-h_{\mathrm{A}}^{2}
\end{array}\right)\right],
\end{aligned}
$$

where $\alpha_{0}=\left|\lambda_{0}\right|^{2} /\left[\sqrt{2} G_{F} \mu_{S}^{2}\left(1-h_{\mathrm{S}}^{2}-h_{\mathrm{A}}^{2}\right)\right] . \epsilon^{e}$ contains three real parameters: $\epsilon_{\mu \mu}, \epsilon_{\tau \tau}$, and $\left|\epsilon_{\mu \tau}\right|$. The renormalizable quartic terms $\left((\chi \chi)_{\mathbf{3}_{\mathrm{s}}}\left(S^{*} S\right)_{\mathbf{3}_{\mathrm{s}}}\right)_{\mathbf{1}}$ and $\left((\chi \chi)_{\mathbf{3}_{\mathrm{S}}}\left(S^{*} S\right)_{\mathbf{3}_{\mathrm{A}}}\right)_{\mathbf{1}}$ are also allowed by the symmetry, as such terms do not modify the flavor structures of $M_{S}^{2}$ and $\epsilon^{e}$ except by redefinitions of $h_{\mathrm{S}}$ and $h_{\mathrm{A}}$.

However, it is difficult to realize sizable NSI textures in this approach due to the strong constraint from the radiative charged LFV measurements. Although the tree-level fourcharged-fermion interactions have been avoided, radiative decays $E_{\alpha} \rightarrow E_{\beta} \gamma$ involving $S$ and neutrinos in the loop are triggered by the interaction $\overline{L^{\mathrm{C}}} L S$, and the relative branching ratios are $\propto\left|G_{F}^{-1} \lambda_{\alpha \gamma}\left(M_{S}^{2}\right)^{-1} \lambda_{\beta \gamma}^{\dagger}\right|^{2}$, where $\gamma \neq \alpha, \beta$. The general upper bounds of the $\tau \rightarrow e \gamma$ and $\tau \rightarrow \mu \gamma$ branching ratios are around $10^{-8}$ [54,55], and that of $\mu \rightarrow e \gamma$ is $4.2 \times 10^{-13}$ [56]. Without flavor symmetries, the coefficients $\lambda_{\alpha \beta \gamma}$ and mass terms $\left(M_{S}^{2}\right)_{\alpha \beta}$ are free parameters, and $\tau \rightarrow e \gamma$ and $\mu \rightarrow e \gamma$ do not provide direct constraints on NSIs [16]. Once the flavor symmetry is included, relations such as Eqs. (46) and (48) are satisfied. In the limit $h_{\mathrm{S}}, h_{\mathrm{A}} \rightarrow 0$, all radiative decays are forbidden. However, off-diagonal NSIs are also forbidden in this case, becoming less interesting in oscillation experiments. On the other hand, by assuming $h_{\mathrm{S}}$ or $h_{\mathrm{A}} \sim \mathcal{O}(1)$, the very strong constraint $\left|\epsilon_{\alpha \beta}^{e}\right|<7 \times 10^{-5}$ is obtained from the upper limit of $\mu \rightarrow e \gamma$.

\section{B. UV completions of dimension-eight operators}

In the following, we will only consider NSIs from UV completions of dimension-eight operators. Before performing a detailed analysis, we directly state our main result that in UV-complete models with the $Z_{2}$ residual symmetry only linear combinations of the following NSI textures are worth studying in neutrino oscillation experiments: 


$$
\begin{array}{ll}
\mathbb{T}_{1}=\frac{1}{3}\left(\begin{array}{ccc}
2 & -1 & -1 \\
-1 & 2 & -1 \\
-1 & -1 & 2
\end{array}\right), & \mathbb{T}_{2}=\frac{1}{3}\left(\begin{array}{ccc}
2 & -1 & -1 \\
-1 & -1 & 2 \\
-1 & 2 & -1
\end{array}\right), \\
\mathbb{T}_{3}=\frac{1}{\sqrt{3}}\left(\begin{array}{ccc}
0 & -1 & 1 \\
-1 & 1 & 0 \\
1 & 0 & -1
\end{array}\right), & \mathbb{T}_{4}=\frac{1}{\sqrt{3}}\left(\begin{array}{ccc}
0 & -i & i \\
i & 0 & -i \\
-i & i & 0
\end{array}\right) .
\end{array}
$$

We refer to them as "major NSI textures." They are combinations of some $\mathbb{T}_{m n}, \mathbb{T}_{1}=\frac{1}{3}\left(2 \mathbb{T}_{11}-\mathbb{T}_{21}\right), \mathbb{T}_{2}=$ $\frac{1}{3}\left(\mathbb{T}_{12}+\mathbb{T}_{22}\right), \mathbb{T}_{3}=\frac{1}{\sqrt{3}}\left(\mathbb{T}_{13}+\mathbb{T}_{23}\right)$, and $\mathbb{T}_{4}=\frac{1}{\sqrt{3}} \mathbb{T}_{31}$. As discussed later in this section, the other NSI textures $\mathbb{T}_{12}$, $\mathbb{T}_{13}, \mathbb{T}_{32}, \mathbb{T}_{33}$ and their combinations are strongly constrained by nonoscillation data. Therefore, we call them "minor NSI textures." Here, we classify them into "major" and "minor" due to their testability. In the former case, although they are small, we may still have the opportunity to detect them, while in the later case, we will have no chance to test them in the next-generation neutrino experiments. Throughout this paper, we focus on the "major NSIs textures."

\section{Major NSI textures realized in $U V$-complete $A_{4}$ models}

We consider how to realize the major NSI textures in the renormalizable $A_{4}$ models and consider their experimental constraints. Before electroweak symmetry breaking, the operators $\mathcal{O}^{2-6}$ take the form of a dimension-eight operator $\left(\bar{L} \tilde{H} \gamma^{\mu} \tilde{H}^{\dagger} L\right)\left(\bar{F} \gamma_{\mu} F\right)$. A popular way to realize large NSIs is to introduce a vector boson $Z^{\prime}$. Then, the four-chargedfermion interaction $\left(\bar{F} \gamma^{\mu} F\right)\left(\bar{F} \gamma_{\mu} F\right)$ is unavoidable. In order to be consistent with experimental data, the coupling must be very small. Here, we will carefully avoid the four-charged-fermion interactions introduced after the decoupling of the new particles in the UV sector. Thus, interactions mediated by $Z^{\prime}$ will not be considered.

We focus on $\mathcal{O}^{4}$ by using a singly charged scalar $\phi$ and a neutral fermion $N$ to realize major NSI textures. The renormalizable interactions are given by

$$
\begin{aligned}
\mathcal{L}_{\phi, N}= & \left(D_{\mu} \phi\right)^{\dagger}\left(D^{\mu} \phi\right)-\left(M_{\phi}^{2}\right)_{\alpha \beta} \phi_{\alpha}^{*} \phi_{\beta}+\bar{N} i \not N \\
& -M_{N \alpha \beta} \overline{N_{\alpha \mathrm{R}}} N_{\beta \mathrm{L}}-\kappa_{\alpha \beta \gamma} \overline{E_{\alpha \mathrm{R}}} N_{\beta \mathrm{L}} \phi_{\gamma}^{*} \\
& -y_{\alpha \beta} \overline{L_{\alpha}} \tilde{H} N_{\beta \mathrm{R}}+\text { H.c. },
\end{aligned}
$$

where $D_{\mu}=\partial_{\mu}+i e A_{\mu}$. The charged scalar is a $S U(2)_{\mathrm{L}}$ singlet with $Y=-1$. In order to distinguish it from $S$ in the last subsection, we denote it as $\phi$. There is no LNV coupling in the above interactions. For the neutral fermion $N$, we require a vector-like mass term $M_{N} \overline{N_{\mathrm{R}}} N_{\mathrm{L}}$ as shown above. If there is an additional small LNV mass term $\mu \overline{N_{\mathrm{L}}^{\mathrm{C}}} N_{\mathrm{L}}$ and hierarchical masses $y / \sqrt{G_{F}} \ll M_{N}$, we recover the inverse seesaw model [57]. But here we do not specify whether $N$ is related to the origin of active neutrino masses. Regardless of whether there is a small LNV mass term, we can always arrive at a dimension-eight operator $\sim \frac{\kappa^{2} y^{2}}{M_{\phi}^{2} M_{N}^{2}}\left(\bar{L} \tilde{H} E_{\mathrm{R}}\right)\left(\overline{E_{\mathrm{R}}} \tilde{H}^{\dagger} L\right)$ after the decoupling of the charged scalar and sterile neutrinos, from which we obtain $\mathcal{O}^{4}$. Once the flavor structure is included, the $3 \times 3$ NSI parameter matrix $\epsilon^{e}$ is expressed as

$$
\epsilon^{e}=\frac{1}{8 G_{F}^{2}}\left(y M_{N}^{-1} \kappa_{e}\right)\left(M_{\phi}^{2}\right)^{-1}\left(y M_{N}^{-1} \kappa_{e}\right)^{\dagger},
$$

where $\kappa_{e}$ is a $3 \times 3$ matrix defined via $\left(\kappa_{\alpha}\right)_{\beta \gamma}=\kappa_{\alpha \beta \gamma}$ for $\alpha=e, \mu, \tau$.

We now discuss how the $A_{4}$ symmetry can constrain NSIs originating from this renormalizable model. We first consider $A_{4}$-motivated NSI textures without the involvement of flavons. In the flavor space, since we have arranged $L \sim 3$, the fields $N_{\mathrm{L}}, N_{\mathrm{R}}$, and $\phi$ must be triplets to ensure the invariance of the Lagrangian in $A_{4}$. We follow the setup of most $A_{4}$ models in which $E_{1 \mathrm{R}}$ is fixed as a singlet 1 of $A_{4}$. An $A_{4}$-invariant mass term for the charged scalar can only take the form $\mu_{\phi}^{2}\left(\phi^{*} \phi\right)_{1}=\mu_{\phi}^{2} \sum_{i} \phi_{i}^{*} \phi_{i}$, with $\mu_{\phi}^{2}>0$, i.e., the

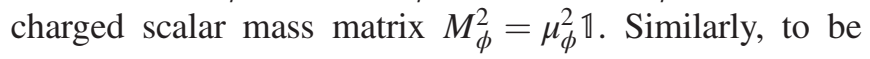
invariant under transformations of $A_{4}$, the Dirac mass matrix of the sterile neutrinos $M_{N}$ and the Yukawa coupling between $L$ and $N_{\mathrm{R}}, y$ is also proportional to the identity matrix, $M_{N}=\mu_{N} \mathbb{1}, y=y_{0} \mathbb{1}$. The structures of the couplings $y$ and $\kappa$ depend on the representations of $E_{\mathrm{R}}$. Interactions involving $\phi$ and $N$ are given by

$$
\kappa_{0} \overline{E_{1 \mathrm{R}}}\left(N_{\mathrm{L}} \phi^{*}\right)_{\mathbf{1}}+y_{0}\left(\bar{L} \tilde{H} N_{\mathrm{R}}\right)_{\mathbf{1}}+\text { H.c. }
$$

Thus, both coupling matrices $\kappa$ and $y$ appear to be proportional to the identity matrix: $\kappa=\kappa_{0} \mathbb{1}$ and $y=y_{0} \mathbb{1}$. After $\phi$ and $N$ are integrated out of the Lagrangian, we find that the $\mathcal{O}^{4}$ takes the form $(\bar{L} L)_{\mathbf{1}}(\bar{F} F)_{1}$, as listed in Table II for $F=E_{\mathrm{R}}$. Finally, we obtain the NSI texture $\epsilon^{e}=\alpha_{0} \mathbb{1}$, where

$$
\alpha_{0}=\frac{\left|y_{0} \kappa_{0}\right|^{2}}{8 G_{F}^{2} \mu_{N}^{2} \mu_{\phi}^{2}} .
$$

Since 1 is the identity matrix, $\epsilon^{e}$ in this special case has no observable signatures in neutrino oscillation experiments.

The involvement of $\chi$ breaks $A_{4}$ to $Z_{2}$ and modifies the correlation relations of the NSI parameters. In order to realize relatively large and measurable NSI effects, we only consider the contribution of renormalizable couplings of $\chi$. There are cases [as shown in Figs. 2(b) and 2(c)] where $\chi$ couples to $\phi$ and $N$ and modifies their mass matrices. 


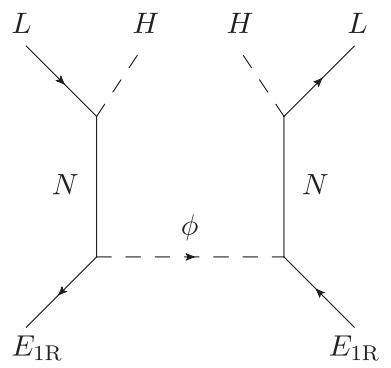

(a)

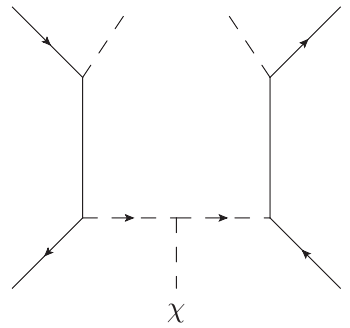

(b)

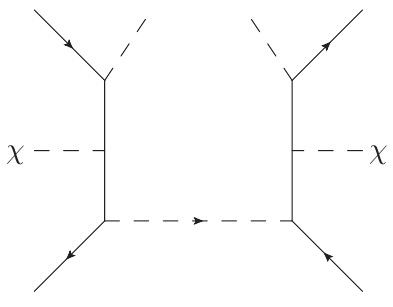

(c)

FIG. 2. Diagrams that give rise to sizable NSI textures corresponding to the dimension-eight operator $\mathcal{O}^{4}$ in leptonic $A_{4}$ models.

(1) The charged scalar $\phi$ mass matrix is modified by the coupling between $\chi$ and $\phi$. We add the following renormalizable coupling to the Lagrangian:

$\frac{\mu_{\phi}^{2}}{v_{\chi}}\left[\frac{2}{3} f_{\mathrm{S}}\left(\chi\left(\phi^{*} \phi\right)_{\mathbf{3}_{\mathrm{S}}}\right)_{\mathbf{1}}-\frac{2}{\sqrt{3}} f_{\mathrm{A}}\left(\chi\left(\phi^{*} \phi\right)_{\mathbf{3}_{\mathrm{A}}}\right)_{\mathbf{1}}\right]$,

where $f_{\mathrm{S}}$ and $f_{\mathrm{A}}$ are real dimensionless coefficients as required by the Hermiticity of the Lagrangian. The relevant higher-dimensional operators after $\phi$ and $N$ are integrated out take the forms $\chi(\bar{L} L)_{\mathbf{3}_{\mathrm{S}}}(\bar{F} F)_{\mathbf{1}}$ and $\chi(\bar{L} L)_{\mathbf{3}_{\mathrm{A}}}(\bar{F} F)_{\mathbf{1}}$, respectively. The modified $\phi$ mass matrix turns out to be

$$
M_{\phi}^{2} / \mu_{\phi}^{2}=\mathbb{1}+f_{\mathrm{S}} \mathbb{T}_{2}+f_{\mathrm{A}} \mathbb{\mathbb { T }}_{3} .
$$

Terms such as $\left((\chi \chi)_{\mathbf{3}_{\mathrm{S}}}\left(\phi^{*} \phi\right)_{\mathbf{3}_{\mathrm{S}}}\right)_{\mathbf{1}},\left((\chi \chi)_{\mathbf{3}_{\mathrm{S}}}\left(\phi^{*} \phi\right)_{\mathbf{3}_{\mathrm{A}}}\right)_{\mathbf{1}}$ are also renormalizable and should be considered for completeness. These terms will not induce new structures different from Eq. (56).

(2) The Dirac mass matrix of $N$ is modified by couplings between $\chi$ and $N$. The related renormalizable Lagrangian term is given by

$$
\begin{aligned}
\frac{\mu_{N}}{v_{\chi}} & {\left[\frac{2}{3} g_{\mathrm{S}}\left(\chi\left(\overline{N_{\mathrm{L}}} N_{\mathrm{R}}\right)_{\mathbf{3}_{\mathrm{S}}}\right)_{\mathbf{1}}-\frac{2}{\sqrt{3}} g_{\mathrm{A}}\left(\chi\left(\overline{N_{\mathrm{L}}} N_{\mathrm{R}}\right)_{\mathbf{3}_{\mathrm{A}}}\right)_{\mathbf{1}}\right] } \\
\quad+\text { H.c. } &
\end{aligned}
$$

where $g_{\mathrm{S}}$ and $g_{\mathrm{A}}$ are in general complex parameters. The Dirac mass matrix $M_{N}$ is modified as

$$
M_{N} / \mu_{N}=\mathbb{1}+g_{\mathrm{S}} \mathbb{\mathbb { T }}_{2}+g_{\mathrm{A}} \mathbb{\mathbb { T }}_{3} .
$$

Taking the flavon-modified mass matrices of $\phi$ and $N$ into account, we state that the final detectable (i.e., ignoring the undetectable 1 ) NSI matrix $\epsilon^{e}$ in Eq. (52) is always a linear combination of $\mathbb{T}_{i}$ for $i=1,2,3,4$. This is guaranteed by the algebra of $\mathbb{T}_{i}$ and can be straightforwardly proven by implying Eqs. (C2) and (C3) in Appendix C. From Table II, one can expect to find the textures $\mathbb{T}_{2}$ and $\mathbb{T}_{3}$. The other two textures, $\mathbb{T}_{1}$ and $\mathbb{T}_{4}$, which do not arise from higherdimensional operators, are obtained from the inverse transformations of $M_{\phi}^{2}$ and $M_{N}$ and the matrix product $\mathbb{T}_{2} \mathbb{T}_{3}=-i \mathbb{\mathbb { T }}_{4} \cdot \mathbb{T}_{1}$ and $\mathbb{T}_{4}$ appear at the second order of $f_{\mathrm{S}}, f_{\mathrm{A}}$ and $g_{\mathrm{S}}, g_{\mathrm{A}}$. If $f_{\mathrm{S}}, f_{\mathrm{A}}, g_{\mathrm{S}}, g_{\mathrm{A}} \ll 1$ is satisfied, the $\mathbb{T}_{1}$ and $\mathbb{T}_{4}$ parts are negligible compared with the $\mathbb{T}_{2}$ and $\mathbb{T}_{3}$ parts. However, these coefficients, as coefficients of renormalizable terms, may take $\mathcal{O}(1)$ values, and thus in this case $\mathbb{T}_{1}$ and $\mathbb{T}_{4}$ may have NSI effects comparable to those of $\mathbb{T}_{2}$ and $\mathbb{T}_{3}$.

The flavor structures of NSIs can be further discussed in the following scenarios, dependent on the role of the flavon VEV $\chi$ :

(1) With the assumption of additional symmetries, $\chi$ may only couple to $\phi$, and not to $N$, i.e., $g_{\mathrm{A}}, g_{\mathrm{S}}=0$. The resulting detectable NSI matrix is explicitly expressed as

$$
\epsilon^{e}=\alpha_{0}\left[\left(f_{\mathrm{S}}^{2}+f_{\mathrm{A}}^{2}\right) \mathbb{T}_{1}-f_{\mathrm{S}} \mathbb{\mathbb { T }}_{2}-f_{\mathrm{A}} \mathbb{\mathbb { T }}_{3}\right] .
$$

Here, only $\mathbb{T}_{1}, \mathbb{T}_{2}$, and $\mathbb{T}_{3}$ appear, and $\alpha_{0}$ has been redefined.

(2) On the other hand, if $\chi$ only couple to $N$, we obtain the following NSI matrix:

$$
\begin{aligned}
\epsilon^{e}= & \alpha_{0}\left\{\left[-\left(2+\left|g_{\mathrm{S}}\right|^{2}+\left|g_{\mathrm{A}}\right|^{2}\right)\left(\left|g_{\mathrm{S}}\right|^{2}+\left|g_{\mathrm{A}}\right|^{2}\right)\right.\right. \\
& \left.+4 \operatorname{Re}\left(g_{\mathrm{S}}^{2}+g_{\mathrm{A}}^{2}\right)+4\left[\operatorname{Im}\left(g_{\mathrm{S}}^{*} g_{\mathrm{A}}\right)\right]^{2}\right] \mathbb{T}_{1} \\
& \left.-2 \operatorname{Re}\left(g_{\mathrm{S}}\right) \mathbb{T}_{2}-2 \operatorname{Re}\left(g_{\mathrm{A}}\right) \mathbb{T}_{3}-2 \operatorname{Im}\left(g_{\mathrm{S}}^{*} g_{\mathrm{A}}\right) \mathbb{T}_{4}\right\},
\end{aligned}
$$

where $\alpha_{0}$ has been redefined. It is a linear combination of all four $\mathbb{T}_{i}$, but $\mathbb{T}_{4}$ is important only if both $\left|g_{\mathrm{S}}\right|$ and $\left|g_{\mathrm{A}}\right|$ are sizable and there is a relative phase between $g_{\mathrm{S}}$ and $g_{\mathrm{A}}$.

(3) If the antisymmetric couplings $f_{\mathrm{A}}$ and $g_{\mathrm{A}}$ are forbidden, the NSI matrix can be simplified to a linear combination of $\mathbb{T}_{1}$ and $\mathbb{T}_{2}$. On the other hand, if the symmetric couplings $f_{\mathrm{S}}$ and $g_{\mathrm{S}}$ are forbidden, the NSI matrix is a linear combination of $\mathbb{T}_{1}$ and $\mathbb{T}_{3}$. These two cases are valid if the group $A_{4}$ is replaced by larger groups. For example, in the hexahedron group $S_{4}$ [58], there are two triplet irreducible representations, and the symmetric and antisymmetric products 
$\mathbf{3}_{\mathrm{S}}$ and $\mathbf{3}_{\mathrm{A}}$ correspond to two different representations. By arranging $\chi$ to be one of the triplets, the antisymmetric (or symmetric) products can be forbidden, and thus only the symmetric (or antisymmetric) couplings are left.

Naively, one may expect that NSIs from the UV completion of the dimension-eight operator are more constrained than those of the dimension-six operator, but this is not the case in the framework of flavor symmetry. First of all, no tree-level CLFV interactions have been introduced by the Lagrangian in Eq. (51), as required. Although radiative CLFV processes are induced by the coupling $\overline{E_{\mathrm{R}}} N_{\mathrm{L}} \phi$, they essentially rely on the coupling with the second- or third-generation charged lepton $E_{2 \mathrm{R}}$ or $E_{3 \mathrm{R}}$. By arranging $E_{1 \mathrm{R}}, E_{2 \mathrm{R}}$, and $E_{3 \mathrm{R}}$ as different singlets of $A_{4}$, the relevant coefficients are theoretically independent of those involved in matter NSIs $[59,60]$. Constraints on CLFV do not apply to NSIs. Regarding collider searches, with a careful treatment of $\phi$ decaying to $e / \mu$ plus missing transverse momentum or $\tau$ plus missing transverse momentum, the existing LEP and LHC data still allow a singlet charged scalar as light as $65 \mathrm{GeV}$ [61]. The main constraint in this model is the bound of the nonunitarity of the lepton mixing. The decoupling of sterile neutrinos contributes to the active neutrino kinetic mixing as $\frac{y^{2}}{M_{N}^{2}}(\bar{L} \tilde{H}) \not \partial\left(\tilde{H}^{\dagger} L\right)$. After rescaling the kinetic terms of active neutrinos, the nonunitarity of the Pontecorvo-Maki-Nakagawa-Sakata (PMNS) matrix is

$\eta \equiv V_{\mathrm{PMNS}}^{\dagger} V_{\mathrm{PMNS}}-\mathbf{1}=\frac{1}{2 \sqrt{2} G_{F}}\left(y M_{N}^{-1}\right)\left(y M_{N}^{-1}\right)^{\dagger}$.

The nonunitarity bound from a global analysis of LFV decays, probes of the universality of weak interactions, Cabibbo-Kobayashi-Maskawa unitarity bounds, and electroweak precision data is around $\eta \sim 10^{-3}$ [52]. Combined with the above constraints, we see that it is still possible to achieve the major NSI textures with coefficients $\sim \eta /\left(G_{F} M_{\phi}^{2}\right)$ at the $10^{-2}$ or $10^{-3}$ level. These values may be measured by the next-generation accelerator neutrino oscillation experiments.

In the above, we have constructed UV-complete models for $\mathcal{O}^{4}$ and $\chi \mathcal{O}^{4}$. A similar discussion can be directly extended to $\mathcal{O}^{2,3,5}$ and $\chi \mathcal{O}^{2,3,5}$ by replacing the singly charged scalar $\phi$ by $\phi_{U_{\mathrm{R}}, D_{\mathrm{R}}, Q}$, which are an $S U(2)_{\mathrm{L}}$ gauge singlet, singlet, and doublet with hypercharges $Y=-2 / 3,+1 / 3$, and $-1 / 6$, respectively, and replacing the singlet $F=E_{1 \mathrm{R}}$ with $F=U_{1 \mathrm{R}}, D_{1 \mathrm{R}}$, and $Q_{1}$, respectively. The resulting NSI matrix is also a linear combination of the textures $\mathbb{T}_{1}, \mathbb{T}_{2}, \mathbb{T}_{3}$, and $\mathbb{T}_{4}$. The textures $\mathbb{T}_{1}, \mathbb{T}_{2}, \mathbb{T}_{3}$, and $\mathbb{T}_{4}$ are obtained by assuming that the charged fermions are singlets of $A_{4}$. This treatment can avoid strong constraints from the second- and third-generation charged fermions. These textures are less constrained than the other textures discussed below, and thus we call them major NSI textures.

\section{Minor NSI textures realized in $U V$-complete $A_{4}$ models}

The minor NSI textures $\mathbb{T}_{12}, \mathbb{T}_{13}, \mathbb{T}_{32}$, and $\mathbb{T}_{33}$ and their combinations cannot be realized in the above discussions. This is compatible with Table II, where the minor textures are obtained by setting $F \sim \mathbf{3}$. To achieve these textures, as shown in Table II, $F$ has to be assumed to be a triplet of $A_{4}$. Then $F$ cannot be chosen as right-handed charged leptons and not realized in the $\mathcal{O}^{4}$ and $\chi \mathcal{O}^{4}$ series. We will discuss how to realize them in UV-complete $A_{4}$ models.

To realize the $A_{4}$-motivated $\mathbb{T}_{12}$ and $\mathbb{T}_{13}$, we choose $F=$ $U_{\mathrm{R}} \equiv\left(U_{1 \mathrm{R}}, U_{2 \mathrm{R}}, U_{3 \mathrm{R}}\right)^{T} \sim \mathbf{3}$ of $A_{4}$ and consider the UV completion of $\mathcal{O}^{2}$. The latter is obtained by replacing the singly charged scalar $\phi$ with a fractionally charged scalar $\phi_{U_{\mathrm{R}}}$, i.e., a scalar leptoquark, with hypercharge $Y=-2 / 3$, and couplings to $N_{\mathrm{L}}$ and $U_{\mathrm{R}}$. The renormalizable couplings are given by

$\kappa_{\mathrm{S}}^{U_{\mathrm{R}}}\left(\left(\overline{U_{\mathrm{R}}} N_{\mathrm{L}}\right)_{\mathbf{3}_{\mathrm{S}}} \phi_{U_{\mathrm{R}}}^{*}\right)_{1}+\kappa_{\mathrm{A}}^{U_{\mathrm{R}}}\left(\left(\overline{U_{\mathrm{R}}} N_{\mathrm{L}}\right)_{\mathbf{3}_{\mathrm{A}}} \phi_{U_{\mathrm{R}}}^{*}\right)_{1}+$ H.c.

Then, the coupling matrix $\kappa$ is modified as $\kappa_{U_{\mathrm{R}}}=\kappa_{\mathrm{S}}^{U_{\mathrm{R}}} \mathbb{T}_{12}+$ $\kappa_{\mathrm{A}}^{U_{\mathrm{R}}} \mathbb{T}_{13}$ and the $A_{4}$-preserved NSI texture

$$
\epsilon^{u} \equiv \frac{1}{8 G_{F}^{2}}\left(y M_{N}^{-1} \kappa_{U_{\mathrm{R}}}\right)\left(M_{\phi_{U_{\mathrm{R}}}}^{2}\right)^{-1}\left(y M_{N}^{-1} \kappa_{U_{\mathrm{R}}}\right)^{\dagger}
$$

is obtained as a linear combination of $\mathbb{T}_{12}$ and $\mathbb{T}_{13}$. Finally, we include the $A_{4}$-breaking effect in the $\phi_{U_{\mathrm{R}}}$ and $N$ mass matrices, as in Eqs. (56) and (58). Nonzero $\mathbb{T}_{32}$ and $\mathbb{T}_{33}$ can be extracted in principle.

The minor textures $\mathbb{T}_{12}, \mathbb{T}_{13}, \mathbb{T}_{32}$, and $\mathbb{T}_{33}$ are expected to receive stronger constraints. The main reason is that $U_{\mathrm{R}}=$ $\left(U_{1 \mathrm{R}}, U_{2 \mathrm{R}}, U_{3 \mathrm{R}}\right)$ is arranged as a triplet of $A_{4}$ and constraints from the second- and third-generation charged fermions should be included. The neutrino kinetic mixing leads to the coupling $\overline{U_{\mathrm{R}}} \nu_{\mathrm{L}} \phi_{U_{\mathrm{R}}}^{*}$. It further modifies the SM predictions of certain processes, e.g., (semi)leptonic decays $U_{\alpha} \rightarrow U_{\beta} \nu \bar{\nu}$ at tree level, radiative decays $U_{\alpha} \rightarrow U_{\beta} \gamma \gamma$ at loop level, and flavor-changing neutral-current processes $U_{\alpha} \rightarrow U_{\beta} \overline{U_{\gamma}} U_{\delta}$ at loop level. As a consequence, precision measurements of charm mesons and baryons can give strong constraints on $\epsilon^{u}$. A detailed discussion of these constraints is the subject of this paper. Realizations of sizable NSI textures $\mathbb{T}_{12}, \mathbb{T}_{13}, \mathbb{T}_{32}$, and $\mathbb{T}_{33}$ via UV completions of the other dimension-eight operators are also hard. Those via $\mathcal{O}^{3,5,7,8}$ gain strong constraints from $K$ and $B$ decays, and those via $\mathcal{O}^{6}$ gain constraints from $E_{\alpha} \rightarrow$ $E_{\beta} \gamma$ decays. Since it is hard to generate sizable NSI for 
textures $\mathbb{T}_{12}, \mathbb{T}_{13}, \mathbb{T}_{32}$, and $\mathbb{T}_{33}$ or their combinations, we refer to them as minor NSI textures.

\section{TESTING NSI TEXTURES AT LBL EXPERIMENTS}

Long-baseline experiments with wide-band beams and sizable matter effects are expected to measure more than one $\epsilon_{\alpha \beta}$, which implies that the flavor dependence of NSIs $\epsilon_{\alpha \beta}$ can be tested. As a result, an experiment of this kind is able to study the flavor symmetry model through the operators $\mathcal{O}^{1-8}$. In this section we will study the matter NSI effects for the DUNE experiment under the flavor symmetry $A_{4}$ or $Z_{2}$. We summarize the connection between the texture parameters $\alpha_{m n}$ and the conventional parameters $\epsilon_{\alpha \beta}$ in Table III. There are some benefits to considering matter-effect NSIs under flavor symmetries. When we assume that $A_{4}$ symmetry is not broken, only two types of NSIs can be seen, both of which are flavor conserving. If $A_{4}$ symmetry is broken and the residual $Z_{2}$ symmetry is preserved, there are no such benefits as all textures are predicted under this symmetry, until we impose a UVcomplete model. Therefore, we expect good performance from DUNE in studying these scenarios. We test the NSI textures from the $A_{4}$ symmetry without assuming any UVcomplete model in Sec. V B. In Sec. V C, we study the $Z_{2}$ testing, following the discussion in Sec. IV B. The approximation to oscillation probabilities with NSI matter effects is presented in Appendix D; the true values used for the oscillation parameters throughout the simulation in this section are given in Table IX.

The current global fit for matter-effect NSIs [23] includes solar, atmospheric, reactor, and LBL neutrino data. With the assumption that all NSIs come entirely from up quarks or down quarks to avoid NSIs at the source and the detector, the current global fit to the standard NSI parameters $\epsilon_{\alpha \beta}^{u}$ and $\epsilon_{\alpha \beta}^{d}$ was performed in Ref. [23]. We adopt these results to estimate the bounds for $\alpha_{m n}^{u, d}$. We only take the bound for each $\epsilon_{\alpha \beta}^{u, d}$, i.e., the results of a $1 \mathrm{D}$ projection. Furthermore, we neglect underlying corrections between any two or among more than two parameters, which are $\epsilon_{\alpha \beta}$, mixing angels, or mass-squared differences. Assuming Gaussian distributions and taking the $90 \%$ C.L. limits from Ref. [23], the bounds on $\epsilon_{\alpha \beta}^{u, d}$ at $1 \sigma$ are shown in Table IV. Since in their analysis the imaginary part was assumed to be 0 or $\pi$, we directly translate their bounds to $\alpha_{1 n}^{u, d}$ and $\alpha_{2 n}^{u, d}$ by setting the imaginary $\alpha_{3 n}^{u, d}=0$, and the results are shown in Table V. NSIs with down quarks $\epsilon_{\alpha \beta}^{u, d}$ have very similar constraints as those with $\epsilon_{\alpha \beta}^{u, d}$. As we neglect some correlations among the parameters, our results can be viewed as optimal. In Table V, we see that most parameters are constrained around or below the percent level of weak interactions, except for $\alpha_{12}^{u, d}$, for which $1 \sigma$ bounds are around $15 \%$.
TABLE IV. Taken from the current global fit results [23] for $\epsilon_{\alpha \beta}^{u}$ and $\epsilon_{\alpha \beta}^{d}$. In these results, the authors [27] assume that off-diagonal elements $\epsilon_{\alpha \neq \beta}$ are real, consider that NSIs is only contributed by $u$ (d) quarks for $\epsilon_{\alpha \beta}^{u}\left(\epsilon_{\alpha \beta}^{d}\right)$, but do not include NSIs at the source and the detector.

\begin{tabular}{llll}
\hline \hline \multicolumn{4}{l}{$\sigma$ bounds of global fit results } \\
\\
\hline$\tilde{\epsilon}_{e e}^{u}$ & {$[0.188,0.376]$} & $\tilde{\epsilon}_{e e}^{d}$ & {$[0.203,0.384]$} \\
$\tilde{\epsilon}_{\tau \tau}^{u}$ & {$[-0.003,0.012]$} & $\tilde{\epsilon}_{\tau \tau}^{d}$ & {$[-0.003,0.012]$} \\
$\epsilon_{e \mu}^{u}$ & {$[-0.046,0.002]$} & $\epsilon_{e \mu}^{d}$ & {$[-0.048,0]$} \\
$\epsilon_{e \tau}^{u}$ & {$[-0.038,0.065]$} & $\epsilon_{e \tau}^{d}$ & {$[-0.036,0.066]$} \\
$\epsilon_{\mu \tau}^{u}$ & {$[-0.004,0.003]$} & $\epsilon_{\mu \tau}^{d}$ & {$[-0.004,0.003]$} \\
\hline \hline
\end{tabular}

TABLE V. The $1 \sigma$ bounds for $\alpha_{12}^{u}\left(\alpha_{12}^{d}\right), \alpha_{13}^{u}\left(\alpha_{13}^{d}\right)$, and $\alpha_{2 i}^{u}\left(\alpha_{2 i}^{d}\right)$, with fixed $\alpha_{3 i}^{u}=0\left(\alpha_{3 i}^{d}=0\right)$, from the global fit results [23] shown in Table IV. See text for details.

\begin{tabular}{llll}
\hline \hline \multicolumn{3}{l}{$1 \sigma$ bounds by global fit results } & \\
\hline$\alpha_{12}^{u}$ & {$[0.089,0.247]$} & $\alpha_{12}^{d}$ & {$[0.099,0.26]$} \\
$\alpha_{13}^{u}$ & {$[-0.003,0.007]$} & $\alpha_{13}^{d}$ & {$[-0.003,0.007]$} \\
$\alpha_{21}^{u}$ & {$[-0.045,0.049]$} & $\alpha_{21}^{d}$ & {$[-0.045,0.047]$} \\
$\alpha_{22}^{u}$ & {$[-0.037,0.03]$} & $\alpha_{22}^{d}$ & {$[-0.035,0.0302]$} \\
$\alpha_{23}^{u}$ & {$[-0.019,0.096]$} & $\alpha_{23}^{d}$ & {$[-0.0154,0.096]$} \\
\hline \hline
\end{tabular}

The matter-effect NSIs are predicted to be small, as we see in Table IV. Fortunately, DUNE can improve the sensitivity and it is possible to detect these effects. In this section, our goal is to see whether these minor features ${ }^{6}$ appearing in DUNE can provide any extra information about the flavor symmetry. We first discuss how mattereffect NSIs $\alpha_{m n}$ affect neutrino oscillations in DUNE, and then we study the physics capacity for DUNE to test $A_{4}$ symmetry and $Z_{2}$ residual symmetry via NSI measurements. We emphasize that the results in Secs. V B and V C are from a general point of view; we consider all possible correlations by using the conventional parametrization (three mixing angles, one Dirac $C P$ phase, and two mass-squared differences) instead of implementing any possible flavor model for the oscillation parameters. The final note is that for a given model that consistently predicts values for both oscillation and NSI parameters, we should further adopt Wilks' theorem that the $\Delta \chi^{2}$ value for nested hypothesis testing asymptotically follows a $\chi^{2}$ distribution, where the number of degrees of freedom is equal to the difference between the number of free parameters in the two models [63]. Therefore, we will further study two cases

\footnotetext{
${ }^{6}$ Assuming an equal amount of NSI effects with $u, d$ quarks and electrons, the $1 \sigma$ size of the total NSI matter effect in the Earth is roughly 3 times that of the $1 \sigma$ region shown Table IV. This estimate will be applied in the following (Tables VI and VIII) for comparison.
} 
with the maximum and minimum possible number of degrees of freedom for a $\chi^{2}$ distribution.

\section{A. Oscillation probabilities in DUNE}

As mentioned in the Introduction, matter-effect NSIs in DUNE have been widely discussed. Because of the long propagation distance $(1300 \mathrm{~km})$ of neutrinos in the Earth, the non-negligible matter density, and the GeV-energyscale neutrino beam, matter effects play a substantial role in oscillations. Before discussing the physics potential of understanding any flavor symmetries, we first study the impact of $\alpha_{m n}$ on the oscillation probability in DUNE.

The DUNE experiment consists of a neutrino source known as the Long Baseline Neutrino Facility (LBNF), a detector based at Fermilab, and a liquid argon timeprojection chamber (LArTPC) detector complex located at Sanford Underground Research Facility a distance of $1300 \mathrm{~km}$ away. The beam design is based on both long baseline neutrino experiment (reference design) and LBNF studies (optimized design). The beam is optimized according to the physics capability of $\delta$ discovery. The $1 \mathrm{MW}$ beam generates a large amount of $\nu_{\mu}\left(\mathrm{POT} / \mathrm{year} \sim 10^{21}\right)$. At the other end, the detector configuration consists of four 10-kiloton LArTPC detectors. LArTPC technology has a
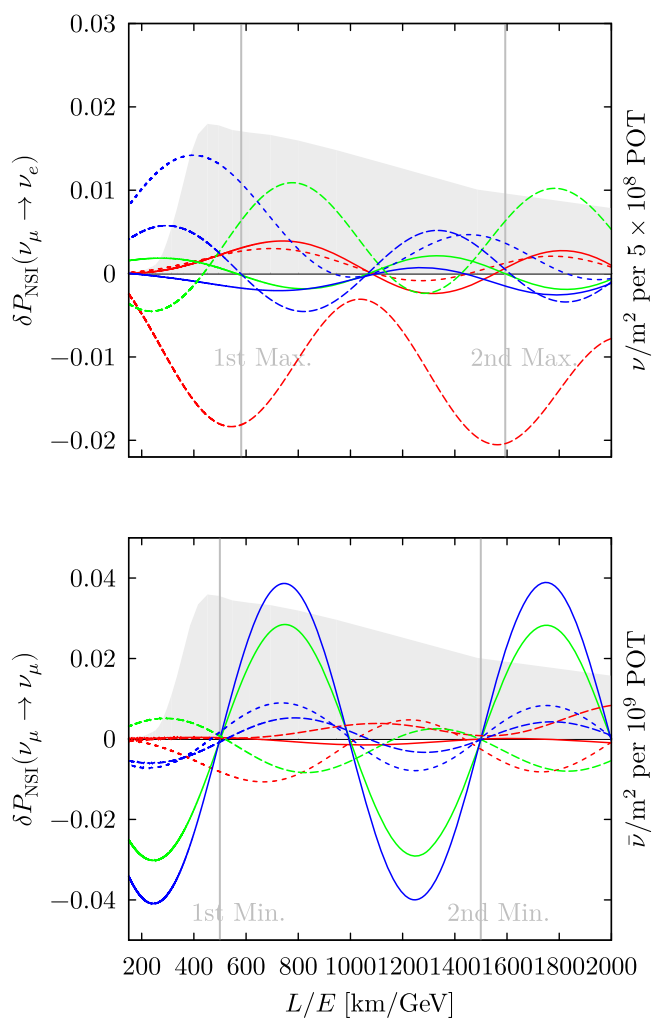

particularly strong particle identification capability as well as good energy resolution, which are both crucial to provide high-efficiency searches and low backgrounds. DUNE covers the first maximum of the appearance channel $(0.5-5 \mathrm{GeV})$, and the wide-band design and LArTPC technology allows it to observe the behavior of $P\left(\nu_{\mu} \rightarrow \nu_{e}\right)$ at energies around the first maximum of the appearance channel with high precision.

We show the difference between oscillation probabilities with one nonzero $\alpha_{m n}$ and those without NSIs, $\delta P_{\mathrm{NSI}}\left(\nu_{\alpha} \rightarrow \nu_{\beta}\right) \equiv P\left(\nu_{\alpha} \rightarrow \nu_{\beta}\right)-P_{0}\left(\nu_{\alpha} \rightarrow \nu_{\beta}\right)$, in Fig. 3. The coefficient $\alpha_{m n}$ is fixed at 0.1 , but the other NSI parameters are fixed at zero. The Dirac phase $\delta=270^{\circ}$ and the normal mass ordering is assumed.

For the appearance channels in the upper two panels of Fig. 3, we see that the NSI parameters nontrivially modify the oscillation probability. NSIs modify the amplitude of the oscillation probability and distort the oscillation behavior against $L / E . \alpha_{23}, \alpha_{31}$, and $\alpha_{33}$ have larger impacts on $\delta P_{\mathrm{NSI}}$ than the other NSI parameters, and $\delta P_{\mathrm{NSI}}$ around the first maximum reaches up to or over 0.01 for the neutrino mode. These impacts are slightly larger in the neutrino mode than in the antineutrino mode, and this is due to our assumption of the normal mass ordering. DUNE's wideband-beam fluxes (grey shaded regions) observe a variation
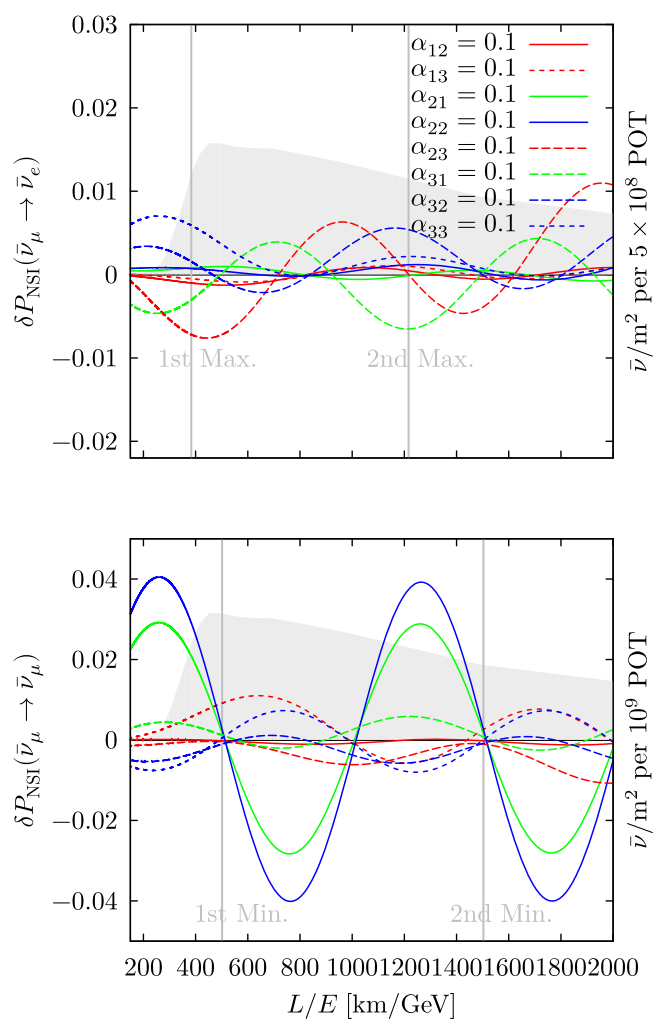

FIG. 3. Oscillation probabilities $\delta P_{\mathrm{NSI}}\left(\nu_{\mu} \rightarrow \nu_{e}\right)$ (upper left), $\delta P_{\mathrm{NSI}}\left(\bar{\nu}_{\mu} \rightarrow \bar{\nu}_{e}\right)$ (upper right), $\delta P_{\mathrm{NSI}}\left(\nu_{\mu} \rightarrow \nu_{\mu}\right)$ (lower left), and $\delta P_{\mathrm{NSI}}\left(\bar{\nu}_{\mu} \rightarrow \bar{\nu}_{\mu}\right)$ (lower right) against $L / E[\mathrm{~km} / \mathrm{GeV}]$ for the case with one $\alpha_{m n}$, fixed at 0.1 . We use the oscillation parameters from the current global fit results [62] (shown in Table IX) for the normal ordering with $\delta=270^{\circ}$, and the oscillation baseline is $1300 \mathrm{~km}$. In the left (right) panels, the grey shaded regions show the $\nu(\bar{\nu})$ flux of the two-horn-optimized design for DUNE at the far detector without oscillations. 
of $\delta P_{\text {NSI }}$ around the first maximum. As a result, the complex behavior in the appearance channel around the first maximum plays the role of distinguishing different textures.

In the lower two panels of Fig. 3, we observe the oscillation behavior of $\delta P_{\mathrm{NSI}}$ in $L / E$ in the disappearance channels, and except for $\alpha_{13}$ it goes to 0 at the first and second minima. As a result, it is clear that we will not see the NSI effects if we focus on the first minimum, which is the approximate location of DUNE's flux peaks. The wideband-beam feature of DUNE (grey shaded regions) provides more information about how much $\alpha_{m n}$ affects the disappearance channels around the first minimum. Further, it is obvious that the disappearance channels can be sensitive to $\alpha_{21}$ and $\alpha_{22}$ as their impacts $\delta P_{\text {NSI }}$ are significantly larger than the others. An interesting feature is that for neutrino and antineutrino modes $\delta P_{\mathrm{NSI}}$ behaves oppositely, i.e., $\delta P_{\mathrm{NSI}}\left(\nu_{\mu} \rightarrow \nu_{\mu}\right) \cong-\delta P_{\mathrm{NSI}}\left(\bar{\nu}_{\mu} \rightarrow \bar{\nu}_{\mu}\right)$. This is because $P\left(\nu_{\mu} \rightarrow \nu_{\mu} ; \delta, A\right) \cong P\left(\bar{\nu}_{\mu} \rightarrow \bar{\nu}_{\mu} ;-\delta,-A\right)$, and also due to the fact that the contribution of $\alpha_{m n}$ is proportional to $A$ in the leading approximation for the disappearance channel. We see this correlation in Fig. 4, in which the event rates with $\alpha_{21}=0.1$ (green curve), $\alpha_{22} \approx 0.7$ (blue circles), and those without NSIs (red curve) are presented in the $\nu$ and $\bar{\nu}$ disappearance channels. The overlap of the blue circles and the green curve demonstrates the difficulty of distinguishing $\alpha_{21}$ and $\alpha_{22}$ in the disappearance channels.

We conclude that the wide-band-beam feature of DUNE is an advantage for detecting NSI textures. Different NSI textures result in different distortions of the probabilities in the appearance channel. Therefore, we can distinguish different textures by reading out the variation of $P\left(\nu_{\mu} \rightarrow \nu_{e}\right)$ with energy. In addition, this feature helps us to measure the size of the NSI effects in the disappearance channel.

\section{B. Testing " $A_{4}$ symmetry" in DUNE}

Matter NSI effects predicted by $A_{4}$-invariant operators only allow diagonal entries. After the breaking of $A_{4}$ by the

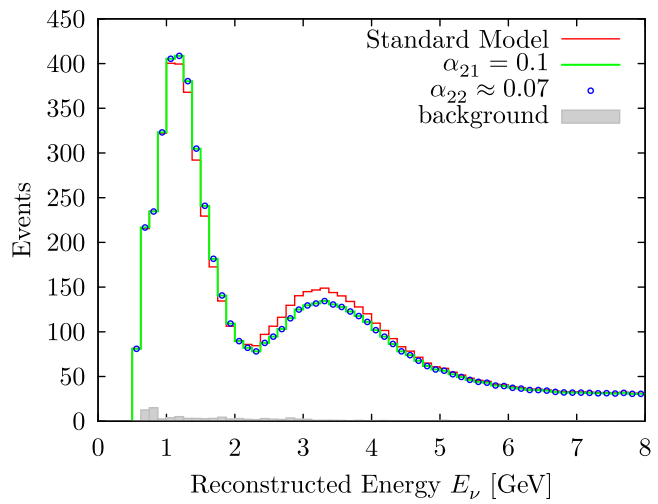

$Z_{2}$-preserving flavon VEV $\chi$, the textures $\mathbb{T}_{2 n}, \mathbb{T}_{3 n}$, or their linear combinations are involved in the NSI matrix $\epsilon$. Equations (D1) and (D2) indicate that accelerator LBL experiments can be sensitive to off-diagonal terms in $\epsilon$, because of the fact that $\epsilon_{\mu \tau}$ is the leading term in the disappearance channel, and $\epsilon_{e \mu}$ and $\epsilon_{e \tau}$ are the leading terms in the appearance channel. As a result, experiments of this kind can test the conservation of $A_{4}$ symmetry.

Throughout this section, we adopt the General Long Baseline Experiment Simulator (GLOBES) library [64,65]. To simulate probabilities with matter-effect NSIs, we modify the default probability engine of GLOBES by simply adding the matrix $A \epsilon$ to the Hamiltonian. For the simulation in DUNE, we implement the simulation package in Ref. [66], with a total run time of 7 years (corresponding to $300 \mathrm{MW} \times$ kton $\times$ years) and a two-horn-optimized beam design with $80 \mathrm{GeV}$ protons. The other sets of oscillation parameters are described in Appendix A.

We study the capacity for DUNE to rule out the " $A_{4}$ symmetry" hypothesis. The statistics quantity that we study is

$$
\left.\Delta \chi_{A_{4}}^{2} \equiv \chi^{2}\right|_{\alpha_{2 n}=\alpha_{3 n}=0}-\chi_{\text {b.f. }}^{2},
$$

where $\left.\chi^{2}\right|_{\alpha_{2 n}=\alpha_{3 n}=0}$ is the $\chi^{2}$ value with the assumption that $\alpha_{2 n}=\alpha_{3 n}=0(n=1,2,3)$, and $\chi_{\text {b.f. }}^{2}$ is the $\chi^{2}$ value for the best fit. The expression for $\chi^{2}$ is

$$
\begin{aligned}
\chi^{2}= & \min _{\Theta, \xi=\left\{\xi_{s}, \xi_{b}\right\}}\left[2 \sum_{i}\left(\eta_{i}(\Theta, \xi)-n_{i}+n_{i} \ln \frac{n_{i}}{\eta_{i}(\Theta, \xi)}\right)\right. \\
& \left.+p(\xi, \sigma)+\mathrm{P}\left(\Theta_{\mathrm{OSC}}\right)\right] .
\end{aligned}
$$

The sum in this expression is over the $i$ energy bins of the experimental configuration, with simulated true event rates $n_{i}$ and simulated event rates $\eta_{i}(\Theta, \xi)$ for the hypothesis parameters $\Theta \equiv\left\{\theta_{i j}, \Delta m_{i j}^{2}\right.$, NSI parameters $\}$ and systematic error parameters $\xi$. Based on different conventions or

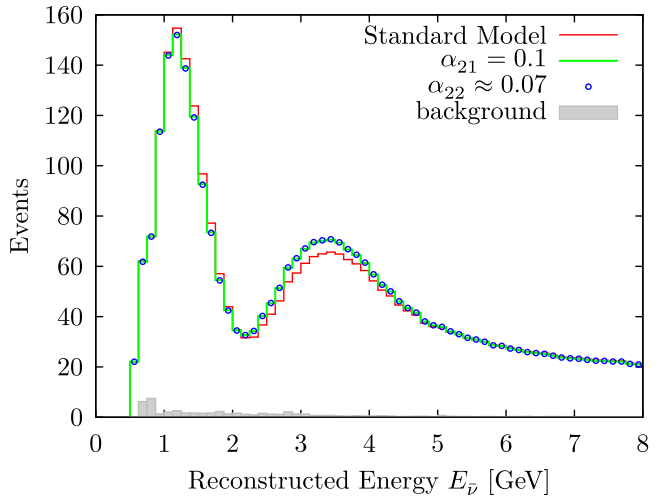

FIG. 4. The event rates with $\alpha_{21}=0.1$ (green curve), $\alpha_{22} \approx 0.07$ (blue circles), and the case without NSIs (red curve). The overlap of the green curve and blue circles represents the correlation between $\alpha_{21}$ and $\alpha_{22}$. 
assumptions, we may adopt different parametrizations for the NSI parameters; in this subsection, we use $\alpha_{m n}$. The systematic errors of the experiments are treated using the method of pulls, parametrized as $\xi_{s}$ for the signal error and $\xi_{b}$ for the background error. These parameters are given Gaussian priors which form the term $p(\xi, \sigma)=$ $\xi_{s}^{2} / \sigma_{s}^{2}+\xi_{b}^{2} / \sigma_{b}^{2}$, where $\sigma=\left\{\sigma_{s}, \sigma_{b}\right\}$ are the sizes of the systematic errors given in Ref. [66]. $\mathrm{P}\left(\Theta_{\mathrm{OSC}}\right)$ comprises a sum of Gaussian priors for the oscillation parameters $\Theta_{\text {OSC., }}$ except for $\delta$. For the central values and widths we use the best-fit and $1 \sigma$ width NuFit results, respectively, which are given in Table IX. The value of $\chi_{\text {b.f. }}^{2}$ is always 0 , as the best fit is exactly the true value. In the following results, we allow $\alpha_{12}$ and $\alpha_{13}$ to vary freely. While varying the true value for one of $\left\{\alpha_{21}, \alpha_{22}, \alpha_{23}, \alpha_{31}, \alpha_{32}, \alpha_{33}\right\}$, we set the true values of $\alpha_{12}$ and $\alpha_{13}$ to be 0 .

We scan all possible true values for the targeted parameter to test the " $A_{4}$ symmetry" hypothesis, i.e., $\alpha_{2 n}=$ $\alpha_{3 n}=0$ (for $n=1,2,3$ ) in Fig. 5. The solid curves and dashed curves correspond to oscillation parameters fixed at their best-fit values and values varying in $1 \sigma$ ranges, as given in Appendix A. The solid (dashed) curves represent the cases with minimum (maximum) correlations with the oscillation parameters. This is for all possible correlations among the parameters. For any flavor model consistent with oscillation data, the $\Delta \chi_{A_{4}}^{2}$ value is located between these two curves. We summarize the above setting in Appendix A 1. The larger $\Delta \chi_{A_{4}}^{2}$ values are seen for $\alpha_{21}$,

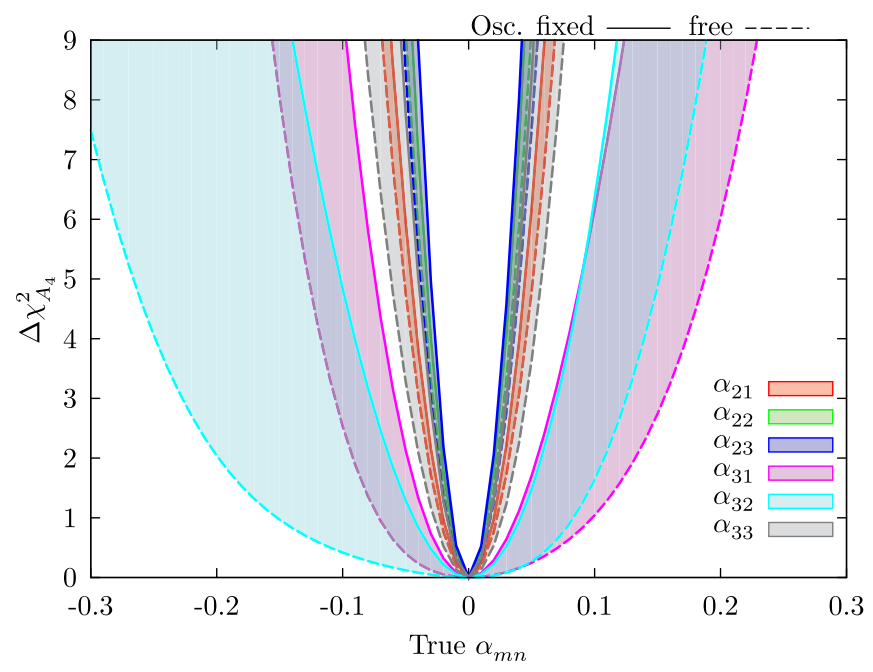

FIG. 5. $\Delta \chi_{A_{4}}^{2}$ to exclude the " $A_{4}$ symmetry" hypothesis $\left(\alpha_{2 n}=\alpha_{3 n}=0\right)$ over the true value from -0.3 to $0.3 . \alpha_{2 n}$ or $\alpha_{3 n}$ are forbidden under the flavor symmetry $A_{4}$. Normal mass ordering with $\delta=270^{\circ}$ is assumed. The solid (dashed) curves represent the fixed (free) oscillation parameters, which can been seen as the cases with the minimum (maximum) correlation with the oscillation parameters. More details about the setting can be seen in Table X. The oscillation parameters are taken from the current global fit results [62] (shown in Table IX). $\alpha_{22}, \alpha_{23}$, and $\alpha_{33}$. For the other two parameters $\alpha_{31}$ and $\alpha_{32}$, which don't perform as well, a minor asymmetry feature is seen. $\alpha_{31}<0$ has a slightly higher significance than $\alpha_{31}>0$. At $\alpha_{31}=0.1$, the exclusion level can reach $1 \leq \Delta \chi_{A_{4}}^{2} \leq 6$; however, at $\alpha_{31}=-0.1, \Delta \chi_{A_{4}}^{2}$ ranges from 2.5 to 9.5 . We see the opposite asymmetry for $\alpha_{32}$, as $1.6 \leq$ $\Delta \chi_{A_{4}}^{2} \leq 6.3\left(0.4 \leq \Delta \chi_{A_{4}}^{2} \leq 4.8\right)$ at $\alpha_{32}=0.1(-0.1)$.

To understand the statistical meaning of the result in Fig. 5, we need to look at Table VI. Given a flavor model that predicts both oscillation and NSI parameters, we should adopt Wilks' theorem. Considering the maximum and minimum of the possible number of degrees of freedom for the $\chi^{2}$ distribution, in Table VI we show the average statistical significance $N \sigma$ to exclude the $A_{4}$ symmetry by simply using Wilks' theorem in the case with a matter effect corresponding to the $1 \sigma$ bounds in Table V. The exclusion level for $\alpha_{23}$ is from $7 \sigma$ to about $10 \sigma$, while that for $\alpha_{21}$ and $\alpha_{22}$ ranges from $\sim 4 \sigma$ to $\sim 6 \sigma$.

We conclude this subsection by noting that DUNE has a high potential to test textures predicted by the " $A_{4}$ symmetry" hypothesis, which only predicts diagonal entries of $\epsilon$.

\section{Testing " $Z_{2}$ symmetry" in DUNE}

From the EFT point of view, combining dimension-eight operators with the $Z_{2}$-preserving flavon VEV can predict plenty of off-diagonal NSI textures. Therefore, testing the " $Z_{2}$ symmetry" by using $Z_{2}$-motivated NSI textures is more complicated than testing the " $A_{4}$ symmetry." Fortunately, some of them have stronger constraints than others if UV completions of these operators are accounted for, and only $\mathbb{T}_{1}, \mathbb{T}_{2}, \mathbb{T}_{3}$, and $\mathbb{T}_{4}$ may reach the percent level, as shown in Sec. IV B. To simplify our discussion, we will only focus on these textures. For clarity, we reparametrize their linear combination as follows:

TABLE VI. The averaged statistical significance to exclude the $A_{4}$ symmetry at the $1 \sigma$ bounds in Table $\mathrm{V}$ for two cases with different degrees of freedom (d.o.f.) using Wills theorem. These two cases are considered to be the maximum and minimum of the possible degrees of freedom. The range is for all possible correlations. The maximum (minimum) number of d.o.f. corresponds to the case with six free oscillation parameters and eight free NSI parameters, compared to the $A_{4}$-symmetry-preserved case with zero (six) free oscillation parameters and two free NSI parameters: $|(6+8)-(0+2)|=12$ for the maximum, while for the minimum $|(6+8)-(6+2)|=6$.

\begin{tabular}{lccc}
\hline \hline \multicolumn{4}{c}{ Parameter } \\
d.o.f. & $\alpha_{21}$ & $\alpha_{22}$ & $\alpha_{23}$ \\
\hline 6 & $4.8 \sigma-5.7 \sigma$ & $4.8 \sigma-5.5 \sigma$ & $7.8 \sigma-10.2 \sigma$ \\
12 & $3.7 \sigma-4.6 \sigma$ & $3.7 \sigma-4.4 \sigma$ & $6.9 \sigma-9.4 \sigma$ \\
\hline \hline
\end{tabular}


TABLE VII. The $1 \sigma$ bounds for $x^{u, d}, y^{u, d}$, and $z^{u, d}$ from the global fit [23] shown in Table IV, and expected $1 \sigma$ bounds on $w$, $x, y$, and $z$ for DUNE with fixed oscillation parameters, assuming true values $w=x=y=z=0$. The superscripts $u$ and $d$ denote NSIs with only $u$ and $d$ quarks, respectively. For both fittings, we allow the other NSI parameters to vary, except for $w$ in the fit using the global fit results. To avoid conflict with the "real $\epsilon_{\alpha \neq \beta}$ " assumption of the global fit, we set $w=0$ in the second and fourth columns.

\begin{tabular}{lclcll}
\hline \hline \multicolumn{1}{l}{ Global Fit } & \multicolumn{2}{l}{ Global Fit } & \multicolumn{2}{l}{ DUNE sensitivity } \\
\hline$w^{u}$ & $\ldots$ & $w^{d}$ & $\ldots$ & $w$ & {$[-0.013,0.025]$} \\
$x^{u}$ & {$[-0.034,0.013]$} & $x^{d}$ & {$[-0.035,0.012]$} & $x$ & {$[-0.1,0.1]$} \\
$y^{u}$ & {$[-0.004,0.003]$} & $y^{d}$ & {$[-0.004,0.003]$} & $y$ & {$[-0.01,0.01]$} \\
$z^{u}$ & {$[-0.002,0.005]$} & $z^{d}$ & {$[-0.002,0.005]$} & $z$ & {$[-0.007,0.017]$} \\
\hline \hline
\end{tabular}

$$
\left(\begin{array}{ccc}
-x & x+y-z-i w & x+y+z+i w \\
x-z+i w & z & y-i w \\
x+z-i w & y+i w & -z
\end{array}\right)
$$

where $x \equiv \alpha_{2}, y \equiv-\frac{\alpha_{1}}{3}+\frac{2 \alpha_{2}}{3 \sqrt{2}}, z \equiv \frac{\alpha_{3}}{\sqrt{3}}$, and $w \equiv \frac{\alpha_{31}}{\sqrt{6}}$. This parametrization applies two strong constraints $\Delta \epsilon_{\mu \tau}$ and $\Delta \tilde{\epsilon}_{\tau \tau}$ to $y$ and $z$, respectively. As we will see later, this helps us to focus on a simple but not highly excluded structure for the NSI matrix.

Table VII shows the $1 \sigma$ constraint on $x, y, z, w$ in Eq. (66) translated from Table IV, and the predicted sensitivity for DUNE with fixed oscillation parameters, assuming $w=x=y=z=0$. For both cases, we test one parameter and allow the others to vary, except for $w$ in the fitting with global fit results. Keeping in mind that $x^{u, d}, y^{u, d}$ and $z^{u, d}$ should be multiplied by a factor $\sim 3$ when comparing with $x, y$, and $z$, we find that the precision for $x, y$, and $z$ for DUNE is competitive with current global fit results. Besides, DUNE is sensitive to the imaginary part $w$, which however is assumed to be zero in the global fit.

We find that the result in Table VII imposes very restrictive bounds on $y$ and $z$ around zeros through the elements $\tilde{\epsilon}_{\tau \tau}$ and $\epsilon_{\mu \tau}$, and the possibility of a nonzero $x$. This result leads to the structure

$$
\epsilon=\left(\begin{array}{lll}
0 & x & x \\
x & x & 0 \\
x & 0 & x
\end{array}\right)
$$

Two sum rules can be read from Eq. (67),

$$
\begin{aligned}
& \epsilon_{e \mu}=\epsilon_{e \tau}=-\tilde{\epsilon}_{e e}, \\
& \epsilon_{\mu \tau}=\tilde{\epsilon}_{\tau \tau}=0 .
\end{aligned}
$$

In the following, we study the exclusion level for DUNE to exclude the matter-effect NSIs in the form of Eq. (67). The statistical quantity that we study is

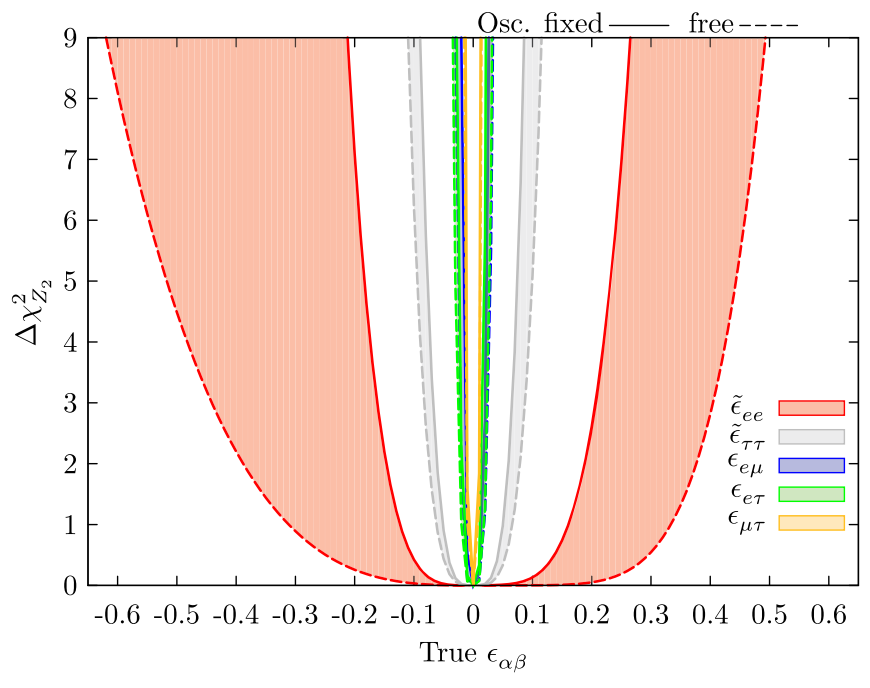

FIG. 6. $\Delta \chi_{Z_{2}}^{2}$ value [defined in Eq. (70)] to exclude the sum rules in Eqs. (68) and (69) over the true value of $-0.65<\epsilon_{\alpha \beta}<0.65$, for normal mass ordering with $\delta=270^{\circ}$. The solid (dashed) curves represent the fixed (free) oscillation parameters, which can been seen as the cases with the minimum (maximum) correlation with the oscillation parameters. Also, we consider all possible numbers of degrees of freedom. In the right panel we show the average statistical significance $N \sigma$ to exclude this model using Wilks' theorem with the $1 \sigma$ bounds in Table IV.

$$
\left.\Delta \chi_{Z_{2}}^{2} \equiv \chi^{2}\right|_{x}-\chi_{\text {b.f. }}^{2}
$$

where $\left.\chi^{2}\right|_{x}$ is the $\chi^{2}$ value defined in Eq. (65), assuming $\varepsilon$ satisfies the structure in Eq. (67). Thus, for $\left.\chi^{2}\right|_{x}$ we use $x$ for the NSI parameters, while for $\chi_{\text {b.f. }}^{2}$, the parametrization $\epsilon_{\alpha \beta}$ is used.

In Fig. 6, we show $\Delta \chi_{Z_{2}}^{2}$ for all possible correlations from $\epsilon_{\alpha \beta}$ or $\epsilon_{\alpha \beta}=-0.65$ to 0.65 . We vary the true value of one certain $\epsilon_{\alpha \beta}$, but fix the others to be zero. We use the

TABLE VIII. The averaged statistical significance to exclude the texture in Eq. (67) for the value of $\tilde{\epsilon}_{\alpha \alpha}$ or $\epsilon_{\alpha \beta}$ corresponding to the $1 \sigma$ bounds in Table IV for two possible numbers of degrees of freedom, approximated by adopting Wilks' theorem. These two cases are considered to be the maximum and minimum of the possible number of degrees of freedom. The range is for all possible correlations. For the number of d.o.f., the maximum (minimum) is the case with six free oscillation parameters and eight free NSI parameters, compared to the hypothetical holding pattern in (67) for NSIs with zero (six) free oscillation parameters and one free NSI parameter: $|(6+8)-(0+1)|=13$ for the maximum, while for the minimum $|(6+8)-(6+1)|=7$.

\begin{tabular}{lccccc}
\hline \hline d.o.f. & $\tilde{\epsilon}_{e e}$ & $\tilde{\epsilon}_{\tau \tau}$ & $\epsilon_{e \mu}$ & $\epsilon_{e \tau}$ & $\epsilon_{\mu \tau}$ \\
\hline 7 & $2.2 \sigma-4.7 \sigma$ & $\sim 0$ & $3.1 \sigma-6.1 \sigma$ & $5.7 \sigma-9.4 \sigma$ & $\sim 0$ \\
13 & $1.1 \sigma-3.7 \sigma$ & $\sim 0$ & $2 \sigma-5.1 \sigma$ & $4.7 \sigma-8.6 \sigma$ & $\sim 0$ \\
\hline \hline
\end{tabular}


same experimental setting and the same oscillation parameter values as in Sec. V B. For the first sum rule, in Eq. (68), within the range $[-0.05,+0.05], \epsilon_{e \mu}$ and $\epsilon_{e \tau}$ can reach a significance $\Delta \chi_{Z_{2}}^{2}>10$. The performance of the ee component is the worst one. For the second sum rule, in Eq. (69), a " $\Delta \chi_{Z_{2}}^{2}<1$ " significance covers roughly $-0.05<\tilde{\epsilon}_{\tau \tau}<0.05$ and $-0.03<\epsilon_{\mu \tau}<0.03$.

As discussed in Sec. VB, we show the statistical significance of every element of the NSI matrix with two possible degrees of freedom, at values of $\tilde{\epsilon}_{\alpha \alpha}$ and $\epsilon_{\alpha \beta}$ corresponding to the $1 \sigma$ bounds in Table IV. These two cases again are for the maximum and minimum of the possible number of degrees of freedom. We find that for the $\tau \tau$ and $\mu \tau$ elements, there is no chance to exclude this model. This is because of the tight constraint on these two elements in the global fit results. We see a high exclusion level for $\epsilon_{e \tau}$; it ranges from $4.7 \sigma$ to $9.4 \sigma$. In the following for $\epsilon_{e \mu}$, the significance is expected to be from $2 \sigma$ to $6.1 \sigma$. For the $e e$ element, we also see a high significance from $1.1 \sigma$ to $4.7 \sigma$.

\section{CONCLUSION}

Non-Abelian discrete flavor symmetries, originally proposed to explain lepton flavor mixing, may contribute to other phenomenological signatures beyond the standard case of third-generation neutrino oscillations. The tests of flavor symmetries have been discussed for a while in the charged lepton sector, but they have not been mentioned in the neutrino sector so far. In this paper, under the assumption of an $A_{4}$ flavor symmetry, we investigated the constraints on matter-effect NSIs imposed by $A_{4}$ symmetry and, after its breaking, those imposed by the residual symmetry $Z_{2}$. We established connections between NSIs and flavor symmetries on two levels: the effective field theory level and the UV completion level.

On the effective field theory level, we imposed $A_{4}$ symmetry on higher-dimensional operators $(d \leq 8)$, which results in NSIs in neutrino oscillations. We only considered operators involving four SM fermions. We have carefully removed those operators that introduce tree-level four-charged-fermion interactions to avoid the strong constraints from the relevant flavor-violating processes. Only one dimension-six operator $\left[\mathcal{O}^{1}=\right.$ $\left.\varepsilon_{a c} \varepsilon_{b d}\left(\overline{L_{a \alpha}} \gamma^{\mu} L_{b \beta}\right)\left(\overline{L_{c \gamma}} \gamma^{\mu} L_{d \delta}\right)\right]$ and seven dimension-eight operators $\quad\left[\mathcal{O}^{2,3,4,5,6}=\left(\overline{\nu_{\alpha \mathrm{L}}} \gamma^{\mu} \nu_{\beta \mathrm{L}}\right)\left(\overline{F_{\gamma}} \gamma_{\mu} F_{\delta}\right) \quad\right.$ (for $\quad F=$ $\left.U_{\mathrm{R}}, D_{\mathrm{R}}, E_{\mathrm{R}}, Q, L\right), \mathcal{O}^{7}=\left(\overline{L_{\alpha}} \tilde{H} \gamma^{\mu} L_{b \beta}\right)\left(\overline{Q_{b \gamma}} \gamma_{\mu} \tilde{H}^{\dagger} Q_{\delta}\right)$, and $\left.\mathcal{O}^{8}=\varepsilon_{b c}\left(\overline{L_{\alpha}} \tilde{H} \gamma^{\mu} L_{b \beta}\right)\left(\overline{Q_{\gamma}} H \gamma_{\mu} Q_{c \delta}\right)\right]$ contribute to mattereffect NSIs, as shown in Table I. Following the general approach used in flavor models, the three lepton doublets $L_{1}, L_{2}$, and $L_{3}$ were arranged as a triplet of $A_{4}$. For any other SM fermions, we performed a scan of all possible representations in the flavor space. By including a flavon with a $Z_{2}$-preserving $\mathrm{VEV}, A_{4}$ is broken to $Z_{2}$, and we obtained $Z_{2}$-motivated NSI textures. Both $A_{4}$-motivated textures and $Z_{2}$-motivated textures have been systematically investigated in this work, with the main result listed in Table II.

Then, we considered how to realize these operators by introducing new particles in renormalizable models of $A_{4}$. The dimension-six operator is realized by introducing electroweak singly charged scalars as mediators. However, this case is strongly suppressed since couplings for $L_{1}, L_{2}$, and $L_{3}$ in $A_{4}$ are correlated with each other, and thus strong constraints from CLFV measurements cannot be avoided. Dimension-eight operators are realized by including heavy sterile neutrinos and charged scalars. The operators $\mathcal{O}^{2,3,4,5}$ involve extra fermions $F=$ $U_{\mathrm{R}}, D_{\mathrm{R}}, E_{\mathrm{R}}, Q$. By arranging $F$ as singlets of $A_{4}$, the couplings for different generation fermions, i.e., $F_{i}$ and $F_{j}$ (for $i \neq j$ ), are not correlated with each other, and the constraints from CLFV measurements or quark-flavorviolating processes do not apply to NSIs. Imposing $A_{4}$ does not give interesting observable NSI textures. After $A_{4}$ is broken to $Z_{2}$, four interesting textures $\mathbb{T}_{1}, \mathbb{T}_{2}, \mathbb{T}_{3}$, and $\mathbb{T}_{4}$, were obtained, as shown in Eq. (50). We refer to them as major textures. The main constraints to these textures are from the measurement of the nonunitary effect of the lepton mixing. Including the experimental constraints, the coefficients of these textures may maximally reach the $10^{-2}$ or $10^{-3}$ level. Arranging $F$ as triplets of $A_{4}$ gives additional NSI textures, all strongly constrained by experiments, and we refer to them as minor textures.

To understand what we can do with NSI textures in the near future, we used the $A_{4}$ - and $Z_{2}$-motivated NSI textures to analyze how to test the flavor symmetry by measuring NSIs in DUNE. We considered all possible correlations and the maximum and minimum numbers of free parameters, which affect the corresponding statistical significance. Two applications were studied. One was a test of " $A_{4}$ symmetry." The off-diagonal entries of the NSI matrix are forbidden by $A_{4}$ symmetry, i.e., $\alpha_{21}=\alpha_{22}=\alpha_{23}=\alpha_{31}=$ $\alpha_{32}=\alpha_{33}=0$. Excluding this hypothesis would allow us to exclude the " $A_{4}$ symmetry," and we predict that DUNE will be able to accomplish this. For the cases with the maximum and minimum numbers of degrees of freedom for the $\chi^{2}$ distribution, in Table VI we show the average statistical significance $N \sigma$ to exclude the $A_{4}$ symmetry using Wilks' theorem in the case with a matter effect corresponding to the $1 \sigma$ bounds in Table V. The exclusion level for $\alpha_{23}$ is from $7 \sigma$ to about $10 \sigma$, while that for $\alpha_{21}$ and $\alpha_{22}$ ranges from $\sim 4 \sigma$ to $\sim 6 \sigma$. High exclusion levels for $\alpha_{3 n}$ $(n=1,2,3)$ are also expected. DUNE can constrain NSI parameters competitively with current global data. In particular, it can measure the imaginary part $w$ with percent precision. We also suggested testing the two sum rules of the NSI parameters, as shown in Eqs. (68) and (69). We showed the statistical significance to exclude the texture in Eq. (67) for every element of the NSI matrix at values 
corresponding to the $1 \sigma$ bounds in Table IV, in the cases with the maximum and minimum numbers of degrees of freedom. We found that, although for the $\tau \tau$ and $\mu \tau$ elements there is no way to exclude this model, the high exclusion level of $\epsilon_{e \tau}$ ranges from $4.7 \sigma$ to $9.4 \sigma$. For $\epsilon_{e \mu}$ and $\tilde{\epsilon}_{e e}$, the significance is expected to be from $2 \sigma$ to $6.1 \sigma$ and $1.1 \sigma$ to $4.7 \sigma$, respectively. We now see good performance for both applications in DUNE.

To summarize, NSIs in neutrino oscillations have been studied in the framework of non-Abelian discrete flavor symmetries for the first time. The textures of NSIs were predicted using flavor symmetries. Measuring these textures can in principle provide a new way to test flavor symmetries and residual symmetries. It is a complimentary to the studies of flavor symmetries in standard neutrino oscillation measurements and CLFV processes. Our simulation results show that even though matter NSI effects are predicted to be small for DUNE in general, these could provide extra information that might extend our understanding of flavor symmetries. And, we showed how useful they are. What we wished to show in this article was not only the theoretical features of flavor symmetries, but also the idea that we cannot waste these small but useful effects. In particular, we note that if $A_{4}$ is conserved at the NSI level, it could be hard to see matter-effect NSIs in DUNE. This is because DUNE is less sensitive to the flavor-conserving effects. Therefore, the null result for the matter-effect NSIs in DUNE could mean that " $A_{4}$ symmetry" is conserved at the NSI level. And this could still extend our knowledge of flavor symmetries at higher energies.

\section{ACKNOWLEDGMENTS}

We thank Silvia Pascoli for very useful discussions and improvements on the manuscript. We are also grateful to Andres Olivares-Del Campo for double checking the probability approximation, Nick Prouse for sharing T2HK simulations, and Jue Zhang for useful discussions. This work has been supported by the European Research Council under ERC Grant NuMass (FP7-IDEAS-ERC ERC-CG 617143), H2020 funded ELUSIVES ITN (H2020-MSCA-ITN-2015, GA-2015-674896-ELUSIVES), InvisiblePlus (H2020MSCA-RISE-2015, GA-2015-690575-InvisiblesPlus), and the Science and Technology Facilities Council (STFC).

\section{APPENDIX A: NEUTRINO OSCILLATION PARAMETERS}

In the standard case, neutrino oscillations are described by the mass-squared differences $\Delta m_{21}^{2}, \Delta m_{31}^{2}$, and $\Delta m_{32}^{2}$, where $\Delta m_{j i}^{2}=m_{j}^{2}-m_{i}^{2}$ and the mixing matrix $U$ is parametrized by three mixing angles $\theta_{i j}$ and a $C P$-violating phase $\delta$ as
TABLE IX. The true values used in this work, unless otherwise stated explicitly, with their uncertainties (the $1 \sigma$ range of the priors we have used in our fit). These are based on NuFit 3.0 (2016) [62]. The definition of $\Delta m_{3 l}^{2}$ is as the same in NuFit 3.0, for normal ordering $\Delta m_{3 l}^{2}=\Delta m_{31}^{2}$, while for the inverse one $\Delta m_{3 l}^{2}=\Delta m_{32}^{2}$.

\begin{tabular}{lcc}
\hline \hline Parameter & Normal ordering & Inverted ordering \\
\hline$\theta_{12}\left[^{\circ}\right]$ & $33.56_{-0.75}^{+0.77}$ & $33.56_{-0.75}^{+0.77}$ \\
$\theta_{13}\left[^{\circ}\right]$ & $8.46_{-0.15}^{+0.15}$ & $8.49_{-0.15}^{+0.15}$ \\
$\theta_{23}\left[^{\circ}\right]$ & $41.6_{-1.2}^{+1.5}$ & $50.0_{-1.4}^{+1.1}$ \\
$\Delta m_{21}^{2}\left[\times 10^{-5} \mathrm{eV}^{2}\right]$ & $7.49_{-0.17}^{+0.19}$ & $7.49_{-0.17}^{+0.17}$ \\
$\Delta m_{3 l}^{2}\left[\times 10^{-3} \mathrm{eV}^{2}\right]$ & $+2.524_{-0.040}^{+0.039}$ & $-2.514_{-0.041}^{+0.038}$ \\
$\delta\left[^{\circ}\right]$ & 270 & 270 \\
\hline \hline
\end{tabular}

$$
\begin{aligned}
U \equiv & \left(\begin{array}{ccc}
1 & 0 & 0 \\
0 & c_{23} & s_{23} \\
0 & -s_{23} & c_{23}
\end{array}\right)\left(\begin{array}{ccc}
c_{13} & 0 & s_{13} \mathrm{e}^{-i \delta} \\
0 & 1 & 0 \\
-s_{13} \mathrm{e}^{i \delta} & 0 & c_{13}
\end{array}\right) \\
& \times\left(\begin{array}{ccc}
c_{12} & s_{13} & 0 \\
-s_{13} & c_{23} & 0 \\
0 & 0 & 1
\end{array}\right)
\end{aligned}
$$

where $s_{i j}=\sin \theta_{i j}$ and $c_{i j}=\cos \theta_{i j}$. Except for $\delta$, we generally adopt the last global fit results in Table IX, taken from Ref. [62], for the true values and the priors. For consistency, we should assume a flavor model for both the oscillation and NSI parameters. However, we do not expect that this will make a large difference since the flavor model should be allowed by global fit results. Further, as the current global result is not significantly changed after including $\mathrm{NO} \nu \mathrm{A}$ data, which may have the impact of NSIs, our results do not lose predictability. Except for $\delta$, we implement priors: we assume Gaussian distributions, centred at the true value with the width taken as the $1 \sigma$ bound from the current global fit results, shown in Table IX.

TABLE X. Summary of the settings for the true and tested values used to study $\Delta \chi_{A_{4}}^{2}$. The oscillation parameters (Osc. Para.) are fixed at the best fit (b.f.) values from the global fit results in Table IX for the true values. We study both scenarios with fixed and varying oscillation parameters with priors, considering all possible correlations. The widths of the priors for the oscillation parameters are the sizes of the $1 \sigma$ uncertainties from the global fit results in Table IX. The flavor symmetry $A_{4}$ only allows $\left\{\alpha_{12}, \alpha_{13}\right\}$, which are fixed at 0 for true values, but are allowed to vary freely for tested values. The parameters $\left\{\alpha_{2 n}\right.$, $\left.\alpha_{3 n}\right\}$ are not allowed by $A_{4}$. For their true values, we study each of them by changing one value from -0.3 to 0.3 , but fixing the other

\begin{tabular}{|c|c|c|c|}
\hline & Osc. Para. & $\alpha_{12}, \alpha_{13}$ & $\alpha_{2 n}, \alpha_{3 n}$ \\
\hline True values & $\begin{array}{l}\text { Fix them } \\
\text { at b.f. }\end{array}$ & Fix them at 0 & $\begin{array}{l}\text { Change one; fix } \\
\text { the other at } 0\end{array}$ \\
\hline Tested values & $\begin{array}{l}\text { All fixed } \\
\text { or free }\end{array}$ & $\begin{array}{l}\text { Allow them } \\
\text { varying }\end{array}$ & Fix all at 0 \\
\hline
\end{tabular}
at 0 . For the tested values, we fix all of them at 0 . 


\section{Parameter settings for the $\boldsymbol{A}_{\mathbf{4}}$ symmetry study}

In Sec. VB we study the potential to exclude the hypothesis of $A_{4}$ symmetry preservation in DUNE. The settings for the oscillation and NSI parameters in the simulation are summarised in Table X.

\section{APPENDIX B: TEXTURES OF NSIS AT THE SOURCE AND DETECTOR PREDICTED BY $A_{4}$}

In this Appendix, we list the textures of NSIs at the source and detector in the framework of $A_{4}$ symmetry. These textures are directly dependent on the fermion representations in the flavor symmetry.

NSIs at the source and detector are expressed as $3 \times 3$ complex matrices $\epsilon^{\mathrm{s}}$ and $\epsilon^{\mathrm{d}}$, respectively, contributing to the superpositions of flavor states,

$$
\begin{aligned}
& \left|\nu_{\alpha}^{\mathrm{s}}\right\rangle=\frac{1}{n_{\alpha}^{\mathrm{s}}}\left(\left|\nu_{\alpha}\right\rangle+\sum_{\beta} \epsilon_{\alpha \beta}^{\mathrm{s}}\left|\nu_{\beta}\right\rangle\right), \\
& \left\langle\nu_{\beta}^{\mathrm{d}}\right|=\frac{1}{n_{\beta}^{\mathrm{d}}}\left(\left\langle\nu_{\beta}\right|+\sum_{\alpha} \epsilon_{\alpha \beta}^{\mathrm{d}}\left\langle\nu_{\alpha}\right|\right),
\end{aligned}
$$

where $n_{\alpha}^{\mathrm{s}}=\sqrt{\sum_{\beta}\left|\delta_{\alpha \beta}+\epsilon_{\alpha \beta}^{\mathrm{s}}\right|^{2}}$ and $n_{\beta}^{\mathrm{d}}=\sqrt{\sum_{\alpha}\left|\delta_{\alpha \beta}+\epsilon_{\alpha \beta}^{\mathrm{d}}\right|^{2}}$ (for $\alpha \neq \beta \neq \gamma \neq \alpha$ ) are normalization factors. Replacing $\varepsilon^{\mathrm{d}, \mathrm{s}}$ with $\varepsilon^{\mathrm{d}, \mathrm{s} *}$, we obtain NSIs for antineutrinos. The effective operators describing NSIs for neutrino production at the source and measured at the detector can be expressed as

$$
\mathcal{L}_{\mathrm{NSI}}=2 \sqrt{2} G_{F} \sum_{p=7}^{12} c_{\alpha \beta \gamma \delta}^{p} \mathcal{O}_{\alpha \beta \gamma \delta}^{p}+\text { H.c. }
$$

Given the higher-dimensional operators in Eq. (19), the relations between the NSI parameters at the source and the detector $\left(\epsilon_{\alpha \beta}^{\mathrm{s}}\right.$ and $\left.\epsilon_{\alpha \beta}^{\mathrm{d}}\right)$ and the higher-dimensional operators are given by

$$
\epsilon_{\alpha \beta}^{\mathrm{s}}=\sum_{p=7}^{12} n^{\mathrm{s}, p} c_{\alpha \beta 11}^{p}, \quad \epsilon_{\alpha \beta}^{\mathrm{d}}=\sum_{p=7}^{12} n^{\mathrm{d}, p} c_{\alpha \beta 11}^{p},
$$

where $n^{\mathrm{s}, p}$ and $n^{\mathrm{d}, p}$ are order-one coefficients related to the number densities of electrons and neutrons.

We only require that the lepton doublets $L=$ $\left(L_{1}, L_{2}, L_{3}\right)^{T}$ be a triplet 3 of $A_{4}(L \mathbf{3})$ to realize large mixing angles, but we do not specify the representations of $A_{4}$ for the rest of the fermions. In other words, they could have any of the following representations:

(1) Three right-handed charged leptons $E_{1 \mathrm{R}}, E_{2 \mathrm{R}}, E_{3 \mathrm{R}}$ are arranged as different singlets of $A_{4}$ or form a triplet 3. The former case is helpful for realizing hierarchical charged lepton masses. Without loss of generality, we consider two cases $\left(E_{\mathrm{R}} \mathbf{1}\right)$ and $\left(E_{\mathrm{R}} \mathbf{3}\right)$ for right-handed charged leptons:

$$
\begin{aligned}
& \left(E_{\mathrm{R}} \mathbf{1}\right) E_{1 \mathrm{R}} \sim \mathbf{1}, E_{2 \mathrm{R}} \sim \mathbf{1}^{\prime}, \quad E_{3 \mathrm{R}} \sim \mathbf{1}^{\prime \prime}, \\
& \left(E_{\mathrm{R}} \mathbf{3}\right) E_{\mathrm{R}}=\left(E_{1 \mathrm{R}}, E_{2 \mathrm{R}}, E_{3 \mathrm{R}}\right) \sim \mathbf{3} .
\end{aligned}
$$

(2) The left-handed quarks $Q_{1}, Q_{2}, Q_{3}$ may also be arranged as different singlets or form a triplet. We consider four cases:

$$
\begin{aligned}
(Q \mathbf{1}) Q_{1} & \sim \mathbf{1}, \\
\left(Q \mathbf{1}^{\prime}\right) Q_{1} & \sim \mathbf{1}^{\prime}, \\
\left(Q \mathbf{1}^{\prime \prime}\right) Q_{1} & \sim \mathbf{1}^{\prime \prime}, \\
(Q \mathbf{3}) \quad Q & =\left(Q_{1}, Q_{2}, Q_{3}\right)^{T} \sim \mathbf{3} .
\end{aligned}
$$

Since $Q_{2}$ and $Q_{3}$ do not contribute to NSIs in neutrino oscillations, we do not care about their representations.

(3) Similarly, we consider two cases for up-type and down-type right-handed quarks, respectively:

$$
\begin{array}{rlrl}
\left(U_{\mathrm{R}} \mathbf{1}\right) U_{1 \mathrm{R}} & \sim \mathbf{1}, & \left(D_{\mathrm{R}} \mathbf{1}\right) D_{1 \mathrm{R}} \sim \mathbf{1}, \\
\left(U_{\mathrm{R}} \mathbf{1}^{\prime}\right) U_{1 \mathrm{R}} & \sim \mathbf{1}^{\prime}, & \left(D_{\mathrm{R}} \mathbf{1}^{\prime}\right) D_{1 \mathrm{R}} \sim \mathbf{1}^{\prime}, \\
\left(U_{\mathrm{R}} \mathbf{1}^{\prime \prime}\right) U_{1 \mathrm{R}} & \sim \mathbf{1}^{\prime \prime}, & \left(D_{\mathrm{R}} \mathbf{1}^{\prime \prime}\right) D_{1 \mathrm{R}} \sim \mathbf{1}^{\prime \prime}, \\
\left(U_{\mathrm{R}} \mathbf{3}\right) U_{\mathrm{R}} & =\left(U_{1 \mathrm{R}}, U_{2 \mathrm{R}}, U_{3 \mathrm{R}}\right)^{T} \sim \mathbf{3}, \\
\left(D_{\mathrm{R}} \mathbf{3}\right) D_{\mathrm{R}} & =\left(D_{1 \mathrm{R}}, D_{2 \mathrm{R}}, D_{3 \mathrm{R}}\right)^{T} \sim \mathbf{3} .
\end{array}
$$

All of the above possibilities are considered in this Appendix.

\section{1. $A_{4}$-invariant operators}

We scan all $A_{4}$-invariant operators $c_{\alpha \beta \gamma \delta}^{7-12} \mathcal{O}_{\alpha \beta \gamma \delta}^{7-12}$, which contribute to NSIs at the source and detector. Besides $\mathbb{T}_{11}$, $\mathbb{T}_{12}$, and $\mathbb{T}_{13}$ in Eq. (22), we find six additional NSI textures:

$$
\begin{aligned}
\mathbb{T}_{11}^{\prime}=\left(\begin{array}{lll}
0 & 1 & 0 \\
0 & 0 & 1 \\
1 & 0 & 0
\end{array}\right), & \mathbb{T}_{12}^{\prime}=\left(\begin{array}{ccc}
0 & -1 & 0 \\
0 & 0 & 2 \\
-1 & 0 & 0
\end{array}\right), \\
\mathbb{T}_{13}^{\prime}=\left(\begin{array}{ccc}
0 & -1 & 0 \\
0 & 0 & 0 \\
1 & 0 & 0
\end{array}\right), & \\
\mathbb{T}_{11}^{\prime \prime}=\left(\begin{array}{lll}
0 & 0 & 1 \\
1 & 0 & 0 \\
0 & 1 & 0
\end{array}\right), & \mathbb{T}_{12}^{\prime \prime}=\left(\begin{array}{ccc}
0 & 0 & -1 \\
-1 & 0 & 0 \\
0 & 2 & 0
\end{array}\right), \\
\mathbb{T}_{13}^{\prime \prime}=\left(\begin{array}{ccc}
-1 & 0 & 0 \\
0 & 0 & 0
\end{array}\right) . &
\end{aligned}
$$


TABLE XI. Operators preserving $A_{4}$ symmetry and the predicted NSI textures at the neutrino source and detector, where $F$ represents any fermion content in the SM and $\mathbf{1}^{0} \equiv \mathbf{1}, D_{i}$ are arbitrary diagonal matrices. Regarding the notation of the representations, for instance, $\left(L \mathbf{3}, E \mathbf{3}, Q \mathbf{1}^{(\prime, \prime \prime)}, U \mathbf{3}\right)$ means $L \sim \mathbf{3}, e \sim \mathbf{3}, Q \sim \mathbf{1}^{(\prime, \prime \prime)}, u \sim \mathbf{3}$, and $D_{\mathrm{R}}$ can take arbitrary representations of $A_{4}$. The textures $\mathbb{T}_{1 n}^{(\prime, \prime)}$ are shown in Eq. (B7).

\begin{tabular}{|c|c|c|c|}
\hline & Representations & $A_{4}$-invariant operators & NSI textures \\
\hline $\mathcal{O}^{7-9}$ & $\begin{array}{l}(L \mathbf{3}) \\
(L \mathbf{3}, F \mathbf{3})\end{array}$ & $\begin{array}{l}(\bar{L} L)_{\mathbf{1}}(\bar{F} F)_{\mathbf{1}} \\
(\bar{L} L)_{\mathbf{3}_{\mathrm{S}}}(\bar{F} F)_{\mathbf{3}_{\mathrm{s}}} \\
(\bar{L} L)_{\mathbf{3}_{\mathrm{A}}}(\bar{F} F)_{\mathbf{3}_{\mathrm{s}}}\end{array}$ & $\begin{array}{l}\mathbb{T}_{11} \\
\mathbb{T}_{12} \\
\mathbb{T}_{13}\end{array}$ \\
\hline \multirow[t]{5}{*}{$\mathcal{O}^{10,12}$} & $\left(L \mathbf{3}, E_{\mathrm{R}} \mathbf{3}, Q \mathbf{3}, U_{\mathrm{R}} \mathbf{3}\right)$ & $\begin{array}{l}\left(\bar{L} E_{\mathrm{R}}\right)_{\mathbf{1}}\left(\bar{Q} U_{\mathrm{R}}\right)_{1} \\
\left(\bar{L} E_{\mathrm{R}}\right)_{\mathbf{3}_{\mathrm{S}}}\left(\bar{Q} U_{\mathrm{R}}\right)_{\mathbf{3}_{\mathrm{S}}} \\
\left(\bar{L} E_{\mathrm{R}}\right)_{\mathbf{3}_{\mathrm{A}}}\left(\bar{Q} U_{\mathrm{R}}\right)_{\mathbf{3}_{\mathrm{S}}}\end{array}$ & $\begin{array}{l}\mathbb{T}_{11} \\
\mathbb{T}_{12} \\
\mathbb{T}_{13}\end{array}$ \\
\hline & $\left(L \mathbf{3}, E_{\mathrm{R}} \mathbf{3}, Q \mathbf{3}, U_{\mathrm{R}} \mathbf{1}^{(\prime, \prime \prime)}\right)$ or $\left(L \mathbf{3}, E_{\mathrm{R}} \mathbf{3}, Q \mathbf{1}^{(\prime \prime \prime \prime)}, U_{\mathrm{R}} \mathbf{3}\right)$ & $\begin{array}{l}\left(\bar{L} E_{\mathrm{R}}\right)_{\mathbf{3}_{\mathrm{S}}}\left(\bar{Q} U_{\mathrm{R}}\right)_{\mathbf{3}} \\
\left(\bar{L} E_{\mathrm{R}}\right)_{\mathbf{3}_{\mathrm{A}}}\left(\bar{Q} U_{\mathrm{R}}\right)_{\mathbf{3}}\end{array}$ & $\begin{array}{l}\mathbb{T}_{12}^{(\prime, \prime \prime)} \\
\mathbb{T}_{13}^{(\prime, \prime \prime)}\end{array}$ \\
\hline & $\begin{array}{l}\left(L \mathbf{3}, E_{\mathrm{R}} \mathbf{3}, Q \mathbf{1}, U_{\mathrm{R}} \mathbf{1}^{(\prime, \prime \prime)}\right),\left(L \mathbf{3}, E_{\mathrm{R}} \mathbf{3}, Q \mathbf{1}^{\prime}, U_{\mathrm{R}} \mathbf{1}^{\prime(\prime \prime, 0)}\right) \\
\quad \text { or }\left(L \mathbf{3}, E_{\mathrm{R}} \mathbf{3}, Q \mathbf{1}^{\prime \prime}, U_{\mathrm{R}} \mathbf{1}^{\prime \prime(0, \prime)}\right)\end{array}$ & $\left(\bar{L} E_{\mathrm{R}}\right)_{\mathbf{1}^{(n, \prime)}}\left(\bar{Q} U_{\mathrm{R}}\right)_{\mathbf{1}^{(,, 1)}}$ & $\mathbb{T}_{11}^{(1, \prime \prime)}$ \\
\hline & $\left(L 3, E_{\mathrm{R}} 1, Q 3, U_{\mathrm{R}} \mathbf{3}\right)$ & $\left(\bar{L} E_{\mathrm{R}}\right)_{\mathbf{3}}\left(\underline{Q} U_{\mathrm{R}}\right)_{\mathbf{3}_{\mathrm{S}}}$ & $D_{1} \mathbb{T}_{11}$ \\
\hline & $\left(L \mathbf{3}, E_{\mathrm{R}} \mathbf{1}, Q \mathbf{1}^{(\prime \prime \prime)}, U_{\mathrm{R}} \mathbf{3}\right)$ or $\left(L \mathbf{3}, E_{\mathrm{R}} \mathbf{1}, Q \mathbf{3}, U_{\mathrm{R}} \mathbf{1}^{(1, \prime \prime)}\right)$ & $\left(\bar{L} E_{\mathrm{R}}\right)_{\mathbf{3}}\left(\bar{Q} U_{\mathrm{R}}\right)_{\mathbf{3}}$ & $D_{2} \mathbb{T}_{11}^{(\prime, \prime \prime)}$ \\
\hline $\mathcal{O}^{11}$ & \multicolumn{3}{|c|}{ Results are obtained from those of $\mathcal{O}^{10,12}$ after the replacements $\bar{Q} \rightarrow \overline{D_{\mathrm{R}}}$ and $U_{\mathrm{R}} \rightarrow Q$. } \\
\hline
\end{tabular}

The operators that may result in these correlations are listed in Table XI.

For $c_{\alpha \beta \gamma \delta}^{7-9} \mathcal{O}_{\alpha \beta \gamma \delta}^{7-9}$, the same discussions on $c_{\alpha \beta \gamma \delta}^{2} \mathcal{O}_{\alpha \beta \gamma \delta}^{2}$ apply to these operators. $c_{\alpha \beta \gamma \delta}^{10-12} \mathcal{O}_{\alpha \beta \gamma \delta}^{10-12}$ provides more textures for NSIs at the source and detector. Here we take $\mathcal{O}_{\alpha \beta \gamma \delta}^{12}$ as an example to obtain these textures in detail.

(1) If $L \sim E_{\mathrm{R}} \sim Q \sim U_{\mathrm{R}} \sim \mathbf{3}$, the $A_{4}$-invariant combinations $\left(\bar{L} E_{\mathrm{R}}\right)_{\mathbf{3}_{\mathrm{S}}}\left(\bar{Q} U_{\mathrm{R}}\right)_{\mathbf{3}_{\mathrm{S}}}$ and $\left(\bar{L} E_{\mathrm{R}}\right)_{\mathbf{3}_{\mathrm{A}}}\left(\bar{Q} U_{\mathrm{R}}\right)_{\mathbf{3}_{\mathrm{S}}}$ result in $\mathbb{T}_{12}$ and $\mathbb{T}_{13}$, respectively.

(2) If $L \sim E_{\mathrm{R}} \sim Q \sim \mathbf{3}$ and $U_{1 \mathrm{R}} \sim \mathbf{1}^{\prime}$, the $A_{4}$-invariant combinations $\left(\bar{L} E_{\mathrm{R}}\right)_{\mathbf{3}_{\mathrm{S}}}\left(\bar{Q} U_{\mathrm{R}}\right)_{\mathbf{3}}$ and $\left(\bar{L} E_{\mathrm{R}}\right)_{\mathbf{3}_{\mathrm{A}}}\left(\bar{Q} U_{\mathrm{R}}\right)_{\mathbf{3}}$ result in $\mathbb{T}_{12}^{\prime}$ and $\mathbb{T}_{13}^{\prime}$, respectively. Replacing $U_{\mathrm{R}} \sim \mathbf{1}^{\prime}$ by $U_{\mathrm{R}} \sim \mathbf{1}^{\prime \prime}$ leads to another two textures, $\mathbb{T}_{12}^{\prime \prime}$ and $\mathbb{T}_{13}^{\prime \prime}$, respectively. These relations are also valid for $L \sim E_{\mathrm{R}} \sim U_{\mathrm{R}} \sim \mathbf{3}, Q \sim \mathbf{1}^{\prime \prime}$, and $\mathbf{1}^{\prime}$, respectively.

(3) If $L \sim E_{\mathrm{R}} \sim \mathbf{3}$ and $Q_{1} \sim U_{1 \mathrm{R}} \sim \mathbf{1}, \mathbf{1}^{\prime}, \mathbf{1}^{\prime \prime}$, the $A_{4^{-}}$ invariant combinations $\left(\bar{L} E_{\mathrm{R}}\right)_{\mathbf{1}}\left(\bar{Q} U_{\mathrm{R}}\right)_{\mathbf{1}}$ result in $\mathbb{T}_{11}$. If $Q_{1}$ and $U_{1 \mathrm{R}}$ belong to different singlets of $A_{4}$, we obtain $\mathbb{T}_{11}^{\prime}$ and $\mathbb{T}_{11}^{\prime \prime}$ for $\overline{Q_{1}} U_{1 \mathrm{R}} \sim \mathbf{1}^{\prime}$ and $\mathbf{1}^{\prime \prime}$, respectively.

(4) If $L \sim Q \sim U_{\mathrm{R}} \sim \mathbf{3}, \quad E_{1 \mathrm{R}} \sim \mathbf{1}, E_{2 \mathrm{R}} \sim \mathbf{1}^{\prime}, E_{3 \mathrm{R}} \sim \mathbf{1}^{\prime \prime}$, we obtain the $A_{4}$-invariant combinations $\sum_{i} y_{i}\left(\bar{L} E_{i \mathrm{R}}\right)_{\mathbf{3}}\left(\bar{Q} U_{\mathrm{R}}\right)_{\mathbf{3}}$ and $\sum_{i} y_{i}^{\prime}\left(\bar{L} E_{i \mathrm{R}}\right)_{\mathbf{3}}\left(\bar{Q} U_{\mathrm{R}}\right)_{\mathbf{3}_{\mathrm{A}}}$, which we denote as $\left(\bar{L} E_{\mathrm{R}}\right)_{\mathbf{3}}\left(\bar{Q} U_{\mathrm{R}}\right)_{\mathbf{3}}$ and $\left(\bar{L} E_{\mathrm{R}}\right)_{\mathbf{3}}\left(\bar{Q} U_{\mathrm{R}}\right)_{\mathbf{3}_{\mathrm{A}}}$, respectively. Here, $y_{i}$ and $y_{i}^{\prime}$ are arbitrary parameters. For the first term we find

$$
c_{\alpha \beta 11}=0 \text { for } \alpha \neq \beta \text {. }
$$

Then, the NSI matrix $\epsilon^{\mathrm{s}, \mathrm{d}}$ can be reexpressed as $D_{1} \mathbb{T}_{11}$, where $D_{1}$ is an arbitrary diagonal matrix. The second operator does not contribute to the NSIs. (5) If $L \sim U_{\mathrm{R}} \sim \mathbf{3}, E_{1 \mathrm{R}} \sim \mathbf{1}, E_{2 \mathrm{R}} \sim \mathbf{1}^{\prime}, E_{3 \mathrm{R}} \sim \mathbf{1}^{\prime \prime}$ and $Q \sim \mathbf{1}$, the $A_{4}$-invariant combinations $\left(\bar{L} E_{\mathrm{R}}\right)_{\mathbf{3}}\left(\bar{Q} U_{\mathrm{R}}\right)_{\mathbf{3}_{\mathrm{S}}}$ only result in an arbitrary diagonal matrix, just like the former item, and we express the NSI matrix $\epsilon^{\mathrm{s}, \mathrm{d}}$ as $D_{2} \mathbb{T}_{11}$, where $D_{2}$ is an arbitrary matrix. Once we change the representation of $Q$ to be $\mathbf{1}^{\prime \prime \prime}(\prime)$, the order of the three components of the triplet $\left(\bar{Q} U_{\mathrm{R}}\right)_{\mathbf{3}_{\mathrm{S}}}$ will be changed, and we arrive at $D_{2} \mathbb{T}_{11}^{\prime(\prime \prime)}$.

Since $\mathcal{O}_{\alpha \beta \gamma \delta}^{10}$ is only different from $\mathcal{O}_{\alpha \beta \gamma \delta}^{12}$ by the Lorentz indices, it gives the same types of correlations as the latter. $\mathcal{O}_{\alpha \beta \gamma \delta}^{11}$ has a different particle arrangement than $\mathcal{O}_{\alpha \beta \gamma \delta}^{12}$. By making the replacements $\bar{Q} \rightarrow \overline{D_{\mathrm{R}}}$ and $Q \rightarrow U_{\mathrm{R}}$, all of the discussions regarding $\mathcal{O}_{\alpha \beta \gamma \delta}^{12}$ apply to $\mathcal{O}_{\alpha \beta \gamma \delta}^{11}$.

The textures in Eq. (B7) only appear at the neutrino source and detector and the NSI matrices $\epsilon^{\mathrm{s}, \mathrm{d}}$ may be combinations of some of $I_{i}, I_{i}^{\prime}$, and $I_{i}^{\prime \prime}$, depending on the choices for the representations of $A_{4}$ to which $E_{\mathrm{R}}, Q, U_{\mathrm{R}}$, and $D_{\mathrm{R}}$ belong. For instance, if $E_{1 \mathrm{R}} \sim \mathbf{1}, E_{2 \mathrm{R}} \sim \mathbf{1}^{\prime}$, $E_{3 \mathrm{R}} \sim \mathbf{1}^{\prime \prime}, Q \sim \mathbf{3}, U_{1 \mathrm{R}} \sim \mathbf{1}, D_{1 \mathrm{R}} \sim \mathbf{1}$, we get the same combination of NSI textures at the source and the detector as in matter,

$$
\epsilon^{\mathrm{s}, \mathrm{d}}=\mathbb{T}_{11} \alpha_{11}^{\mathrm{s}, \mathrm{d}}+\mathbb{T}_{12} \alpha_{12}^{\mathrm{s}, \mathrm{d}}+\mathbb{T}_{13} \alpha_{13}^{\mathrm{s}, \mathrm{d}}
$$

where $\alpha_{1 n}^{\mathrm{s}, \mathrm{d}}$ are complex parameters. Changing the representation of $U_{1 \mathrm{R}}$ to $\mathbf{1}^{\prime}$, we arrive at 


$$
\begin{aligned}
\epsilon^{\mathrm{s}, \mathrm{d}}= & \mathbb{T}_{11} \alpha_{11}^{\mathrm{s}, \mathrm{d}}+\mathbb{T}_{12} \alpha_{12}^{\mathrm{s}, \mathrm{d}}+\mathbb{T}_{13} \alpha_{13}^{\mathrm{s}, \mathrm{d}}+\mathbb{T}_{11}^{\prime} \alpha_{11}^{\mathrm{s}, \mathrm{d}} \\
& +\mathbb{T}_{12} \alpha_{12}^{\mathrm{s}, \mathrm{d}}+\mathbb{T}_{13} \alpha_{13}^{\mathrm{s}, \mathrm{d}},
\end{aligned}
$$

where $\alpha_{1 n}^{(\prime) \mathrm{s}, \mathrm{d}}$ are complex parameters.

\section{2. $Z_{2}$-invariant operators}

Once the operators $\mathcal{O}^{7-12}$ couple to the flavon VEV, $\chi=(1,1,1)^{T} v_{\chi}$, new NSI textures at the source and detector are predicted, as summarized in Table XII. $\chi_{\alpha^{\prime}} \mathcal{O}_{\alpha \beta \gamma \delta \delta}^{7-9}$ give rise to the same textures as in Eq. (33). For $\chi_{\alpha^{\prime}} \mathcal{O}_{\alpha \beta \gamma \delta}^{10-12}$, we follow the same procedure as in the last section, taking $\chi_{\alpha^{\prime}} \mathcal{O}_{\alpha \beta \gamma \delta}^{12}$ as an example:

(1) If $L \sim E_{\mathrm{R}} \sim Q \sim U_{\mathrm{R}} \sim \mathbf{3}$, the $Z_{2}$-invariant operators $\chi\left(\left(\bar{L} E_{\mathrm{R}}\right)_{\mathbf{3}_{\mathrm{S}}}\left(\bar{Q} U_{\mathrm{R}}\right)_{\mathbf{3}_{\mathrm{S}}}\right)_{\mathbf{3}_{\mathrm{S}}}, \chi\left(\left(\bar{L} E_{\mathrm{R}}\right)_{\mathbf{3}_{\mathrm{A}}}\left(\bar{Q} U_{\mathrm{R}}\right)_{\mathbf{3}_{\mathrm{S}}}\right)_{\mathbf{3}_{\mathrm{S}}}$, $\chi\left(\left(\bar{L} E_{\mathrm{R}}\right)_{\mathbf{3}_{\mathrm{S}}}\left(\bar{Q} U_{\mathrm{R}}\right)_{\mathbf{3}_{\mathrm{S}}}\right)_{\mathbf{3}_{\mathrm{A}}}$, and $\chi\left(\left(\bar{L} E_{\mathrm{R}}\right)_{\mathbf{3}_{\mathrm{A}}}\left(\bar{Q} U_{\mathrm{R}}\right)_{\mathbf{3}_{\mathrm{S}}}\right)_{\mathbf{3}_{\mathrm{A}}}$ result in the textures $3 \mathbb{T}_{12}-\mathbb{T}_{22}, 3 \mathbb{T}_{13}+\mathbb{T}_{23}, \mathbb{T}_{32}$, and $\mathbb{T}_{33}$, respectively. By changing the representations to $L \sim E_{\mathrm{R}} \sim Q \sim \mathbf{3}$ and $U_{1 \mathrm{R}} \sim \mathbf{1}^{(1, \prime \prime)}$, or $L \sim$ $E_{\mathrm{R}} \sim U_{\mathrm{R}} \sim \mathbf{3}$ and $Q_{1} \sim \mathbf{1}^{(1, \prime \prime)}$, we arrive at the same textures.
(2) If $L \sim E_{\mathrm{R}} \sim \mathbf{3}$ and $Q_{1}, U_{1 \mathrm{R}} \sim \mathbf{1}, \mathbf{1}^{\prime}, \mathbf{1}^{\prime \prime}$, the $Z_{2^{-}}$ invariant combinations $\chi\left(\left(\bar{L} E_{\mathrm{R}}\right)_{\mathbf{3}_{\mathrm{S}}}\left(\bar{Q} U_{\mathrm{R}}\right)_{\mathbf{1}, \mathbf{1}^{\prime}, \mathbf{1}^{\prime \prime}}\right)_{\mathbf{3}}$, $\chi\left(\left(\bar{L} E_{\mathrm{R}}\right)_{\mathbf{3}_{\mathrm{A}}}\left(\bar{Q} U_{\mathrm{R}}\right)_{\mathbf{1}_{1} \mathbf{1}^{\prime}, \mathbf{1}^{\prime \prime}}\right)_{\mathbf{3}}$ result in $\mathbb{T}_{21}$ and $\mathbb{T}_{22}$, respectively.

(3) If $L \sim Q \sim U_{\mathrm{R}} \sim \mathbf{3}, E_{1 \mathrm{R}} \sim \mathbf{1}, E_{2 \mathrm{R}} \sim \mathbf{1}^{\prime}, E_{3 \mathrm{R}} \sim \mathbf{1}^{\prime \prime}$, the operator $\sum_{i} y_{i}^{\prime \prime} \chi\left(\bar{L} E_{i \mathrm{R}}\right)_{\mathbf{3}}\left(\bar{Q} U_{\mathrm{R}}\right)_{\mathbf{1}}$ requires

$$
\begin{array}{ll}
c_{e e 11}=c_{e \mu 11}=c_{e \tau 11}, & c_{\mu e 11}=c_{\mu \mu 11}=c_{\mu \tau 11}, \\
c_{\tau e 11}=c_{\tau \mu 11}=c_{\tau \tau 11}, &
\end{array}
$$

where there is no correlation between $c_{\alpha \beta 11}$ and $c_{\alpha^{\prime} \beta^{\prime} 11}$ once $\alpha \neq \alpha^{\prime}$. It gives rise to the NSI texture

$$
\left(\begin{array}{lll}
y_{1}^{\prime \prime} & y_{1}^{\prime \prime} & y_{1}^{\prime \prime} \\
y_{2}^{\prime \prime} & y_{2}^{\prime \prime} & y_{2}^{\prime \prime} \\
y_{3}^{\prime \prime} & y_{3}^{\prime \prime} & y_{3}^{\prime \prime}
\end{array}\right)=\left(\begin{array}{ccc}
y_{1}^{\prime \prime} & 0 & 0 \\
0 & y_{2}^{\prime \prime} & 0 \\
0 & 0 & y_{3}^{\prime \prime}
\end{array}\right) \mathbb{T}_{1}^{\prime} \mathbb{T}_{11},
$$

\begin{tabular}{|c|c|c|c|}
\hline & Representations & $Z_{2}$-invariant operators & NSI textures \\
\hline \multirow[t]{2}{*}{$\chi \mathcal{O}^{7-9}$} & $(L \mathbf{3})$ & $\begin{array}{l}\chi(\bar{L} L)_{\mathbf{3}_{\mathrm{S}}}(\bar{F} F)_{\mathbf{1}} \\
\chi(\bar{L} L)_{\mathbf{3}_{\mathrm{A}}}(\bar{F} F)_{\mathbf{1}}\end{array}$ & $\begin{array}{l}\mathbb{T}_{12}+\mathbb{T}_{22} \\
\mathbb{T}_{13}+\mathbb{T}_{23}\end{array}$ \\
\hline & $(L \mathbf{3}, F \mathbf{3})$ & $\begin{array}{l}\chi\left((\bar{L} L)_{\mathbf{3}_{\mathrm{S}}}(\bar{F} F)_{\mathbf{3}_{\mathrm{S}}}\right)_{\mathbf{3}_{\mathrm{S}}} \\
\chi\left((\bar{L} L)_{\mathbf{3}_{\mathrm{A}}}(\bar{F} F)_{\mathbf{3}_{\mathrm{s}}}\right)_{\mathbf{3}_{\mathrm{S}}} \\
\chi\left((\bar{L} L)_{\mathbf{3}_{\mathrm{S}}}(\bar{F} F)_{\mathbf{3}_{\mathrm{s}}}\right)_{\mathbf{3}_{\mathrm{A}}} \\
\chi\left((\bar{L} L)_{\mathbf{3}_{\mathrm{A}}}(\bar{F} F)_{\mathbf{3}_{\mathrm{s}}}\right)_{\mathbf{3}_{\mathrm{A}}}\end{array}$ & $\begin{array}{c}2 \mathbb{T}_{12}-\mathbb{T}_{22} \\
2 \mathbb{T}_{13}-\mathbb{T}_{23} \\
\mathbb{T}_{32} \\
\mathbb{T}_{33}\end{array}$ \\
\hline \multirow[t]{4}{*}{$\chi \mathcal{O}^{10,12}$} & $\left(L \mathbf{3}, E_{\mathrm{R}} \mathbf{3}, Q \mathbf{3}, U_{\mathrm{R}} \mathbf{3}\right)$ & 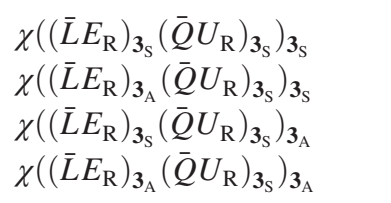 & $\begin{array}{c}2 \mathbb{T}_{12}-\mathbb{T}_{22} \\
2 \mathbb{T}_{13}-\mathbb{T}_{23} \\
\mathbb{T}_{32} \\
\mathbb{T}_{33}\end{array}$ \\
\hline & $\left(L \mathbf{3}, E_{\mathrm{R}} \mathbf{3}, Q \mathbf{3}, U_{\mathrm{R}} \mathbf{1}^{(\prime, \prime \prime)}\right)$ or $\left(L \mathbf{3}, E_{\mathrm{R}} \mathbf{3}, Q \mathbf{1}^{(\prime, \prime \prime)}, U_{\mathrm{R}} \mathbf{3}\right)$ & $\begin{array}{l}\chi\left(\left(\bar{L} E_{\mathrm{R}}\right)_{\mathbf{3}_{\mathrm{S}}}\left(\bar{Q} U_{\mathrm{R}}\right)_{\mathbf{3}}\right)_{\mathbf{3}_{\mathrm{S}}} \\
\chi\left(\left(\bar{L} E_{\mathrm{R}}\right)_{\mathbf{3}_{\mathrm{A}}}\left(\bar{Q} U_{\mathrm{R}}\right)_{\mathbf{3}}\right)_{\mathbf{3}_{\mathrm{S}}} \\
\chi\left(\left(\bar{L} E_{\mathrm{R}}\right)_{\mathbf{3}_{\mathrm{S}}}\left(\bar{Q} U_{\mathrm{R}}\right)_{\mathbf{3}}\right)_{\mathbf{3}_{\mathrm{A}}} \\
\chi\left(\left(\bar{L} E_{\mathrm{R}}\right)_{\mathbf{3}_{\mathrm{A}}}\left(\bar{Q} U_{\mathrm{R}}\right)_{\mathbf{3}}\right)_{\mathbf{3}_{\mathrm{A}}}\end{array}$ & $\begin{array}{c}2 \mathbb{T}_{12}-\mathbb{T}_{22} \\
2 \mathbb{T}_{13}-\mathbb{T}_{23} \\
\mathbb{T}_{32} \\
\mathbb{T}_{33}\end{array}$ \\
\hline & $\begin{array}{l}\left(L \mathbf{3}, E_{\mathrm{R}} \mathbf{3}, Q \mathbf{1}, U_{\mathrm{R}} \mathbf{1}^{(\prime, \prime \prime)}\right),\left(L \mathbf{3}, E_{\mathrm{R}} \mathbf{3}, Q \mathbf{1}^{\prime}, U_{\mathrm{R}} \mathbf{1}^{\prime(\prime \prime, 0)}\right) \\
\quad \text { or }\left(L \mathbf{3}, E_{\mathrm{R}} \mathbf{3}, Q \mathbf{1}^{\prime \prime}, U_{\mathrm{R}} \mathbf{1}^{\prime \prime(0, \prime)}\right)\end{array}$ & 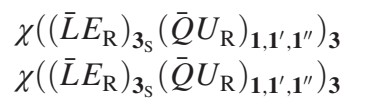 & $\begin{array}{l}\mathbb{T}_{12}+\mathbb{T}_{22} \\
\mathbb{T}_{13}+\mathbb{T}_{23}\end{array}$ \\
\hline & $\left(L \mathbf{3}, E_{\mathrm{R}} \mathbf{1}, Q \mathbf{1}^{((, \prime)}, U_{\mathrm{R}} \mathbf{3}\right)$ or $\left(L \mathbf{3}, E_{\mathrm{R}} \mathbf{1}, Q \mathbf{3}, U_{\mathrm{R}} \mathbf{1}^{(1, \prime \prime)}\right)$ & $\begin{array}{l}\chi\left(\bar{L} E_{\mathrm{R}}\right)_{\mathbf{3}}\left(\bar{Q} U_{\mathrm{R}}\right)_{\mathbf{1}} \\
\chi\left(\left(\bar{L} E_{\mathrm{R}}\right)_{\mathbf{3}}\left(\bar{Q} U_{\mathrm{R}}\right)_{\mathbf{3}_{\mathrm{s}}}\right)_{\mathbf{3}_{\mathrm{S}}} \\
\chi\left(\left(\bar{L} E_{\mathrm{R}}\right)_{\mathbf{3}}\left(\bar{Q} U_{\mathrm{R}}\right)_{\mathbf{3}_{\mathrm{S}}}\right)_{\mathbf{3}_{\mathrm{A}}} \\
\chi\left(\left(\bar{L} E_{\mathrm{R}}\right)_{\mathbf{3}}\left(\bar{Q} U_{\mathrm{R}}\right)_{\mathbf{3}}\right)_{\mathbf{3}_{\mathrm{S}}} \\
\chi\left(\left(\bar{L} E_{\mathrm{R}}\right)_{\mathbf{3}}\left(\bar{Q} U_{\mathrm{R}}\right)_{3}\right)_{\mathbf{3}_{\mathrm{A}}}\end{array}$ & $\begin{array}{l}D_{3} \mathbb{T}_{1}^{\prime} \mathbb{T}_{11} \\
D_{4} \mathbb{T}_{1}^{\prime} \mathbb{T}_{12} \\
D_{5} \mathbb{T}_{1}^{\prime} \mathbb{T}_{13} \\
D_{6} \mathbb{T}_{1}^{\prime} \mathbb{T}_{12} \\
D_{7} \mathbb{T}_{1}^{\prime} \mathbb{T}_{13}\end{array}$ \\
\hline$\chi \mathcal{O}^{11}$ & \multicolumn{3}{|c|}{ Results are obtained from those of $\chi \mathcal{O}^{10,12}$ after the replacements $\bar{Q} \rightarrow \overline{D_{\mathrm{R}}}$ and $U_{\mathrm{R}} \rightarrow Q$. } \\
\hline
\end{tabular}

where

TABLE XII. Operators preserving the residual symmetry $Z_{2}, Z_{2} \subset A_{4}$, and the resulting NSI textures at the neutrino source and detector, where $F$ represents any fermion content in the SM. The NSI parameter correlations $\mathbb{T}_{2 n}$ and $\mathbb{T}_{3 n}$ are shown in Eq. (33). $D_{i}$ are arbitrary diagonal matrices. 


$$
\begin{aligned}
& \mathbb{T}_{1}^{\prime}=\left(\begin{array}{lll}
1 & 1 & 1 \\
1 & 1 & 1 \\
1 & 1 & 1
\end{array}\right) . \\
& \chi\left(\left(\bar{L} E_{\mathrm{R}}\right)_{\mathbf{3}}\left(\bar{Q} U_{\mathrm{R}}\right)_{\mathbf{3}_{\mathrm{S}}}\right)_{\mathbf{3}_{\mathrm{S}}} \quad \text { and } \quad \chi\left(\left(\bar{L} E_{\mathrm{R}}\right)_{\mathbf{3}}\left(\bar{Q} U_{\mathrm{R}}\right)_{\mathbf{3}_{\mathrm{S}}}\right)_{\mathbf{3}_{\mathrm{A}}}
\end{aligned}
$$
lead to

$c_{e e 11}=-2 c_{e \mu 11}=-2 c_{e \tau 11}$,

$c_{\mu e 11}=-2 c_{\mu \mu 11}=-2 c_{\mu \tau 11}$,

$c_{\tau e 11}=-2 c_{\tau \mu 11}=-2 c_{\tau \tau 11}$;

$c_{e \mu 11}=-c_{e \tau 11}, \quad c_{\mu \mu 11}=-c_{\mu \tau 11}$,

$c_{\tau \mu 11}=-c_{\tau \tau 11}$,

respectively, and there is no correlation between $c_{\alpha \beta 11}$ and $c_{\alpha^{\prime} \beta^{\prime} 11}$ for $\alpha \neq \alpha^{\prime}$ in each case. From these two operators, we obtain the NSI textures

$$
D_{4} \mathbb{T}_{1}^{\prime} \mathbb{T}_{12}, \quad D_{5} \mathbb{\varpi}_{1}^{\prime} \mathbb{T}_{13},
$$

respectively, where $D_{i}$ are independently arbitrary diagonal matrices. Replacing the representation of $Q$ by any singlet $\mathbf{1}, \mathbf{1}^{\prime}$, or $\mathbf{1}^{\prime \prime}$, we obtain the $Z_{2}$-invariant operators $\chi\left(\left(\bar{L} E_{\mathrm{R}}\right)_{\mathbf{3}}\left(\bar{Q} U_{\mathrm{R}}\right)_{\mathbf{3}}\right)_{\mathbf{3}_{\mathrm{S}}}$ and $\chi\left(\left(\bar{L} E_{\mathrm{R}}\right)_{\mathbf{3}}\left(\bar{Q} U_{\mathrm{R}}\right)_{\mathbf{3}}\right)_{\mathbf{3}_{\mathrm{A}}}$, which give the similar textures $D_{6} \mathbb{T}_{1}^{\prime} \mathbb{T}_{12}$ and $D_{7} \mathbb{T}_{1}^{\prime} \mathbb{T}_{13}$, respectively, with $D_{6}$ and $D_{7}$ being arbitrary diagonal matrices.

\section{APPENDIX C: MATHEMATICAL PROPERTIES OF $\mathbb{T}_{i}$}

The textures $\mathbb{T}_{i}$ satisfy the following interesting mathematical properties. They are helpful for our discussion in Sec. IV.

(1) $\mathbb{T}_{i}$ (for $i=1,2,3,4$ ) form the following "closed" algebras:

$$
\begin{aligned}
\mathbb{T}_{i}^{2} & =\mathbb{T}_{1}, \quad \mathbb{T}_{1} \mathbb{\mathbb { T }}_{i}=\mathbb{\mathbb { T }}_{i}, \quad \mathbb{T}_{2} \mathbb{\mathbb { T }}_{3}=-i \mathbb{\mathbb { T }}_{4}, \\
\mathbb{T}_{2} \mathbb{\mathbb { T }}_{4} & =i \mathbb{\mathbb { T }}_{3}, \quad \mathbb{T}_{3} \mathbb{\mathbb { T }}_{4}=-i \mathbb{\mathbb { T }}_{2} .
\end{aligned}
$$

(2) Given two $3 \times 3$ coupling matrices or mass matrices $M_{1}=\alpha_{0} \mathbb{1}+\sum_{i=1}^{4} \alpha_{i} \mathbb{\mathbb { T }}_{i}$ and $M_{2}=\beta_{0} \mathbb{1}+\sum_{i=1}^{4} \beta_{i} \mathbb{\mathbb { T }}_{i}$, their product $M_{1} M_{2}$ is a linear combination of 1 and $\mathbb{T}_{i}$,

$$
\begin{aligned}
M_{1} M_{2}= & \alpha_{0} \beta_{0} \mathbb{1}+\left(\alpha_{0} \beta_{1}+\alpha_{1} \beta_{0}+\alpha_{1} \beta_{1}+\alpha_{2} \beta_{2}+\alpha_{3} \beta_{3}+\alpha_{4} \beta_{4}\right) \mathbb{T}_{1} \\
& +\left(\alpha_{0} \beta_{2}+\alpha_{2} \beta_{0}+\alpha_{1} \beta_{2}+\alpha_{2} \beta_{1}+i \alpha_{4} \beta_{3}-i \alpha_{3} \beta_{4}\right) \mathbb{T}_{2} \\
& +\left(\alpha_{0} \beta_{3}+\alpha_{3} \beta_{0}+\alpha_{1} \beta_{3}+\alpha_{3} \beta_{1}+i \alpha_{2} \beta_{4}-i \alpha_{4} \beta_{2}\right) \mathbb{T}_{3} \\
& +\left(\alpha_{0} \beta_{4}+\alpha_{4} \beta_{0}+\alpha_{1} \beta_{4}+\alpha_{4} \beta_{1}+i \alpha_{3} \beta_{2}-i \alpha_{2} \beta_{3}\right) \mathbb{T}_{4} .
\end{aligned}
$$

(3) If $M_{1}$ is reversible, the inverse matrix $M_{1}^{-1}$

$$
\begin{aligned}
& M_{1}^{-1}=\frac{\alpha_{0}}{\operatorname{det} A}\left[\frac{\operatorname{det} A}{\alpha_{0}^{2}} \mathbb{1}+\left(\alpha_{0}+\alpha_{1}-\frac{\operatorname{det} A}{\alpha_{0}^{2}}\right) \mathbb{\mathbb { T }}_{1}\right. \\
& \left.-\alpha_{2} \mathbb{T}_{2}-\alpha_{3} \mathbb{T}_{3}-\alpha_{4} \mathbb{T}_{4}\right] \text {, }
\end{aligned}
$$

where $\operatorname{det} M_{1}=\alpha_{0}\left(\alpha_{0}^{2}+2 \alpha_{0} \alpha_{1}+\alpha_{1}^{2}-\alpha_{2}^{2}-\alpha_{3}^{2}-\alpha_{4}^{2}\right)$ is also a linear combination of 1 and $\mathbb{T}_{i}$.

By setting some of $\alpha_{i}$ or $\beta_{i}$ to zero, the following corollaries are obtained:

(1) $\mathbb{1}$ and $\mathbb{T}_{1}$ form a closed algebra: if $M_{1}, M_{2}$ are linear combinations of 1 and $\mathbb{T}_{1}$, their product and inverse matrices (if reversible) are also linear combinations of 1 and $\mathbb{T}_{1}$.

(2) $\mathbb{1}, \mathbb{T}_{1}$, and $\mathbb{T}_{2}$ form a closed algebra: if $M_{1}, M_{2}$ are linear combinations of $\mathbb{1}, \mathbb{T}_{1}$, and $\mathbb{T}_{2}$, their product and inverse matrices (if reversible) are also linear combinations of $\mathbb{1}, \mathbb{T}_{1}$, and $\mathbb{T}_{2}$.

(3) $\mathbb{1}, \mathbb{T}_{1}$, and $\mathbb{T}_{3}$ form a closed algebra: if $M_{1}, M_{2}$ are linear combinations of $\mathbb{1}, \mathbb{T}_{1}$, and $\mathbb{T}_{2}$, their product and inverse matrices (if reversible) are also linear combinations of $\mathbb{1}, \mathbb{T}_{1}$, and $\mathbb{T}_{3}$.

\section{APPENDIX D: OSCILLATION PROBABILITIES WITH MATTER-EFFECT NSIS}

To understand the impact of $\alpha_{m n}^{\mathrm{m}}$ (in the following, we simply use $\alpha_{m n}$ ) on neutrino oscillation probabilities, we consider the probabilities with nonzero $\epsilon_{\alpha \beta}^{\mathrm{m}}$ (in the following, we simply use $\epsilon_{\alpha \beta}$ ). Therefore, we first study the probability including the NSI matter effects in terms of $\epsilon_{\alpha \beta}$, and then, by using the relations between the two parameter sets in Table III, we can extend our understanding of how the flavor symmetry model realizes the oscillation probability through matter-effect NSIs.

Assuming $\sqrt{\frac{\Delta m_{21}^{2}}{\Delta m_{31}^{2}}} \sim \sqrt{\left|\epsilon_{\alpha \beta}\right|} \sim s_{13}$ as the first-order perturbation terms $\xi$, we expand the disappearance oscillation probability $P\left(\nu_{\mu} \rightarrow \nu_{\mu}\right)$ and appearance oscillation probability $P\left(\nu_{\mu} \rightarrow \nu_{e}\right)$. These equations are given with the leading-order coefficient for each $\epsilon_{\alpha \beta}$ to understand how each element affects the probability at the leading order: ${ }^{7}$

\footnotetext{
${ }^{7}$ Our results are consistent with those of Ref. [67].
} 


$$
\begin{aligned}
P\left(\nu_{\mu} \rightarrow \nu_{\mu}\right)= & P_{0}\left(\nu_{\mu} \rightarrow \nu_{\mu}\right)+\delta P_{\mathrm{NSI}}\left(\nu_{\mu} \rightarrow \nu_{\mu}\right) \\
\approx & P_{0}\left(\nu_{\mu} \rightarrow \nu_{\mu}\right)-A \epsilon_{\mu \tau} \cos \phi_{\mu \tau}\left(\sin ^{3} 2 \theta_{23} \frac{L}{2 E} \sin 2 \Delta_{31} L+4 \sin 2 \theta_{23} \cos ^{2} 2 \theta_{23} \frac{1}{\Delta m_{31}^{2}} \sin ^{2} \Delta_{31} L\right) \\
& -A \tilde{\epsilon}_{\tau \tau} c_{23}^{2} s_{23}^{2}\left(c_{23}^{2}-s_{23}^{2}\right)\left(\frac{L}{8 E} \sin 2 \Delta_{31} L-\frac{1}{\Delta m_{31}^{2}} \sin ^{2} \Delta_{31} L\right) \\
& +\mathcal{C}_{\mu \rightarrow e ; e \mu}^{1}\left|\epsilon_{e \mu}\right|+\mathcal{C}_{\mu \rightarrow e ; e \tau}^{1}\left|\epsilon_{e \tau}\right|+\mathcal{C}_{\mu \rightarrow e ; e e}^{2} \tilde{\epsilon}_{e e},
\end{aligned}
$$

$$
\begin{aligned}
P\left(\nu_{\mu} \rightarrow \nu_{e}\right)= & P_{0}\left(\nu_{\mu} \rightarrow \nu_{e}\right)+\delta P_{\mathrm{NSI}}\left(\nu_{\mu} \rightarrow \nu_{e}\right) \\
\approx & P_{0}\left(\nu_{\mu} \rightarrow \nu_{e}\right)+8 s_{13}\left|\epsilon_{e \mu}\right| s_{23} \frac{\Delta m_{31}^{2}}{\Delta m_{31}^{2}-A} \sin \Delta_{31}^{A} L \\
& \times\left(s_{23}^{2} \frac{A}{\Delta m_{31}^{2}-A} \cos \left(\delta+\phi_{e \mu}\right) \sin \Delta_{31}^{A} L+c_{23}^{2} \sin \frac{A L}{4 E} \cos \left(\delta+\phi_{e \mu}-\Delta_{31} L\right)\right) \\
& +8 s_{13}\left|\epsilon_{e \tau}\right| c_{23} s_{23}^{2} \frac{\Delta m_{31}^{2}}{\Delta m_{31}^{2}-A} \sin \Delta_{31}^{A} L \\
& \times\left(\frac{A}{\Delta m_{31}^{2}-A} \cos \left(\delta+\phi_{e \tau}\right) \sin \Delta_{31}^{A} L-\sin \frac{A L}{4 E} \cos \left(\delta+\phi_{e \tau}-\Delta_{31} L\right)\right) \\
& +\mathcal{C}_{\mu \rightarrow e ; \mu \tau}^{2}\left|\epsilon_{\mu \tau}\right|+\mathcal{C}_{\mu \rightarrow e ; e e}^{2} \tilde{\epsilon}_{e e}+\mathcal{C}_{\mu \rightarrow e ; \tau \tau}^{2} \tilde{\epsilon}_{\tau \tau},
\end{aligned}
$$

where $P_{0}\left(\nu_{\alpha} \rightarrow \nu_{\beta}\right)$ is the transition probability for $\nu_{\alpha} \rightarrow \nu_{\beta}$ without NSI matter effects, $\Delta_{31} \equiv \frac{\Delta m_{31}^{2}}{4 E}$, and $\Delta_{31}^{A} \equiv \frac{\Delta m_{31}^{2}-A}{4 E}$. Here, for the coefficient $\mathcal{C}_{\text {channel;element }}^{\text {order }}$ the upper index gives the order of this coefficient, and the lower one gives the channel and the element.

In Eq. (D1), the coefficients of $\epsilon_{\mu \tau}$ and $\tilde{\epsilon}_{\tau \tau}$ appear at leading order, i.e., at the order $\mathcal{C}_{\mu \rightarrow \mu \text {;element }}^{0}$ However, the coefficient of $\tilde{\epsilon}_{\tau \tau}$ is proportional to the factor $\left(c_{23}^{2}-s_{23}^{2}\right)$, which is suppressed since $\theta_{23} \sim 45^{\circ}$. The coefficients of $\tilde{\epsilon}_{e e}, \epsilon_{e \mu}$, and $\epsilon_{e \tau}$, which are of second, first, and first order, respectively, have less influence on $P\left(\nu_{\mu} \rightarrow \nu_{\mu}\right)$. Therefore, the impact of NSIs on the disappearance channel is dominated by $\epsilon_{\mu \tau}$. On the other hand, from Eq. (D2), it is obvious that the largest contributions to the transition probability are from $\epsilon_{e \mu}$ and $\epsilon_{e \tau}$, with coefficients of the first order. In Table XIII, we present the coefficients for $\alpha_{m n}$ based on Eqs. (D1) and (D2) and Table III.
TABLE XIII. The leading coefficient of each $\epsilon_{\alpha \beta}$ and $\alpha_{i j}$, for

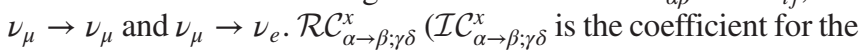
real (imaginary) part of $\gamma \delta$ as $\alpha \rightarrow \beta$, which is of the order $x$.

\begin{tabular}{lcc}
\hline \hline Channel & $\nu_{\mu} \rightarrow \nu_{\mu}$ & $\nu_{\mu} \rightarrow \nu_{e}$ \\
\hline$\tilde{\epsilon}_{e e}$ & $\mathcal{C}_{\mu \rightarrow \mu ; e e}^{2}$ & $\mathcal{C}_{\mu \rightarrow e ; e e}^{2}$ \\
$\tilde{\epsilon}_{\tau \tau}$ & $\mathcal{C}_{\mu \rightarrow \mu ; \tau \tau}^{0}$ & $\mathcal{C}_{\mu \rightarrow e ; \tau \tau}^{2}$ \\
$\epsilon_{e \mu}$ & $\mathcal{C}_{\mu \rightarrow \mu ; \mu \mu}^{1}$ & $\mathcal{C}_{\mu \rightarrow e ; \mu \mu}^{1}$ \\
$\epsilon_{e \tau}$ & $\mathcal{C}_{\mu \rightarrow \mu ; e \tau}^{1}$ & $\mathcal{C}_{\mu \rightarrow e ; \tau}^{1}$ \\
$\epsilon_{\mu \tau}$ & $\mathcal{C}_{\mu \rightarrow \mu ; \mu \tau}^{0}$ & $\mathcal{C}_{\mu \rightarrow e ; \mu \tau}^{2}$ \\
$\alpha_{12}$ & $\mathcal{C}_{\mu \rightarrow \mu ; e e}^{2}$ & $\mathcal{C}_{\mu \rightarrow e ; e e}^{2}$ \\
$\alpha_{13}$ & $-\sqrt{2} \mathcal{C}_{\mu \rightarrow \mu, \tau \tau}^{0}$ & $\frac{1}{2} \mathcal{C}_{\mu \rightarrow e ; e e}^{2}-\sqrt{2} \mathcal{C}_{\mu \rightarrow e ; \tau \tau}^{2}$ \\
$\alpha_{21}$ & $\frac{1}{\sqrt{6}} \mathcal{C}_{\mu \rightarrow \mu ; \mu \tau}^{0}$ & $\frac{1}{\sqrt{6}} \mathcal{R} \mathcal{C}_{\mu \rightarrow e ; e \mu}^{1}+\frac{1}{\sqrt{6}} \mathcal{R} \mathcal{C}_{\mu \rightarrow e ; e \tau}^{1}$ \\
$\alpha_{22}$ & $\frac{1}{\sqrt{3}} \mathcal{C}_{\mu \rightarrow \mu ; \mu \tau}^{0}$ & $\frac{1}{\sqrt{12}} \mathcal{R} \mathcal{C}_{\mu \rightarrow e ; e \mu}^{1}+\frac{1}{\sqrt{12}} \mathcal{R} \mathcal{C}_{\mu \rightarrow e ; e \tau}^{1}$ \\
$\alpha_{23}$ & $-\frac{1}{2} \mathcal{R} \mathcal{C}_{\mu \rightarrow \mu, e \mu}^{1}+\frac{1}{2} \mathcal{R} \mathcal{C}_{\mu \rightarrow \mu, e \tau}^{1}$ & $-\frac{1}{2} \mathcal{R} \mathcal{C}_{\mu \rightarrow e ; e \mu}^{1}+\frac{1}{2} \mathcal{R} \mathcal{C}_{\mu \rightarrow e ; e \tau}^{1}$ \\
$\alpha_{31}$ & $-\frac{1}{\sqrt{6}} \mathcal{I} \mathcal{C}_{\mu \rightarrow \mu, e \mu}^{1}+\frac{1}{\sqrt{6}} \mathcal{I} \mathcal{C}_{\mu \rightarrow \mu, e \tau}^{1}-\frac{1}{\sqrt{6}} \mathcal{I} \mathcal{C}_{\mu \rightarrow e ; e \mu}^{1}+\frac{1}{\sqrt{6}} \mathcal{I} \mathcal{C}_{\mu \rightarrow e ; e \tau}^{1}$ \\
$\alpha_{32}$ & $\frac{1}{\sqrt{12}} \mathcal{I} \mathcal{C}_{\mu \rightarrow \mu, e \mu}^{1}-\frac{1}{\sqrt{12}} \mathcal{I} \mathcal{C}_{\mu \rightarrow \mu, e \tau}^{1} \frac{1}{\sqrt{12}} \mathcal{I} \mathcal{C}_{\mu \rightarrow e ; e \mu}^{1}-\frac{1}{\sqrt{12}} \mathcal{I} \mathcal{C}_{\mu \rightarrow e ; e \tau}^{1}$ \\
$\alpha_{33}$ & $\frac{1}{2} \mathcal{I} \mathcal{C}_{\mu \rightarrow \mu, e \mu}^{1}+\frac{1}{2} \mathcal{I} \mathcal{C}_{\mu \rightarrow \mu, e \tau}^{1}$ & $\frac{1}{2} \mathcal{I} \mathcal{C}_{\mu \rightarrow e ; e \mu}^{1}+\frac{1}{2} \mathcal{I} \mathcal{C}_{\mu \rightarrow e ; e \tau}^{1}$ \\
\hline \hline
\end{tabular}


[1] Y. Fukuda et al. (Super-Kamiokande Collaboration), Phys. Rev. Lett. 81, 1562 (1998).

[2] S. Fukuda et al. (Super-Kamiokande Collaboration), Phys. Rev. Lett. 86, 5651 (2001); Q. R. Ahmad et al. (SNO Collaboration), Phys. Rev. Lett. 87, 071301 (2001); 89, 011301 (2002).

[3] M. H. Ahn et al. (K2K Collaboration), Phys. Rev. Lett. 90, 041801 (2003); K. Abe et al. (T2K Collaboration), Phys. Rev. Lett. 107, 041801 (2011).

[4] K. Eguchi et al. (KamLAND Collaboration), Phys. Rev. Lett. 90, 021802 (2003); F. P. An et al. (Daya Bay Collaboration), Phys. Rev. Lett. 108, 171803 (2012); J. K. Ahn et al. (RENO Collaboration), Phys. Rev. Lett. 108, 191802 (2012).

[5] R. Acciarri et al. (DUNE Collaboration), arXiv:1512.06148.

[6] K. Abe et al. (Hyper-Kamiokande Collaboration), Reports No. KEK-PREPRINT-2016-21 and No. ICRR-REPORT701-2016-1.

[7] F. An et al. (JUNO Collaboration), J. Phys. G 43, 030401 (2016).

[8] Z. Djurcic et al. (JUNO Collaboration), arXiv:1508.07166.

[9] M. Antonello et al. (MicroBooNE, LAr1-ND, and ICARUS-WA104 Collaborations), arXiv:1503.01520.

[10] D. Adey et al. (nuSTORM Collaboration), arXiv:1308.6822.

[11] J. Cao et al., Phys. Rev. ST Accel. Beams 17, 090101 (2014).

[12] S. Choubey et al. (IDS-NF Collaboration), arXiv:1112 .2853 .

[13] H. Minakata and S. J. Parke, Phys. Rev. D 87, 113005 (2013); S. Parke, Phys. Scripta T 158, 014013 (2013).

[14] M. Blennow, P. Coloma, P. Huber, and T. Schwetz, J. High Energy Phys. 03 (2014) 028; S. K. Agarwalla, Adv. High Energy Phys. 2014, 457803 (2014); P. Coloma, H. Minakata, and S. J. Parke, Phys. Rev. D 90, 093003 (2014); M. Blennow, P. Coloma, and E. FernandezMartinez, J. High Energy Phys. 03 (2015) 005; P. Ballett, S. F. King, S. Pascoli, N. W. Prouse, and T. Wang, Phys. Rev. D 96, 033003 (2017).

[15] T. Ohlsson, Rep. Prog. Phys. 76, 044201 (2013); O. G. Miranda and H. Nunokawa, New J. Phys. 17, 095002 (2015).

[16] S. Antusch, J. P. Baumann, and E. Fernandez-Martinez, Nucl. Phys. B810, 369 (2009).

[17] M. B. Gavela, D. Hernandez, T. Ota, and W. Winter, Phys. Rev. D 79, 013007 (2009).

[18] M. B. Wise and Y. Zhang, Phys. Rev. D 90, 053005 (2014).

[19] D. V. Forero and W. C. Huang, J. High Energy Phys. 03 (2017) 018.

[20] J. Heeck and W. Rodejohann, Phys. Rev. D 84, 075007 (2011).

[21] Y. Farzan, Phys. Lett. B 748, 311 (2015).

[22] D. Akimov et al. (COHERENT Collaboration), Science 1123, 357 (2017).

[23] M. C. Gonzalez-Garcia and M. Maltoni, J. High Energy Phys. 09 (2013) 152.

[24] M. Masud, A. Chatterjee, and P. Mehta, J. Phys. G 43, 095005 (2016); A. de Gouva and K. J. Kelly, Nucl. Phys. B908, 318 (2016); P. Coloma, J. High Energy Phys. 03 (2016) 016.
[25] J. Liao, D. Marfatia, and K. Whisnant, Phys. Rev. D 93, 093016 (2016); K. Huitu, T. J. Kärkkäinen, J. Maalampi, and S. Vihonen, Phys. Rev. D 93, 053016 (2016); P. Bakhti and Y. Farzan, J. High Energy Phys. 07 (2016) 109; M. Masud and P. Mehta, Phys. Rev. D 94, 013014 (2016); P. Coloma and T. Schwetz, Phys. Rev. D 94, 055005 (2016); 95, 079903(E) (2017); M. Masud and P. Mehta, Phys. Rev. D 94, 053007 (2016); M. Blennow, S. Choubey, T. Ohlsson, D. Pramanik, and S. K. Raut, J. High Energy Phys. 08 (2016) 090.

[26] S. K. Agarwalla, S. S. Chatterjee, and A. Palazzo, Phys. Lett. B 762, 64 (2016); S. F. Ge and A. Y. Smirnov, J. High Energy Phys. 10 (2016) 138; S. Fukasawa, M. Ghosh, and O. Yasuda, Phys. Rev. D 95, 055005 (2017); K. N. Deepthi, S. Goswami, and N. Nath, Phys. Rev. D 96, 075023 (2017); J. Liao, D. Marfatia, and K. Whisnant, J. High Energy Phys. 01 (2017) 071.

[27] P. Coloma, P. B. Denton, M. C. Gonzalez-Garcia, M. Maltoni, and T. Schwetz, J. High Energy Phys. 04 (2017) 116; M. Masud, M. Bishai, and P. Mehta, Sci. Rep. 9, 352 (2019); K. N. Deepthi, S. Goswami, and N. Nath, Nucl. Phys. B936, 91 (2018).

[28] M. Blennow, P. Coloma, E. Fernandez-Martinez, J. Hernandez-Garcia, and J. Lopez-Pavon, J. High Energy Phys. 04 (2017) 153.

[29] N. C. Ribeiro, H. Minakata, H. Nunokawa, S. Uchinami, and R. Zukanovich-Funchal, J. High Energy Phys. 12 (2007) 002; P. Coloma, A. Donini, J. Lopez-Pavon, and H. Minakata, J. High Energy Phys. 08 (2011) 036.

[30] J. Tang and Y. Zhang, Phys. Rev. D 97, 035018 (2018).

[31] Z. Rahman, A. Dasgupta, and R. Adhikari, J. Phys. G 42, 065001 (2015).

[32] A. Dasgupta, Z. Rahman, and R. Adhikari, arXiv: 1210.4801.

[33] P. Coloma, A. Donini, J. Lopez-Pavon, and H. Minakata, J. High Energy Phys. 08 (2011) 036.

[34] E. Ma and G. Rajasekaran, Phys. Rev. D 64, 113012 (2001).

[35] G. Altarelli and F. Feruglio, Nucl. Phys. B720, 64 (2005).

[36] G. Altarelli and F. Feruglio, Nucl. Phys. B741, 215 (2006).

[37] C. S. Lam, Phys. Rev. D 78, 073015 (2008).

[38] P. F. Harrison, D. H. Perkins, and W. G. Scott, Phys. Lett. B 530, 167 (2002); Z. Z. Xing, Phys. Lett. B 533, 85 (2002); P. F. Harrison and W. G. Scott, Phys. Lett. B 535, 163 (2002); X. G. He and A. Zee, Phys. Lett. B 560, 87 (2003).

[39] For some reviews, see G. Altarelli and F. Feruglio, Rev. Mod. Phys. 82, 2701 (2010); S. F. King and C. Luhn, Rep. Prog. Phys. 76, 056201 (2013); S. F. King, A. Merle, S. Morisi, Y. Shimizu, and M. Tanimoto, New J. Phys. 16, 045018 (2014).

[40] E. Ma, Phys. Rev. D 82, 037301 (2010).

[41] R. de Adelhart Toorop, F. Bazzocchi, L. Merlo, and A. Paris, J. High Energy Phys. 03 (2011) 035; 03 (2011) 040.

[42] A. Degee, I. P. Ivanov, and V. Keus, J. High Energy Phys. 02 (2013) 125; V. Keus, S. F. King, and S. Moretti, J. High Energy Phys. 01 (2014) 052.

[43] J. Heeck, M. Holthausen, W. Rodejohann, and Y. Shimizu, Nucl. Phys. B896, 281 (2015); I. de Medeiros Varzielas, O. Fischer, and V. Maurer, J. High Energy Phys. 08 (2015) 080.

[44] T. Kobayashi, Y. Omura, F. Takayama, and D. Yasuhara, J. High Energy Phys. 10 (2015) 042. 
[45] S. Pascoli and Y. L. Zhou, J. High Energy Phys. 10 (2016) 145.

[46] Y. Muramatsu, T. Nomura, Y. Shimizu, and H. Yokoya, Phys. Rev. D 97, 015003 (2018).

[47] Y. Farzan and I. M. Shoemaker, J. High Energy Phys. 07 (2016) 033.

[48] Y. Farzan and J. Heeck, Phys. Rev. D 94, 053010 (2016).

[49] K. S. Babu, A. Friedland, P. A. N. Machado, and I. Mocioiu, J. High Energy Phys. 12 (2017) 096.

[50] S. Pascoli and Y. L. Zhou, J. High Energy Phys. 06 (2016) 073.

[51] I. Girardi, S. T. Petcov, A. J. Stuart, and A. V. Titov, Nucl. Phys. B902, 1 (2016); S. F. King, J. Phys. G 42, 123001 (2015); C. C. Li, J. N. Lu, and G. J. Ding, Nucl. Phys. B913, 110 (2016); S. K. Kang, Y. Shimizu, K. Takagi, S. Takahashi, and M. Tanimoto, Prog. Theor. Exp. Phys. (2018), 083B01.

[52] E. Fernandez-Martinez, J. Hernandez-Garcia, and J. Lopez-Pavon, J. High Energy Phys. 08 (2016) 033.

[53] S. Antusch and O. Fischer, J. High Energy Phys. 10 (2014) 094.

[54] K. Hayasaka et al., Phys. Lett. B 687, 139 (2010).

[55] B. Aubert et al. (BABAR Collaboration), Phys. Rev. Lett. 104, 021802 (2010).

[56] A. M. Baldini et al. (MEG Collaboration), Eur. Phys. J. C 76, 434 (2016).
[57] D. Wyler and L. Wolfenstein, Nucl. Phys. B218, 205 (1983); R. N. Mohapatra, Phys. Rev. Lett. 56, 561 (1986); R. N. Mohapatra and J. W. F. Valle, Phys. Rev. D 34, 1642 (1986).

[58] R. N. Mohapatra, M. K. Parida, and G. Rajasekaran, Phys. Rev. D 69, 053007 (2004).

[59] C. Biggio, M. Blennow, and E. Fernandez-Martinez, J. High Energy Phys. 03 (2009) 139.

[60] C. Biggio, M. Blennow, and E. Fernandez-Martinez, J. High Energy Phys. 08 (2009) 090.

[61] Q. H. Cao, G. Li, K. P. Xie, and J. Zhang, Phys. Rev. D 97, 115036 (2018).

[62] I. Esteban, M. C. Gonzalez-Garcia, M. Maltoni, I. MartinezSoler, and T. Schwetz, J. High Energy Phys. 01 (2017) 087.

[63] S. S. Wilks, Ann. Math. Stat. 9, 60 (1938).

[64] P. Huber, M. Lindner, and W. Winter, Comput. Phys. Commun. 167, 195 (2005).

[65] P. Huber, J. Kopp, M. Lindner, M. Rolinec, and W. Winter, Comput. Phys. Commun. 177, 432 (2007).

[66] T. Alion et al. (DUNE Collaboration), arXiv:1606.09550.

[67] T. Kikuchi, H. Minakata, and S. Uchinami, J. High Energy Phys. 03 (2009) 114. 